\title{
Seiberg-Witten-Floer Homology and Gluing Formulae
}

\author{
Alan L. Carey, Bai-Ling Wang
}

\begin{abstract}
This paper gives a detailed construction of Seiberg-Witten-Floer homology for a closed oriented 3-manifold with a non-torsion $\operatorname{Spin}^{c}$ structure. Gluing formulae for certain 4-dimensional manifolds splitting along an embedded 3-manifold are obtained.
\end{abstract}

\section{Contents}

\begin{tabular}{lll}
\hline 1 & Introduction and statement of results & 2
\end{tabular}

\begin{tabular}{|lll}
\hline 2 & Seiberg-Witten monopoles on 3-manifolds: review & 7
\end{tabular}

$\begin{array}{lll}3 & \text { Seiberg-Witten-Floer homology } & 12\end{array}$

3.1 The basics of the CSD functional: critical points, relative indices . . . . . . . . 12

3.2 Estimates on gradient flowlines $\ldots \ldots \ldots \ldots \ldots \ldots \ldots$

3.3 Moduli spaces of flowlines: transversality, compactification and orientibility. . . . 22

3.4 Seiberg-Witten-Floer homology: definition and properties . . . . . . . . . . . . 35

3.5 Variants of the Seiberg-Witten-Floer homology . . . . . . . . . . . . . . . . . . . 40

4 Gluing formulae for 4-dimensional Seiberg-Witten invariants

4.1 Moduli space for 4-manifolds with cylindrical ends . . . . . . . . . . . . . . . . 44

4.2 Relative invariants for 4-manifolds with boundary . . . . . . . . . . . . . . 50

4.3 Gluing formulae for the Seiberg-Witten invariants. . . . . . . . . . . . . . . . 53

\begin{tabular}{ll}
\hline References & 60
\end{tabular} 


\section{Introduction and statement of results}

In this paper we give a detailed account of the construction of Seiberg-Witten-Floer homology for a closed oriented 3-manifold $Y$ with a non-torsion $\operatorname{Spin}^{c}$ structure $\mathfrak{s}$ and discuss some applications. The basic idea is to think of the Seiberg-Witten equations as defining critical points for the Chern-Simons-Dirac functional. This functional is on the configuration space (which consists of pairs of $U(1)$ connections and spinors modulo the gauge transformations) but may have degenerate critical points. These can be removed by a suitable perturbation. To construct the Seiberg-Witten-Floer homology one proceeds to study the time independent gradient flow lines from one critical point to another. These flow lines or trajectories may be thought of as solutions to the Seiberg-Witten equations in temporal gauge on the four manifold $Y \times \mathbb{R}$. A suitable choice of perturbation of the Chern-Simons-Dirac functional gives the desired properties (such as smoothness and transversality) for the moduli space of trajectory flowlines.

We now summarise the contents of the paper. In section 2, we review the basic properties of Seiberg-Witten monopoles on a closed, oriented 3-manifold $(Y, g, \mathfrak{s})$ with $\operatorname{Spin}^{c}$ structure $\mathfrak{s}$ and metric $g$. Section 3 gives a complete account of the infinite dimensional Morse theory for the Chern-Simons-Dirac functional whose critical points satisfy the Seiberg-Witten equations on $(Y, g, \mathfrak{s})$. The downward gradient flowline of the Chern-Simons-Dirac functional, thought as a solution to the Seiberg-Witten equations on a cylinder $\mathbb{R} \times Y$, is endowed with an energy function (22), such that when the energy is sufficiently small, the flowline stays in a small neighbourhood of some critical point (see Lemma 3.8). This enables us to analyse the local behaviour of any gradient flowline near a critical point in Proposition 3.9, under the small energy condition.

In order to achieve transversality for the moduli space of flowline, we adopt the sort of perturbations suggested by Kronheimer [14] with an additional condition 3.12. We also show that, for generic perturbations, the moduli space of flowlines with finite energy is smooth for each connected component (cf. Theorem 3.16), which can be compactified to a smooth manifold with corners (cf. Proposition 3.18).

The analysis developed in this paper can be applied to more general cases but we only discuss one namely Seiberg-Witten-Floer homology theory for a closed oriented 3-manifold $Y$ with $b_{1}(Y)>0$ and a $\operatorname{Spin}^{c}$ structure $\mathfrak{s}$ such that $c_{1}(\mathfrak{s})$ is non-torsion. For any rational homology 3sphere, there exists an equivariant Seiberg-Witten Floer homology in [20], which is a topological invariant of the underlying manifold and $\operatorname{Spin}^{c}$ structure. For the case of $(Y, \mathfrak{s})$ with $b_{1}(Y)>0$ and $c_{1}(\mathfrak{s})=0$, Seiberg-Witten-Floer homology theory will be investigated elsewhere [21]. The results on Seiberg-Witten-Floer homology for a closed 3-manifold $Y$ with $b_{1}(Y)>0$ and a nontorsion $\mathrm{Spin}^{c}$ structure in this paper are summarized in the following theorem, whose proof will be given in section 3 . 
Theorem 1.1. For any closed oriented 3-manifold $Y$ with $b_{1}(Y)>0$ and a $\operatorname{Spin}^{c}$ structure $\mathfrak{s}$ such that $c_{1}(\mathfrak{s}) \neq 0$ in $H^{2}(Y, \mathbb{Z}) /$ Torsion, let $d(\mathfrak{s})$ be the divisibility of $c_{1}(\mathfrak{s})$ in $H^{2}(Y, \mathbb{Z}) /$ Torsion, that is

$$
d(\mathfrak{s})=\text { g.c.d. }\left\{\left\langle c_{1}(\mathfrak{s}), \sigma\right\rangle: \text { for } \sigma \in H_{2}(Y, \mathbb{Z})\right\} .
$$

Then there exists a finitely generated Seiberg-Witten-Floer complex whose homology $H F_{*}^{S W}(Y, \mathfrak{s})$ satisfies the following properties:

1. $H F_{*}^{S W}(Y, \mathfrak{s})$ is a topological invariant of $(Y, \mathfrak{s})$ and is a $\mathbb{Z}_{d(\mathfrak{s})}$-graded Abelian group.

2. There is an action of

$$
\mathbb{A}(Y)=\operatorname{Sym}^{*}\left(H_{0}(Y, \mathbb{Z})\right) \otimes \Lambda^{*}\left(H_{1}(Y, \mathbb{Z}) / \text { Torsion }\right)
$$

on $H F_{*}^{S W}(Y, \mathfrak{s})$ with elements in $H_{0}(Y, \mathbb{Z})$ and $H_{1}(Y, \mathbb{Z}) /$ Torsion decreasing degree in $H F_{*}^{S W}(Y, \mathfrak{s})$ by 2 and 1 respectively.

3. For $(-Y,-\mathfrak{s})$, where $-Y$ is $Y$ with the reversed orientation and $-\mathfrak{s}$ is the induced $\operatorname{Spin}^{c}$ structure, the corresponding Seiberg-Witten-Floer complex $C_{*}(-Y,-\mathfrak{s})$ is the dual complex of $C_{*}(Y, \mathfrak{s})$. There is a natural pairing

$$
\langle,\rangle: \quad H F_{*}^{S W}(Y, \mathfrak{s}) \times H F_{-*}^{S W}(-Y,-\mathfrak{s}) \longrightarrow \mathbb{Z}
$$

such that $<z . \Xi_{1}, \Xi_{2}>=<\Xi_{1}, z . \Xi_{2}>$ for any $z \in \mathbb{A}(Y) \cong \mathbb{A}(-Y)$ and any cycles $\Xi_{1} \in H F_{*}^{S W}(Y, \mathfrak{s})$ and $\Xi_{2} \in H F_{-*}^{S W}(-Y,-\mathfrak{s})$ respectively.

4. For any subgroup $K \subseteq \operatorname{Ker}\left(c_{1}(\mathfrak{s})\right) \subset H^{1}(Y, \mathbb{Z})$, there is a variant of Seiberg-WittenFloer homology denoted by $H_{*,[K]}^{S W}(Y, \mathfrak{s}) . H F_{*,[K]}^{S W}(Y, \mathfrak{s})$ is a topological invariant and a $\mathbb{Z}$-graded $\mathbb{A}(Y)$ module. For any element $[u] \in H^{1}(Y, \mathbb{Z}) / K$, there is an action of $[u]$ on $H F_{*,[K]}^{S W}(Y, \mathfrak{s})$ which decreases degree by $<[u] \wedge c_{1}(\mathfrak{s}),[Y]>$. There exists a $\mathbb{A}(Y)$ equivariant homomorphism:

$$
\pi: \quad H F_{*,[K]}^{S W}(Y, \mathfrak{s}) \longrightarrow H F_{*}^{S W}(Y, \mathfrak{s}) .
$$

There is also a natural pairing

$$
\langle,\rangle: \quad H F_{*,[K]}^{S W}(Y, \mathfrak{s}) \times H F_{-*,[K]}^{S W}(-Y,-\mathfrak{s}) \longrightarrow \mathbb{Z}
$$

satisfying $<z \cdot \Xi_{1}, \Xi_{2}>=<\Xi_{1}, z \cdot \Xi_{2}>$ for any $z \in \mathbb{A}(Y) \cong \mathbb{A}(-Y)$ and any cycles $\Xi_{1} \in H F_{*,[K]}^{S W}(Y, \mathfrak{s})$ and $\Xi_{2} \in H F_{-*,[K]}^{S W}(-Y,-\mathfrak{s})$ respectively. If $K_{1} \subset K_{2}$ are two subgroups in $\operatorname{Ker}\left(c_{1}(\mathfrak{s})\right)$, there is a $\mathbb{A}(Y)$-equivariant homomorphism:

$$
H F_{*,\left[K_{1}\right]}^{S W}(Y, \mathfrak{s}) \longrightarrow H F_{*,\left[K_{2}\right]}^{S W}(Y, \mathfrak{s}) .
$$


Moreover, $H F_{*,\left[K \operatorname{Ker}\left(c_{1}(\mathfrak{s})\right)\right]}^{S W, \mathfrak{s})}$ satisfies the following periodicity property:

$$
H F_{m,\left[\operatorname{Ker}\left(c_{1}(\mathfrak{s})\right)\right]}^{S W}(Y, \mathfrak{s}) \cong H F_{m(\bmod d(\mathfrak{s}))}^{S W}(Y, \mathfrak{s}),
$$

for any $m \in \mathbb{Z}$.

Section 4 gives an application to the problem of associating invariants to a 4-manifold with boundary and gluing formulae for 4-dimensional monopole invariants. The setup here is to start with a 4-manifold $\left(X_{+}, \mathfrak{s}_{+}\right)$with cylindrical end modelled on $(Y, \mathfrak{t})$ : that is, over the end $[-2, \infty) \times Y$, there is a fixed isomorphism between the restrction of $\mathfrak{s}_{+}$and the pull-back Spin ${ }^{c}$ structure of $\mathfrak{t}$. In addition, we assume $c_{1}(\operatorname{det}(\mathfrak{t}))$ is non-torsion.

Finite energy solutions to the Seiberg-Witten equations on $X_{+}$define a moduli space with finite variations of the perturbed Chern-Simons-Dirac functional on the end. We associate to this moduli space a boundary asymptotic value map

$$
\partial_{\infty}: \quad \mathcal{M}_{X_{+}}\left(\mathfrak{s}_{+}\right) \longrightarrow \mathcal{M}_{Y, X_{+}}(\mathfrak{t}, \eta)
$$

where $\mathcal{M}_{Y, X_{+}}(\mathfrak{t}, \eta)$ is the quotient of solutions to the perturbed Seiberg-Witten equations on $(Y, \mathfrak{t}, \eta)$ by the action of those gauge transformations which can be extended to $X_{+}$. In fact,

$$
\pi_{+}: \quad \mathcal{M}_{Y, X_{+}}(\mathfrak{t}, \eta) \longrightarrow \mathcal{M}_{Y}(\mathfrak{t}, \eta)
$$

is a covering map with fiber an $H^{1}(Y, \mathbb{Z}) / \operatorname{Im}\left(i_{+}^{*}\right)$-homogeneous space. Here $\operatorname{Im}\left(i_{+}^{*}\right)$ is the range of the map $i_{+}^{*}: H^{1}\left(X_{+}, \mathbb{Z}\right) \rightarrow H^{1}(Y, \mathbb{Z})$.

Over the end $[-2, \infty) \times Y$, if $\left(X_{+}, \mathfrak{s}_{+}\right)$is modelled on $(Y, \mathfrak{t})$ up to an isomorphism $u \in C^{\infty}(Y, U(1))$, then the corresponding asymptotic value map is given by $[u] \circ \partial_{\infty}$ with $[u] \in H^{1}(Y, \mathbb{Z}) / \operatorname{Im}\left(i_{+}^{*}\right)$ determined by the connected component of $C^{\infty}(Y, U(1))$ which $u$ belongs to.

Fix an orientations on $\Lambda^{\text {top }} H^{1}\left(X_{+}(0), Y ; \mathbb{R}\right) \otimes \Lambda^{t o p} H^{2,+}\left(X_{+}(0), Y ; \mathbb{R}\right)$. The structure of the fiber for $\partial_{\infty}$ and its compactification are discussed in Proposition 4.2 and Proposition 4.4. These propositions tell us that for each $\Gamma_{\alpha} \in \mathcal{M}_{Y, X_{+}}(\mathfrak{t}, \eta)$, there is a Baire set of self-dual 2-forms with compact support such that the fiber of $\partial_{\infty}$ (if non-empty), denoted by $\mathcal{M}_{X_{+}}\left(\mathfrak{s}_{+}, \Gamma_{\alpha}\right)$, is an oriented, smooth manifold of dimension $\mathfrak{i}_{X_{+}}\left(\Gamma_{\alpha}\right)$ given by (54). It can be compactified to a smooth manifold with corners. In particular, for any $d \geq 0$, the components of dimension $d$ in $\mathcal{M}_{X_{+}}\left(\mathfrak{s}_{+}\right)$, denoted by $\mathcal{M}_{X_{+}}^{d}\left(\mathfrak{s}_{+}\right)$, can be described as

$$
\mathcal{M}_{X_{+}}^{d}\left(\mathfrak{s}_{+}\right)=\cup_{\alpha \in \mathcal{M}_{Y}(\mathfrak{t}, \eta)}\left(\bigcup_{\Gamma_{\alpha} \in \pi_{+}^{-1}(\alpha), \mathfrak{i}_{X_{+}}\left(\Gamma_{\alpha}\right)=d} \mathcal{M}_{X_{+}}\left(\mathfrak{s}_{+}, \Gamma_{\alpha}\right)\right) .
$$

Here $\mathcal{M}_{X_{+}}\left(\mathfrak{s}_{+}, \Gamma_{\alpha}\right)=\partial_{\infty}^{-1}\left(\Gamma_{\alpha}\right)$. 
We then define a relative Seiberg-Witten invariant of $X_{+}$. These relative Seiberg-Witten invariants take values in the homology groups $H F_{*,\left[\operatorname{Im}\left(i_{+}^{*}\right)\right]}^{S W}(Y, \mathfrak{t})$. We denote this invariant of $X_{+}$by $S W_{X_{+}}\left(\mathfrak{s}_{+}\right)$, which is a linear functional

$$
S W_{X_{+}}\left(\mathfrak{s}_{+}, \cdot\right): \quad \mathbb{A}\left(X_{+}\right) \longrightarrow H F_{*,\left[\operatorname{Im}\left(i_{+}^{*}\right)\right]}^{S W}(Y, \mathfrak{t}),
$$

where $\mathbb{A}\left(X_{+}\right)=\operatorname{Sym}^{*}\left(H_{0}\left(X_{+}, \mathbb{Z}\right)\right) \otimes \Lambda^{*}\left(H_{1}\left(X_{1}, \mathbb{Z}\right) /\right.$ Torsion $)$ the free graded algebra generated by the class of elements in $H_{0}\left(X_{+}, \mathbb{Z}\right)$ and $H_{1}\left(X_{+}, \mathbb{Z}\right)$ with degree 2 and 1 respectively.

$S W_{X_{+}}\left(\mathfrak{s}_{+}, z\right)$ with $\operatorname{deg}(z)=d$ can be represented in terms of the Seiberg-Witten invariant for the moduli space $\mathcal{M}_{X_{+}}^{d}\left(\mathfrak{s}_{+}\right)$in (4), that is, for each non-empty component $\mathcal{M}_{X_{+}}\left(\mathfrak{s}_{+}, \Gamma_{\alpha}\right)$ with $\mathfrak{i}_{X_{+}}\left(\Gamma_{\alpha}\right)=d$, there is a topological invariant $S W_{X_{+}}\left(\mathfrak{s}_{+}, z, \Gamma_{\alpha}\right)$ defined in subsection 4.2 , with

$$
S W_{X_{+}}\left(\mathfrak{s}_{+}, z\right)=\sum_{\alpha \in \mathcal{M}_{Y}(\mathfrak{t}, \eta)} \sum_{\Gamma_{\alpha} \in \pi_{+}^{-1}(\alpha)} S W_{X_{+}}\left(\mathfrak{s}_{+}, z, \Gamma_{\alpha}\right)<\Gamma_{\alpha}>
$$

Section 4 also describes results on closed smooth 4-manifolds $(X, \mathfrak{s})$ with $\operatorname{Spin}^{c}$ structure $\mathfrak{s}$ and a smooth embedded separating 3-manifold $Y$ such that there is an embedding $[-2,2] \times Y$ in $X$ and $\mathfrak{t}=\left.\mathfrak{s}\right|_{Y}$ is a non-torsion $\operatorname{Spin}^{c}$ structure on $Y$. Consider a 1-parameter family of metrics $\left\{g_{R}\right\}_{R>0}$ on $X$ such that for each $X(R)=\left(X, g_{R}\right)$, there are an isometrically embedded submanifold $\left([-R-2, R+2] \times Y, d t^{2}+g_{Y}\right)$ and two 4-manifolds $X_{ \pm}(R)$ obtained by setting $X(R)=X_{+}(R) \cup X_{-}(R)$. As $R \rightarrow \infty, X(R)$ has a geometric limit, two 4-manifolds with cylindrical ends, denoted by $X_{ \pm}(\infty)$. Note that there are induced $\operatorname{Spin}^{c}$ structures $\mathfrak{s}_{ \pm}$on $X_{ \pm}(\infty)$ from the $\operatorname{Spin}^{c}$ structure $\mathfrak{s}$ on $X$.

Denote by $i_{ \pm}$the boundary embedding maps of $Y$ in $X_{ \pm}(0)$, and by $\operatorname{Im}\left(i_{ \pm}^{*}\right)$ the ranges of the maps $H^{1}\left(X_{ \pm}(0), \mathbb{Z}\right) \rightarrow H^{1}(Y, \mathbb{Z})$. Then we have the following commutative diagram relating various subgroups $\operatorname{Im}\left(i_{+}^{*}\right) \cap \operatorname{Im}\left(i_{-}^{*}\right), \operatorname{Im}\left(i_{ \pm}^{*}\right)$ and $\operatorname{Im}\left(i_{+}^{*}\right)+\operatorname{Im}\left(i_{-}^{*}\right)$ of $\operatorname{Ker}\left(c_{1}(\mathfrak{t})\right)$ in $H^{1}(Y, Z)$ :

$$
\frac{H^{1}(Y, Z)}{\operatorname{Im}\left(i_{+}^{*}\right) \cap \operatorname{Im}\left(i_{-}^{*}\right)} \nearrow_{\frac{H^{1}(Y, Z)}{\operatorname{Im}\left(i_{-}^{*}\right)}} \nearrow^{\frac{H^{1}(Y, Z)}{\operatorname{Im}\left(i_{+}^{*}\right)}} \searrow \frac{H^{1}(Y, Z)}{\operatorname{Im}\left(i_{+}^{*}\right)+\operatorname{Im}\left(i_{-}^{*}\right)}
$$

which induces a commutative diagram of $\mathbb{A}(Y)$-equivariant homomorphisms between various variants of the Seiberg-Witten-Floer homologies for $(Y, \mathfrak{t})$ :

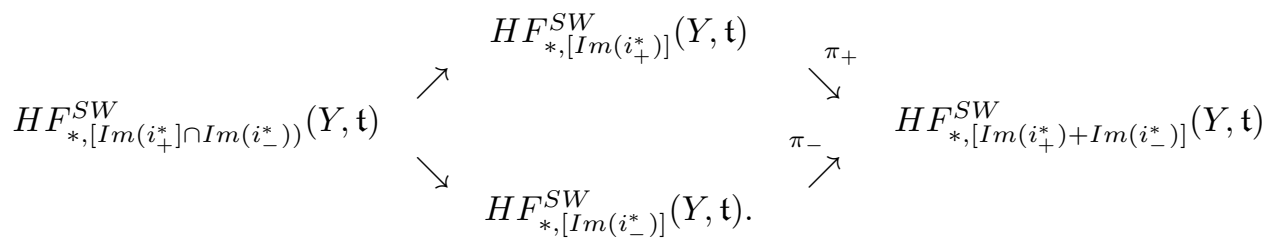


With these notations understood, the relative invariants for $\left(X_{ \pm}(\infty), \mathfrak{s}_{ \pm}\right)$are linear functionals

$$
S W_{X_{ \pm}}\left(\mathfrak{s}_{ \pm}, \cdot\right): \quad \mathbb{A}\left(X_{ \pm}\right) \longrightarrow H F_{*,\left[I m\left(i_{ \pm}^{*}\right)\right]}^{S W}( \pm Y, \pm \mathfrak{t})
$$

Fix an orientation on the line $\Lambda^{t o p} H^{1}(X, \mathbb{R}) \otimes \Lambda^{t o p} H^{2,+}(X, \mathbb{R})$ which is induced from tensoring the orientations on $\Lambda^{\text {top }} H^{1}\left(X_{+}(0), Y ; \mathbb{R}\right) \otimes \Lambda^{t o p} H^{2,+}\left(X_{+}(0), Y ; \mathbb{R}\right)$ and $\Lambda^{t o p} H^{1}\left(X_{-}(0), Y ; \mathbb{R}\right) \otimes \Lambda^{t o p} H^{2,+}\left(X_{-}(0), Y ; \mathbb{R}\right)$. When $b_{2}^{+}=1$, we also need to fix an orientation on the line $\Lambda^{\text {top }} H^{2,+}(X, \mathbb{R})$.

The Seiberg-Witten invariant for a closed manifold $(X, \mathfrak{s})$ is a linear functional

$$
S W_{X}(\mathfrak{s}, \cdot): \mathbb{A}(X) \mapsto \mathbb{Z}
$$

as defined in 34] where $\mathbb{A}(X)=\operatorname{Sym}^{*}\left(H_{0}(X)\right) \otimes \Lambda^{*}\left(H_{1}(X) /\right.$ Torsion $)$. Note that there is indeterminacy in the $\operatorname{Spin}^{c}$ structures on $X(R)$ obtained by gluing $\mathfrak{s}_{ \pm}$along $(Y, \mathfrak{t})$. The set of these $\operatorname{Spin}^{c}$ structures, say $\operatorname{Spin}^{c}\left(X, \mathfrak{s}_{ \pm}\right)$, can be represented by

$$
\operatorname{Spin}^{c}\left(X, \mathfrak{s}_{ \pm}\right)=\left\{\mathfrak{s}_{+} \#_{[u]} \mathfrak{s}_{-} \mid[u] \in \frac{H^{1}(Y, Z)}{\operatorname{Im}\left(i_{+}^{*}\right)+\operatorname{Im}\left(i_{-}^{*}\right)}\right\} .
$$

Here $\mathfrak{s}_{+} \#_{[u]^{\mathfrak{s}_{-}}}$is the resulting $\operatorname{Spin}^{c}$ structure on $X$ obtained by gluing $\mathfrak{s}_{ \pm}$using the gauge transformation $u$ representing $[u]$.

Now our main gluing formulae along $(Y, \mathfrak{t})$ with $b_{1}(Y)>0$ and $c_{1}(\mathfrak{t}) \neq 0$ are contained in the following theorem.

Theorem 1.2. Let $X$ be a closed 4-manifold with $b_{2}^{+} \geq 1$ which can be split as $X=X_{+} \cup_{Y} X_{-}$, where $X_{+}$and $X_{-}$are 4 -manifolds with boundary $\partial X_{+}=-\partial X_{-}=Y$. Suppose that we have $\operatorname{Spin}^{c}$ structures $\mathfrak{s}_{+}$and $\mathfrak{s}_{-}$on $X_{+}$and $X_{-}$respectively which restrict to $\mathfrak{t}$ on the boundary. Assume that $b_{1}(Y)>0$ and $c_{1}(\mathfrak{t})$ is non-torsion then the Seiberg-Witten invariants for $\left(X, \mathfrak{s}_{+} \#_{[u]^{\mathfrak{s}_{-}}}\right)$can be expressed as

$$
S W_{X}\left(\mathfrak{s}_{+} \#_{[u]} \mathfrak{s}_{-}, z_{+} z_{-}\right)=\left\langle[u]\left(\pi_{+}\left(S W_{X_{+}}\left(\mathfrak{s}_{+}, z_{+}\right)\right)\right), \pi_{-}\left(S W_{X_{-}}\left(\mathfrak{s}_{-}, z_{-}\right)\right)\right\rangle
$$

where $[u]$ acts on $H F_{*,\left[\operatorname{Im}\left(i_{+}^{*}\right)+\operatorname{Im}\left(i_{-}^{*}\right)\right]}^{S W}(Y, \mathfrak{t}), z_{ \pm} \in \mathbb{A}\left(X_{ \pm}\right), \pi_{ \pm}$are the homomorphisms given in (1), and the pairing on the right hand side is the natural pairing on

$$
H F_{*,\left[\operatorname{Im}\left(i_{+}^{*}\right)+\operatorname{Im}\left(i_{-}^{*}\right)\right]}^{S W}(Y, \mathfrak{t}) \times H F_{*,\left[\operatorname{Im}\left(i_{+}^{*}\right)+\operatorname{Im}\left(i_{-}^{*}\right)\right]}^{S W}(-Y,-\mathfrak{t})
$$

with the degrees in $H F_{*,\left[\operatorname{Im}\left(i_{+}^{*}\right)+\operatorname{Im}\left(i_{-}^{*}\right)\right]}(-Y,-\mathfrak{t})$ shifted by

$$
d_{X}(\mathfrak{s})=\frac{1}{4}\left(c_{1}(\mathfrak{s})^{2}-(2 \chi(X)+3 \sigma(X))\right)=\operatorname{deg}\left(z_{1}\right)+\operatorname{deg}\left(z_{2}\right) .
$$


When $b_{2}^{+}=1$, the Seiberg-Witten invariants $S W_{X}(\mathfrak{s}, \cdot)$ are defined with a fixed orientation on $H^{2,+}(X, \mathbb{R})$ such that $c_{1}(\mathfrak{s}) \cdot \omega^{+}>0$ for an oriented generator $\omega^{+}$of $H^{2,+}(X, \mathbb{R})$. In particular, there is a set $\mathcal{S}$ of $\operatorname{Spin}^{c}$ structures $\mathfrak{s} \in \operatorname{Spin}^{c}\left(X, \mathfrak{s}_{ \pm}\right)$with $d_{X}(\mathfrak{s})=\operatorname{deg}\left(z_{1}\right)+\operatorname{deg}\left(z_{2}\right)$ such that

$$
\sum_{\mathfrak{s} \in \mathcal{S}} S W_{X}\left(\mathfrak{s}, z_{+} z_{-}\right)=\left\langle\pi\left(S W_{X_{+}}\left(\mathfrak{s}_{+}, z_{+}\right)\right), \pi\left(S W_{X_{-}}\left(\mathfrak{s}_{-}, z_{-}\right)\right)\right\rangle
$$

Here $\pi\left(S W_{X_{ \pm}}\left(\mathfrak{s}_{ \pm}, z_{ \pm}\right)\right)$are elements in $H F_{*}^{S W}( \pm Y, \pm \mathfrak{t})$ under the homomorphism (1) and the pairing on the right hand side is the natural pairing on $H F_{*}^{S W}(Y, \mathfrak{t}) \times H F_{*}^{S W}(-Y,-\mathfrak{t}) \rightarrow \mathbb{Z}$.

This gluing theorem generalizes the gluing result of Morgan-Szabo-Taubes (Theorem 9.5 in [25]), has already found applications to the study of Seiberg-Witten-Floer homology as in [26]. We like to thank Vicente Munoz for his help in providing examples to test our gluing formulae.

We would like to acknowledge our gratitude to Matilde Marcolli, Tom Mrowka and Cliff Taubes for many useful correspondences. The authors also thank the Max-Planck-Institut fur Mathematik in Bonn for hospitality and support. This research was supported in part by the Australian Research Council.

\section{Seiberg-Witten monopoles on 3-manifolds: review}

Let $Y$ be a closed, oriented 3-manifold equipped with a Riemannian metric $g$. To introduce the Seiberg-Witten equations on $(Y, g)$, we need to choose a $\operatorname{Spin}^{c}$ structure on $Y$. This is a lift of the $S O(3)$-frame bundle on $Y$ to a $\operatorname{Spin}^{c}(3)$-bundle $P$. Note that $\operatorname{Spin}^{c}(3)=U(2)$, the determinant homomorphism $\operatorname{Spin}^{c}(3) \rightarrow U(1)$ determines a principal $U(1)$-bundle, the corresponding complex line bundle is called the determinant line bundle of the $\operatorname{Spin}^{c}$ structure $P$.

The isomorphism classes of $\operatorname{Spin}^{c}$ structures are classified by the first Chern class of the determinant line bundle. Given a $\operatorname{Spin}^{c}$ structure $P$, there is an associated $\operatorname{Spin}^{c}$ bundle

$$
W=P \times_{U(2)} \mathbb{C}^{2}
$$

which is complex vector bundle whose sections form a module for the Clifford bundle on $(Y, g)$. As a vector space the Clifford bundle can be identified with the exterior bundle on $T^{*} Y$ but with a different algebra structure: the Clifford relation

$$
v_{1} v_{2}+v_{2} v_{1}=-2 g\left(v_{1}, v_{2}\right)
$$

holds for two sections $v_{1}$ and $v_{2}$ of $T^{*} Y$. To end this discussion, we give a more practical definition of a $\operatorname{Spin}^{c}$ structure on $(Y, g)$.

Definition 2.1. A $\operatorname{Spin}^{c}$ structure $\mathfrak{s}$ on an oriented, closed 3-manifold $(Y, g)$ is a pair $(W, \rho)$ consisting of a $U(2)$ bundle $W$ and a Clifford multiplication homomorphism 
$\rho: T^{*}(Y) \rightarrow \operatorname{End}(W)$ satisfying the Clifford relation: $\rho\left(v_{1}\right) \rho\left(v_{2}\right)+\rho\left(v_{2}\right) \rho\left(v_{1}\right)=-2 g\left(v_{1}, v_{2}\right)$. and satisfying $\rho\left(e_{1}\right) \rho\left(e_{2}\right) \rho\left(e_{3}\right)=I d_{W}$.

Locally, let $\left\{e^{1}, e^{2}, e^{3}\right\}$ be an oriented orthonormal co-frame for $T^{*} Y$, then the Clifford multiplication can be written as follows

$$
\rho\left(\sum_{i=1}^{3} a_{i} e^{i}\right)=\left(\begin{array}{lr}
a_{1} i & -a_{2}+i a_{3} \\
a_{2}+i a_{3} & -a_{1} i
\end{array}\right) .
$$

The Clifford multiplication homomorphism $\rho$ can be employed to define an $\mathbb{R}$-bilinear form $\sigma: W \otimes W \rightarrow T^{*} Y \otimes i \mathbb{R}$, given by

$$
\begin{aligned}
\sigma(\psi, \psi) & =-\rho^{-1}\left(\psi \otimes \psi^{*}-\frac{|\psi|^{2}}{2} I d\right) \\
& =\frac{i}{2} \operatorname{Im}\left(\left\langle e_{i} \cdot \psi, \psi\right\rangle\right) e^{i} \in \Omega^{1}(Y, i \mathbb{R}) .
\end{aligned}
$$

Here $\psi$ is a section of $W$ and $<,>$ is the Hermitian product on $W$. It is easy to check that $\sigma(\cdot, \cdot)$ satisfies the following properties.

Lemma 2.2. Use . to denote Clifford multiplication, $<,>$ is the Hermitian product on $W$ and $g$ is the Riemannian metric on $Y$, extended linearly to $T^{*} Y \otimes_{\mathbb{R}} \mathbb{C}$, Then

1. $\sigma(\psi, \psi) . \psi=-\frac{1}{2}|\psi|^{2} \psi$, and $|\sigma(\psi, \psi)|^{2}=\frac{1}{4}|\psi|^{4}$.

2. For any imaginary-valued 1-form $\alpha$ on $Y,\langle\alpha . \psi, \psi\rangle=2 g(\alpha, \sigma(\psi, \psi))$ and $\sigma(\alpha . \psi, \phi)+\sigma(\psi, \alpha . \phi)=-(\operatorname{Re}\langle\psi, \phi\rangle) \alpha$.

3. $\sigma(\psi, \phi)=0$ if and only if on $Y \backslash \psi^{-1}(0), \phi=$ ir $\psi$ for a real-valued function $r$ on $Y \backslash \psi^{-1}(0)$.

4. If $\psi$ is a nowhere vanishing section of $W$, then $W \cong \mathbb{C} \psi \oplus \psi^{\perp}$, where $\underline{\mathbb{C}} \psi$ is the trivial complex line bundle generated by $\psi$ and $\psi^{\perp}$ is the orthogonal complement of $\psi$ under the Hermitian product. Moreover, $\sigma(\psi, \cdot)$ defines a bundle isomorphism between $\underline{\mathbb{R}} \psi \oplus \psi^{\perp}$ and $T^{*} Y \otimes i \mathbb{R}$. This last claim implies that a $\operatorname{Spin}^{c}$ structure on $(Y, g)$ can be identified with Turaev's Euler structure 36 on $(Y, g)$.

Proof. Claims (a) and (b) follow from direct calculations. We only prove claim (d), as from (d), we know that the on $Y \backslash \psi^{-1}(0)$, the kernel of the map $\sigma(\psi, \cdot)$ consists of sections of $\underline{i \mathbb{R}} \psi$, and this implies claim (c).

For a nowhere vanishing section $\psi$ of $W$, we write $\psi=|\psi| \tau_{1}$ where $\tau_{1}$ is a unit-length spinor. Choose a local basis $\left\{\tau_{1}, \tau_{2}\right\}$ for the $\operatorname{Spin}^{c}$ bundle, so that Clifford multiplication in the local orthonormal coframe $\left\{e^{1}, e^{2}, e^{3}\right\}$ for $T^{*} Y$ is given by

$$
\rho\left(e_{1}\right)=\left(\begin{array}{rr}
i & 0 \\
0 & -i
\end{array}\right), \rho\left(e_{2}\right)=\left(\begin{array}{rr}
0 & -1 \\
1 & 0
\end{array}\right), \rho\left(e_{3}\right)=\left(\begin{array}{cc}
0 & i \\
i & 0
\end{array}\right) \text {. }
$$


where $\left\{e^{1}, e^{2}, e^{3}\right\}$ can be expressed as

$$
e^{1}=-2 i \sigma\left(\tau_{1}, \tau_{1}\right), e^{2}=2 i \sigma\left(\tau_{1}, i \tau_{1}\right), e^{3}=-2 i \sigma\left(\tau_{1}, \tau_{2}\right) .
$$

Any section of $\underline{\mathbb{R} \psi} \oplus \psi^{\perp}$ can be written as $u \tau_{1}+v \tau_{2}$ for a real-valued function $u$ and a complexvalued function $v$, then

$$
\sigma(\psi, \phi)=\frac{i}{2}|\psi|\left(u e^{1}-\operatorname{Im}(v) e^{2}+\operatorname{Re}(v) e^{3}\right) .
$$

Hence, $\sigma(\psi, \cdot)$ defines a bundle isomorphism between $T^{*} Y \otimes i \mathbb{R}$ and $\underline{\mathbb{R} \psi} \oplus \psi^{\perp}$.

In [36, Turaev defined an Euler structure to be a homology class of a nowhere vanishing vector field on $Y$, where two vector fields are called homologous if they are homotopic away from a point. Identifying $T Y$ with $T^{*} Y$ using the Riemannian metric, for any Euler structure on $(Y, g)$, there is a unit-length section $v$ of $T^{*} Y$ representing that Euler structure, which gives rise to the following decomposition $T^{*} Y=\underline{\mathbb{R} . v} \oplus L$. Then the Hodge star operator can endow $L$ with a complex structure given by $J=*(v \wedge \cdot)$. The corresponding $\operatorname{Spin}^{c}$ structure is given by $W=\underline{\mathbb{C} . v} \oplus L$ and Clifford multiplication by $v$ is given by $\left(\begin{array}{cc}i & 0 \\ 0 & -i\end{array}\right)$.

With the Levi-Civita connection $\nabla$ on the cotangent bundle $T^{*} Y$, a $U(1)$-connection $A$ on the determinant bundle $\operatorname{det}(W)$ determines a connection $\nabla_{A}$ on $W$ such that $\rho$ is parallel, that is, for $v$ and $\psi$ sections of $T^{*} Y$ and $W$ respectively, $\nabla_{A}$ satisfies

$$
\nabla_{A}(v \cdot \psi)=(\nabla v) \cdot \psi+v \cdot \nabla_{A}(\psi)
$$

Then $\nabla_{A}$ is called a Spin ${ }^{c}$ connection on $W$. Applying the Clifford multiplication, we can define a Dirac operator $\not_{A}=\rho \circ \nabla_{A}: \Gamma(W) \rightarrow \Gamma(W)$.

Now we can introduce the Seiberg-Witten equations on $(Y, g, \mathfrak{s})$ for a pair $(A, \psi)$ consisting of a $U(1)$ connection $A$ on the determinant line bundle of $\mathfrak{s}$ and a spinor section $\psi$ of $W$ : 15] [4] [22] [17] [6]:

$$
\left\{\begin{array}{l}
\not \partial_{A} \psi=0 \\
* F_{A}=\sigma(\psi, \psi)+\eta
\end{array}\right.
$$

where $\eta$ is a co-closed, imaginary-valued 1-form on $Y$.

Denote by $\mathcal{A}_{Y}(\mathfrak{s})$ the Seiberg-Witten configuration space on $(Y, \mathfrak{s})$ consisting of pairs $(A, \psi)$ as above Then $\mathcal{A}_{Y}(\mathfrak{s})$ is an affine space modelled on $\Omega^{1}(X, i \mathbb{R}) \times \Gamma(W)$. For analytic reasons, we shall use the $L_{1}^{2}$-integrable configuration space, denoted $\mathcal{A}_{L_{1}^{2}}$, and we require $\eta$ to be $L_{2}^{2}$ integrable, hence $\|\eta\|_{C^{0}}$ is bounded.

The automorphism group of $\operatorname{det}(W)$ is the gauge group $\mathcal{G}_{Y}=\mathcal{G}_{L_{2}^{2}}$ of $L_{2}^{2}$-maps of $Y$ to $U(1)$, locally modelled on the Lie algebra $\Omega_{L_{2}^{2}}^{0}(Y, i \mathbb{R})$. The gauge group $\mathcal{G}_{L_{2}^{2}}$ acts on $\mathcal{A}_{L_{1}^{2}}$ by 
gauge transformations: $(A, \psi) \stackrel{u}{\longmapsto}\left(A-2 u^{-1} d u, u \psi\right)$, which leave the Seiberg-Witten equations invariant. It acts freely on $\{(A, \psi) \mid \psi \neq 0\}$, those elements are said to be irreducible while $(A, \psi)$ with $\psi=0$ is said to be reducible. Denote by $\mathcal{B}=\mathcal{A}_{L_{1}^{2}} / \mathcal{G}_{L_{2}^{2}}$ the quotient of $\mathcal{A}_{L_{1}^{2}}$ by gauge transformations. We use the notation $\mathcal{A}_{L_{1}^{2}}^{*}$ for the open subset of irreducible configurations, and $\mathcal{B}^{*}=\mathcal{A}_{L_{1}^{2}}^{*} / \mathcal{G}_{L_{2}^{2}}$ for the quotient space of $\mathcal{A}_{L_{1}^{2}}^{*}$ by the gauge group $\mathcal{G}_{L_{2}^{2}}$. Then $\mathcal{B}^{*}$ is a Hausdorff space [10], 23], in fact, $\mathcal{B}^{*}$ is a smooth infinite dimensional Hilbert manifold.

At $(A, \psi) \in \mathcal{A}_{L_{1}^{2}}$, the tangent space can be identified with the $L_{1}^{2}$-completion of $\Omega^{1}(Y, i \mathbb{R}) \oplus \Gamma(W)$, we denote this completion by $\Omega_{L_{1}^{2}}^{1}(Y, i \mathbb{R}) \oplus \Gamma_{L_{1}^{2}}(W)$. The infinitesimal action of $\mathcal{G}_{L_{2}^{2}}$ at $(A, \psi)$ defines a map

$$
\begin{array}{ll}
G_{(A, \psi)}: \quad & \Omega_{L_{2}^{2}}^{0}(Y, i \mathbb{R}) \rightarrow \Omega_{L_{1}^{2}}^{1}(Y, i \mathbb{R}) \oplus \Gamma_{L_{1}^{2}}(W) \\
& G_{(A, \psi)}(f)=(-2 d f, f \psi) .
\end{array}
$$

Local slices exist for the action of $\mathcal{G}_{L_{2}^{2}}$ on $\mathcal{A}_{L_{1}^{2}}$. At $[A, \psi] \in \mathcal{B}^{*}$, the tangent space is given by the $L_{1}^{2}$-completion of

$$
\left\{(\alpha, \phi) \in \Omega^{1}(Y, i \mathbb{R}) \oplus \Gamma(W) \mid d^{*} \alpha+i \operatorname{Im}<\psi, \phi>=0\right\}
$$

The $L_{1}^{2}$ metric on $T_{[A, \psi]} \mathcal{B}^{*}$ is defined by the natural $L_{1}^{2}$-norm on one forms and the $L_{1}^{2}$-norm on sections of $W$ using the connection $\nabla_{A}$. This metric is independent of the choice of representative $(A, \psi)$. At a reducible point $[A, 0] \in \mathcal{B}$, there is a neighbourhood of $[A, 0] \in \mathcal{B}$, which is homeomorphic to the quotient of the $L_{1}^{2}$-completion of

$$
\operatorname{Kerd}^{*} \oplus \Gamma(W)
$$

by the action of $U(1)$ on the $\Gamma(W)$ factor. Hence, the reducible point $[A, 0]$ is a singular point in $\mathcal{B}$ with the link of singularity homotopy equivalent to $\mathbb{C} P^{\infty}$.

Definition 2.3. The moduli space $\mathcal{M}_{Y}(\mathfrak{s}, \eta)$ of the Seiberg-Witten monopoles on $(Y, g, \mathfrak{s})$ is the solution space of the Seiberg-Witten equations (9) modulo the gauge transformation group $\mathcal{G}_{L_{2}^{2}}$. The moduli space $\mathcal{M}_{Y}^{*}(\mathfrak{s}, \eta)$ is the irreducible part of $\mathcal{M}_{Y}(\mathfrak{s}, \eta)$.

There is an a priori pointwise estimate to the spinor part of any solution to the SeibergWitten equations (9), which we now state as follows.

Lemma 2.4. Every solution to the Seiberg-Witten equations (9) is gauge equivalent to a $C^{\infty}$ solution. Let $(A, \psi)$ be a smooth solution to the Seiberg-Witten equations (9), then

$$
|\psi|^{2} \leq \max _{y \in Y}\left\{0,-s(y)+2\|\eta\|_{C^{0}}\right\}
$$

where $s(y)$ is the scalar curvature for $(Y, g)$. 
Proof. The first claim follows from standard elliptic regularity theory. The estimate on $|\psi|^{2}$ follows from the Weitzenböck formula (Cf. 15] 23])

$$
\not \partial_{A}^{2}=\nabla_{A}^{*} \nabla_{A}+\frac{s}{4}-\frac{1}{2} \rho\left(* F_{A}\right) .
$$

Let $(A, \psi) \in \mathcal{A}_{L_{1}^{2}}$ be a solution to the Seiberg-Witten equations (9), then there is a natural associated elliptic complex which incorporates the linearization of the gauge action (10) and the linearization of equations (9) as follows. The linearization of the Seiberg-Witten equations (9) at a solution $(A, \psi)$ defines the following map:

$$
\begin{gathered}
L_{(A, \psi)}: \Omega^{1}(Y, i \mathbb{R}) \oplus \Gamma(W) \rightarrow \Omega^{1}(Y, i \mathbb{R}) \oplus \Gamma(W) \\
L_{(A, \psi)}(\alpha, \phi)=\left(* d \alpha-\sigma(\psi, \phi), \not_{A} \phi+\frac{1}{2} \alpha . \psi\right) .
\end{gathered}
$$

The infinitesimal action of $\mathcal{G}_{Y}=\mathcal{G}_{L_{2}^{2}}$ and linearization of the Seiberg-Witten equations at a solution $(A, \psi)$ define the deformation complex

$$
\Omega_{L_{2}^{2}}^{0}(Y, i \mathbb{R}) \stackrel{G_{(A, \psi)}}{\rightarrow} \Omega_{L_{1}^{2}}^{1}(Y, i \mathbb{R}) \oplus \Gamma_{L_{1}^{2}}(W) \stackrel{L_{(A, \psi)}}{\rightarrow} \Omega_{L^{2}}^{1}(Y, i \mathbb{R}) \oplus \Gamma_{L^{2}}(W) .
$$

It is convenient to put the deformation complex (12) in the following form:

$$
\begin{gathered}
\Omega_{L_{1}^{2}}^{1}(Y, i \mathbb{R}) \oplus \Gamma_{L_{1}^{2}}(W) \oplus \Omega_{L_{2}^{2}}^{0}(Y, i \mathbb{R}) \longrightarrow \Omega_{L^{2}}^{1}(Y, i \mathbb{R}) \oplus \Gamma_{L^{2}}(W) \oplus \Omega_{L^{2}}^{0}(Y, i \mathbb{R}) \\
\mathcal{T}_{(A, \psi)}=\left(\begin{array}{cc}
L_{(A, \psi)} & G_{(A, \psi)} \\
G_{(A, \psi)}^{*} & 0
\end{array}\right)
\end{gathered}
$$

where $G_{(A, \psi)}^{*}$ is the dual operator of $G_{(A, \psi)}$ under the $L^{2}$-inner product:

$$
G_{(A, \psi)}^{*}(a, \phi)=-2\left(d^{*} a+i \operatorname{Im}\langle\psi, \phi\rangle\right) .
$$

Note that $\mathcal{T}_{(A, \psi)}$ is a compact perturbation, courtesy of Lemma 2.4 of the sum of the signature operator and the twisted Dirac operator,

$$
\left(\begin{array}{cc}
* d & -2 d \\
-2 d^{*} & 0
\end{array}\right) \oplus \not \partial_{A}
$$

which has index 0 .

Definition 2.5. We say that $[A, \psi]$ in $\mathcal{M}_{Y}(\mathfrak{s}, \eta)$ is non-degenerate if the middle cohomology of (19) is zero:

$$
\operatorname{Ker} L_{(A, \psi)} / \operatorname{Im} G_{(A, \psi)}=0
$$


We summarize the main properties of the monopole moduli space $\mathcal{M}_{Y}(\mathfrak{s}, \eta)$, their proofs can be found in [11] [17] [16] [37.

Proposition 2.6. Let $(Y, g, \mathfrak{s})$ be a closed, Riemannian 3-manifold with a Spin ${ }^{c}$ structure $\mathfrak{s}$, then the Seiberg-Witten moduli space $\mathcal{M}_{Y}(\mathfrak{s}, \eta)$ has the following properties. There exists a Baire set of co-closed 1-forms $\eta \in \Omega_{L_{2}^{2}}^{1}(Y, i \mathbb{R})$ such that all the points in $\mathcal{M}_{Y}(\mathfrak{s}, \eta)$ are non-degenerate. Moreover, if $b_{1}(Y)>0, \mathcal{M}_{Y}(\mathfrak{s}, \eta)$ consists of only finitely many irreducible points; if $Y$ is a rational homology 3-sphere, assume that a generic $\eta$ satisfies $K$ Ker $\not_{\theta}=0$ (where $\theta$ is the unique reducible point in $\mathcal{M}_{Y}(\mathfrak{s}, \eta)$, that is, $\left.* F_{\theta}=\eta\right)$, then $\mathcal{M}_{Y}^{*}(\mathfrak{s}, \eta)=\mathcal{M}_{Y}(\mathfrak{s}, \eta)-\{\theta\}$ consists of only finitely many irreducible points.

Remark 2.7. Proposition 2.0 still holds for a Baire set of co-closed imaginary-valued 1-forms on a fixed non-empty open set $U \subset Y$ (if $b_{1}(Y)>0$ and $c_{1}(\operatorname{det}(\mathfrak{s}))=0, U$ is chosen such that the map: $H_{c p t}^{2}(U, \mathbb{R}) \rightarrow H^{2}(Y, \mathbb{Z})$ is non-trivial). This is established in [1].

\section{$3 \quad$ Seiberg-Witten-Floer homology}

In this Section we will discuss the construction of the Seiberg-Witten-Floer homology groups. The main technical parts have been established in [20]. Here we give another account of analytical parts of the construction of Seiberg-Witten-Floer homology groups and introduce a different perturbation of the Chern-Simons-Dirac functional to achieve transversality for the moduli space of flowlines.

\subsection{The basics of the CSD functional: critical points, relative indices}

Let $Y$ be a closed, oriented 3-manifold with a Riemannian metric $g$ and a $\operatorname{Spin}^{c}$ structure $\mathfrak{s}=(W, \rho)$ on $Y$. Let $\mathcal{A}$ be the $L_{1}^{2}$-configuration space for the Seiberg-Witten equations, the tangent space at any point is the space of $L_{1}^{2}$-sections: $\Omega_{L_{1}^{2}}^{1}(Y, i \mathbb{R}) \oplus L_{1}^{2}(W)$. Let $\mathcal{G}=\mathcal{G}_{Y}$ be the $L_{2}^{2}$ gauge transformation group on $Y$. Its tangent space at the identity is $\Omega_{L_{2}^{2}}^{0}(Y, i \mathbb{R})$ and $\mathcal{G}$ acts on $\mathcal{A}$ with the quotient space denoted by $\mathcal{B}$. For simplicity, we assume that $c_{1}(\operatorname{det}(\mathfrak{s})) \neq 0$ in $H^{2}(Y, \mathbb{R})$ if $b_{1}(Y)>0$.

Fix a $C^{\infty}$ connection $A_{0}$ on $\operatorname{det}(W)$, then there is a Chern-Simons-Dirac function on $\mathcal{A}$ as in [15]:

$$
\mathcal{C}_{\eta}(A, \psi)=-\frac{1}{2} \int_{Y}\left(A-A_{0}\right) \wedge\left(F_{A}+F_{A_{0}}-* 2 \eta\right)+\int_{Y}\left\langle\psi, \not_{A} \psi\right\rangle d v o l_{Y}
$$

Here $\eta$ is a co-closed, imaginary-valued 1-form on $Y$ which is $L_{2}^{2}$-integrable and we can assume that $* \eta$ represents a trivial cohomology class in $H^{1}(Y, i \mathbb{R})$. When $\eta=0$, we denote $\mathcal{C}_{\eta}$ by $\mathcal{C}$. 
For $g \in \mathcal{G}$, we have

$$
\mathcal{C}_{\eta}(g \cdot(A, \psi))-\mathcal{C}_{\eta}(A, \psi)=\left(c_{1}(\operatorname{det}(W)) \wedge[g]\right)([Y]) .
$$

Here $[g]$ is the cohomology class $[g]=\left[-2 \pi i g^{-1} d g\right]$ on $Y$ which defines a homomorphism: $\mathcal{G} \rightarrow H^{1}(Y, \mathbb{Z})$, the kernel of this map is the identity component of $\mathcal{G}$. Therefore, $\mathcal{C}_{\eta}$ descends to a map $\mathcal{B} \rightarrow \mathbb{R} / d(\mathfrak{s}) \mathbb{Z}$ where $d(\mathfrak{s})$ is the divisibility of $c_{1}(\mathfrak{s})=c_{1}(\operatorname{det}(W))$ in $H^{2}(Y, \mathbb{Z}) /$ Torsion:

$$
d(\mathfrak{s})=\text { g.c.d. }\left\{\left\langle c_{1}(\mathfrak{s}), \sigma\right\rangle: \text { for } \sigma \in H_{2}(Y, \mathbb{Z})\right\} .
$$

In the case of $c_{1}(\operatorname{det}(W))=0$, we see from $(15)$ that $\mathcal{C}_{\eta}$ descends to a map $\mathcal{B} \rightarrow \mathbb{R}$. If $c_{1}(\operatorname{det}(W))$ is not a torsion element, we know that $\mathcal{C}_{\eta}$ descends to a map $\mathcal{B} \rightarrow S^{1}$.

With the $L^{2}$-metric on sections of the spinor bundle $W$ given by twice the real part of the $L^{2}$-hermitian product, the gradient of $\mathcal{C}_{\eta}$ at $(A, \psi) \in \mathcal{A}$ is given by

$$
\nabla \mathcal{C}_{\eta}(A, \psi)=\left(* F_{A}-\sigma(\psi, \psi)-\eta, \not_{A} \psi\right)
$$

which is $\mathcal{G}$-equivariant. Hence, $\nabla \mathcal{C}_{\eta}$ defines a section of the $L^{2}$-tangent bundle on $\mathcal{B}^{*}$. Note that the tangent space of $\mathcal{B}^{*}$ at $[A, \psi]$ is the $L_{1}^{2}$-completion of

$$
\operatorname{Ker} G_{[A, \psi]}^{*}=\left\{(a, \phi) \in \Omega^{1}(Y, i \mathbb{R}) \oplus \Gamma(W) \mid d^{*} a+i \operatorname{Im}\langle\psi, \phi\rangle=0\right\} .
$$

The Hessian operator of $\mathcal{C}_{\eta}$ at $[A, \psi] \in \mathcal{B}^{*}$ is given by

$$
\begin{gathered}
Q_{[A, \psi]}: \operatorname{Ker} G_{[A, \psi]}^{*} \longrightarrow \operatorname{Ker}_{[A, \psi]}^{*} \\
Q_{[A, \psi]}:(a, \phi) \mapsto\left(* d a-2 \sigma(\psi, \phi)-2 d f, \not \partial_{A} \phi+\frac{1}{2} a \cdot \psi+f \psi\right)
\end{gathered}
$$

where $f$ is the unique solution in $\Omega_{L_{2}^{2}}^{0}(Y, i \mathbb{R})$ of the equation

$$
\left(d^{*} d+\frac{1}{2}|\psi|^{2}\right) f=i \operatorname{Im}\left\langle\not_{A} \psi, \phi\right\rangle .
$$

Notice that if $[A, \psi]$ is a critical point of $\mathcal{C}_{\eta}$, then $f=0$ by the maximum principle. The following Lemma describes the properties of the Hessian operator $Q_{[A, \psi]}$ of $\mathcal{C}_{\eta}$ at $[A, \psi] \in \mathcal{B}^{*}$.

Lemma 3.1. $Q_{[A, \psi]}$ defines a closed, unbounded, essentially self-adjoint, Fredholm operator on the $L^{2}$-completion of $\operatorname{Ker} G_{[A, \psi]}^{*}$, and its eigenvectors form an $L^{2}$-complete orthonormal basis for $\operatorname{Ker} G_{[A, \psi]}^{*}$. The domain of $Q_{[A, \psi]}$ is the $L_{1}^{2}$-tangent space of $\mathcal{B}^{*}$. The eigenvalues form a discrete subset of the real line which has no accumulation points, and which is unbounded in both directions. Each eigenvalue has finite multiplicity.

Proof. We follow the arguments in [30]. First $Q_{[A, \psi]}$ defines a bounded, essentially self-adjoint, Fredholm operator on the $L_{1}^{2}$-completion of $\operatorname{Ker} G_{[A, \psi]}^{*}$ to the $L^{2}$-completion of $\operatorname{Ker} G_{[A, \psi]}^{*}$. Then 
the forgetful map from $L_{1}^{2}$ to $L^{2}$ is compact and the resolvent of $Q_{[A, \psi]}$ is compact so that $Q_{[A, \psi]}$ has no essential spectrum (accumulation points or isolated eigenvalue with infinite multiplicity). Therefore $Q_{[A, \psi]}$ has discrete spectrum without accumulation points and each eigenvalue has finite multiplicity. The remaining statements are standard in the elliptic theory on compact manifolds.

Let $[A, \psi]$ be a critical point of $\mathcal{C}_{\eta}$ on $\mathcal{B}$, then $[A, \psi]$ is a gauge equivalence class of a solution $(A, \psi) \in \mathcal{A}$ to the Seiberg-Witten equations (9). Hence, the set of critical points of $\mathcal{C}_{\eta}$ on $\mathcal{B}$ is $\mathcal{M}_{Y}(\mathfrak{s}, \eta)$. A critical point $[A, \psi] \in \mathcal{M}_{Y}^{*}(\mathfrak{s}, \eta)$ is called non-degenerate if its Hessian operator is non-degenerate. It is easy to see that a non-degenerate critical point is isolated in $\mathcal{M}_{Y}(\mathfrak{s}, \eta)$. A reducible critical point is of the form $[A, 0]$ with $* F_{A}=\eta$. For generic $\eta, * F_{A}=\eta$ admits solutions only when $Y$ is a rational homology 3 -sphere. We call a reducible critical point nondegenerate if it is isolated in $\mathcal{M}_{Y}(\mathfrak{s}, \eta)$. From Proposition 2.6, we obtain the following property of this critical point set.

Lemma 3.2. For generic $\eta$, the critical points of $\mathcal{C}_{\eta}$ on $\mathcal{B}$ are all non-degenerate.

Proof. For an irreducible critical point $c \in \mathcal{M}_{Y}(\mathfrak{s}, \eta)$, choose a $C^{\infty}$ solution $(A, \psi)$ to (9) which represents $c$. The Hessian operator $Q_{c}$ has a $\mathcal{G}$-equivariant extension given by (13), namely

$$
\mathcal{T}_{(A, \psi)}=\left(\begin{array}{cc}
L_{(A, \psi)} & G_{(A, \psi)} \\
G_{(A, \psi)}^{*} & 0
\end{array}\right) .
$$

By direct calculation, we obtain

$$
\operatorname{Ker} Q_{c} \cong \operatorname{Ker} \mathcal{T}_{(A, \psi)} \cong \operatorname{Ker} L_{(A, \psi)} / \operatorname{Im} G_{(A, \psi)} .
$$

Then Proposition 2.6 implies that $K e r Q_{c}=0$ for generic $\eta$.

If $\theta=[A, 0]$ is a critical point of $\mathcal{C}_{\eta}$ on $\mathcal{B}$, which only happens when $Y$ is a rational homology 3sphere $\left(* F_{A}=\eta\right)$, then one can check that under the condition $\operatorname{Ker} \not_{A}=0, \operatorname{Ker} L_{(A, 0)} / \operatorname{Im} G_{(A, 0)}$ is zero for the deformation complex (13) at $(A, 0)$, hence $\theta$ is also non-degenerate.

Henceforth $\mathcal{M}_{Y}(\mathfrak{s}, \eta)$ denotes the set of critical points of $\mathcal{C}_{\eta}$ which are assumed all nondegenerate.

There are two fundamental lemmata concerning the gradient of the Chern-Simons-Dirac functional and the estimate of local distance near a critical point. The first of these shows that $\mathcal{C}_{\eta}$ satisfies the Palais-Smale condition which was proved for the unperturbed case $(\eta=0)$ in 25] [13. The proof here follows the arguments from [25]. 
Lemma 3.3. For any $\epsilon>0$, there is $\lambda>0$ such that if $[A, \psi] \in \mathcal{B}$ has $L_{1}^{2}$-distance at least $\epsilon$ from all the critical points in $\mathcal{M}_{Y}(\mathfrak{s}, \eta)$, then

$$
\left\|\nabla \mathcal{C}_{\eta, \nu}([A, \psi])\right\|_{L^{2}}>\lambda
$$

Proof. Suppose that, on the contrary, there is a sequence $c_{i}$ in $\mathcal{B}^{*}$ whose $L_{1}^{2}$-distance from $c_{i}$ to the set of critical points is at least $\epsilon$, for which $\left\|\nabla \mathcal{C}_{\eta}\left(c_{i}\right)\right\|_{L^{2}} \rightarrow 0$ as $i \rightarrow \infty$. Choose a sequence of $\left(A_{i}, \psi_{i}\right)$ representing $c_{i}$, then there is a constant $C$ such that

$$
\int_{Y}\left(\left|* F_{A_{i}}-\sigma\left(\psi_{i}, \psi_{i}\right)-\eta\right|^{2}+2\left|\not_{A_{i}} \psi_{A_{i}}\right|^{2}\right) d v o l \leq C .
$$

Using the Weitzenböck formula and Lemma 2.2, we have

$$
\left\|F_{A_{i}}\right\|_{L^{2}}^{2}+\left\|\sigma\left(\psi_{i}, \psi_{i}\right)+\eta\right\|_{L^{2}}^{2}-2<* F_{A_{i}}, \eta>+2\left\|\nabla_{A_{i}} \psi\right\|_{L^{2}}^{2}+\frac{s}{2}\left\|\psi_{i}\right\|_{L^{2}}^{2} \leq C,
$$

where $s$ is the scalar curvature on $(Y, g)$. Applying the inequality $2|a b| \leq \frac{1}{2}|a|^{2}+2|b|^{2}$ and the Cauchy-Schwartz inequality, we can rewrite the above expression as

$$
\begin{aligned}
& \frac{1}{2}\left\|F_{A_{i}}\right\|_{L^{2}}^{2}+\frac{1}{8}\left\|\psi_{i}\right\|_{L^{4}}^{4}+2\left\|\nabla_{A_{i}} \psi\right\|_{L^{2}}^{2} \\
\leq & C+3\|\eta\|_{L^{2}}^{2}+\max _{y \in Y}\left\{-\frac{s(y)}{2}\right\} \sqrt{\operatorname{Vol}(Y)}\left\|\psi_{i}\right\|_{L^{4}}^{2} \\
\leq & C+3\|\eta\|_{L^{2}}^{2}+\frac{1}{16}\left\|\psi_{i}\right\|_{L^{4}}^{4}+\max _{y \in Y}\left\{-\frac{|s(y)|^{2}}{2}\right\} \operatorname{Vol}(Y) .
\end{aligned}
$$

From this inequality, we see that $\left\|F_{A_{i}}\right\|_{L^{2}},\left\|\psi_{i}\right\|_{L^{4}}$ and $\left\|\nabla_{A_{i}} \psi\right\|_{L^{2}}$ are all bounded independent of $i$. By standard elliptic regularity theory we know that there exists a subsequence of $\left\{A_{i}, \psi_{i}\right\}$, such that $\left(A_{i}, \psi_{i}\right)$ converges in $L_{1}^{2}$-topology to a limit $\left(A_{\infty}, \psi_{\infty}\right)$ which is a solution to the Seiberg-Witten equations (9) and hence represents a critical point in $\mathcal{M}_{Y}(\mathfrak{s}, \eta)$. This contradicts the fact that $\left\{\left[A_{i}, \psi_{i}\right]\right\}$ has distance at least $\epsilon$ from the critical points.

Remark 3.4. Note that $\lambda$ is independent of $\eta$ when $\eta$ is sufficiently small, hence from now on, we choose a sufficiently small $\epsilon_{0}<<1$ and $\eta$ to have $L^{2}$-norm less than $\epsilon_{0} \lambda$.

The second lemma is the following $L_{1}^{2}$-distance estimate near the non-degenerate critical points of $\mathcal{C}_{\eta}$, which was also established in 25].

Lemma 3.5. Suppose $\alpha$ is a non-degenerate critical point of $\mathcal{C}_{\eta}$. There exists a positive constant $C_{\alpha}$ and an $\epsilon>0$, depending on $\alpha$, such that if the $L_{1}^{2}$-distance from $[A, \psi]$ to $\alpha$ is less than $\epsilon$, then the $L_{1}^{2}$-distance from $[A, \psi]$ to $\alpha$ (denoted by $\operatorname{dist}_{L_{1}^{2}}([A, \psi], \alpha)$ ) is bounded by

$$
C_{\alpha}\left\|\nabla \mathcal{C}_{\eta, \nu}(A, \psi)\right\|_{L^{2}}
$$


Proof. When $\operatorname{dist}_{L_{1}^{2}}([A, \psi], \alpha)$ is sufficiently small, there are representatives $(A, \psi)$ and $\left(A_{\alpha}, \psi_{\alpha}\right)$ for $[A, \psi]$ and $\alpha$ respectively such that $(A, \psi)=\left(A_{\alpha}, \psi_{\alpha}\right)+(b, \phi)$ with $(b, \phi) \in T_{\alpha} \mathcal{B}^{*}$ with $L_{1}^{2}$-norm less than some $\epsilon$ (to be determined). Then

$$
\nabla \mathcal{C}_{\eta}(A, \psi)=Q_{\alpha}(b, \phi)+N(b, \phi),
$$

where $N(b, \phi)$ is a quadratic term in $(b, \phi)$, satisfying $\|N(b, \phi)\|_{L^{2}} \leq C \epsilon\|(b, \phi)\|_{L_{1}^{2}}$ where $C$ is the universal constant for the Sobolev multiplication theorem. As $Q_{\alpha}$ is non-degenerate, let $\lambda_{1}$ be the first absolute eigenvalue of $Q_{\alpha}$ (Cf. Lemma 3.1). We then obtain

$$
\begin{aligned}
\lambda_{1}\|(b, \phi)\|_{L_{1}^{2}} & \leq\left\|Q_{\alpha}(b, \phi)\right\|_{L^{2}} \\
& \leq\left\|\nabla \mathcal{C}_{\eta}(A, \psi)\right\|_{L^{2}}+\|N(b, \phi)\|_{L^{2}} \\
& \leq\left\|\nabla \mathcal{C}_{\eta}(A, \psi)\right\|_{L^{2}}+C \epsilon\|(b, \phi)\|_{L_{1}^{2}} .
\end{aligned}
$$

Choose $\epsilon<\frac{\lambda_{1}}{2 C}$, then

$$
\|(b, \phi)\|_{L_{1}^{2}} \leq \frac{1}{\lambda_{1}-C \epsilon}\left\|\nabla \mathcal{C}_{\eta}(A, \psi)\right\|_{L^{2}} \leq \frac{2}{\lambda_{1}}\left\|\nabla \mathcal{C}_{\eta}(A, \psi)\right\|_{L^{2}} .
$$

Set $C_{\alpha}=\frac{2}{\lambda_{1}}$, we get $\operatorname{dist}_{L_{1}^{2}}([A, \psi], \alpha) \leq C_{\alpha}\left\|\nabla \mathcal{C}_{\eta}(A, \psi)\right\|_{L^{2}}$.

For any two irreducible critical points $\left[A_{0}, \psi_{0}\right]$ and $\left[A_{1}, \psi_{1}\right]$, choose a path $\left[A_{t}, \psi_{t}\right]$ on $\mathcal{B}^{*}$ connecting $\left[A_{0}, \psi_{0}\right]$ to $\left[A_{1}, \psi_{1}\right]$. Then by Lemma 3.1, $Q_{\left[A_{t}, \psi_{t}\right]}$ defines a path of self-adjoint Fredholm operators, each of which has eigenvalues forming a discrete subset of the real line with no accumulation points. The spectral flow of $Q_{\left[A_{t}, \psi_{t}\right]}$ along this path is defined to be the intersection number of the spectrum graph

$$
\bigcup_{t \in[0,1]} \operatorname{Spec} Q_{\left[A_{t}, \psi_{t}\right]}
$$

with the line $\epsilon=0$ (Cf. 酒). The spectral flow of the Hessian operator defines a locally constant function on the space of continuous paths in $\mathcal{B}^{*}$ between $\left[A_{0}, \psi_{0}\right]$ and $\left[A_{1}, \psi_{1}\right]$ depending only on the homotopy class of the path between $\left[A_{0}, \psi_{0}\right]$ and $\left[A_{1}, \psi_{1}\right]$. By the Atiyah-Patodi-Singer index theorem, its mod $(d(\mathfrak{s}))$ reduction doesn't depend on the homotopy class of the path, where $d(\mathfrak{s})$ is the divisibility of the first Chern class $c_{1}(\operatorname{det}(W))$ in $H^{2}(Y, \mathbb{Z}) /$ Torsion.

We know that $Q_{[A, \psi]}$ has a $\mathcal{G}$-equivariant extension $\mathcal{T}_{(A, \psi)}$ referred to as the extended Hessian operator at a smooth solution $(A, \psi)$ to the Seiberg-Witten equations (9) on $\mathcal{A}$ representing $[A, \psi]$. Let $\left(A_{0}, \psi_{0}\right)$ and $\left(A_{1}, \psi_{1}\right)$ represent two irreducible critical points $\left[A_{0}, \psi_{0}\right]$ and $\left[A_{1}, \psi_{1}\right]$ in $\mathcal{M}_{Y}(\mathfrak{s}, \eta)$ respectively. By its construction, the kernel of $\mathcal{T}_{\left(A_{i}, \psi_{i}\right)}$ is trivial, and so the spectral flow of $T_{(A, \psi)}$ from $\left(A_{0}, \psi_{0}\right)$ to $\left(A_{1}, \psi_{1}\right)$ is a well defined $\mathbb{Z}$-valued function and

$$
S F_{\left[A_{0}, \psi_{0}\right]}^{\left[A_{1}, \psi_{1}\right]} Q=S F_{\left(A_{0}, \psi_{0}\right)}^{\left(A_{1}, \psi_{1}\right)} \mathcal{T}(\bmod d(\mathfrak{s}))
$$


For a homology 3 -sphere $Y$, we need to choose the perturbation $\eta$ so that the unique reducible critical point $\theta$ is isolated, that is, $(g, \eta)$ is away from the codimension one set (Cf. [20]) where the corresponding Dirac operator has non-trivial kernel. Then we can replace $\left[A_{0}, \psi_{0}\right]$ by $\theta$ in (16). Now $\mathcal{T}$ has one dimensional kernel at $\theta$, therefore the spectral flow of $\mathcal{T}$ from $\theta$ to an irreducible critical point is defined to be the intersection number of the eigenvalues with the line $\lambda=-\epsilon$ where $\epsilon$ is any sufficiently small positive number (see [1] ).

Therefore, using the spectral flow of the Hessian operator, we can define a relative index map on the critical point set $\mathcal{M}_{Y}(\mathfrak{s}, \eta)$.

Definition 3.6. Using the spectral flow of the Hessian operator of the Chern-Simons-Dirac functional, there is a relative index map on the critical point set $\mathcal{M}_{Y}(\mathfrak{s}, \eta)$ :

$$
\mathfrak{i}: \quad \mathcal{M}_{Y}(\mathfrak{s}, \eta) \times \mathcal{M}_{Y}(\mathfrak{s}, \eta) \longrightarrow \mathbb{Z}_{d(\mathfrak{s})}
$$

which is the spectral flow $(\bmod d(\mathfrak{s}))$ of the extended Hessian operator along any path connecting two smooth solutions to (9) representing two critical points in $\mathcal{B}$. When $c_{1}(\mathfrak{s})$ is a torsion class (as occurs for example when $Y$ is a homology 3-sphere) then $\mathfrak{i}$ is an integer-valued function.

\subsection{Estimates on gradient flowlines}

The downward gradient flow equation for $\mathcal{C}_{\eta}$ on $\mathcal{A}$ is by definition given by the paths of pairs $(A(t), \psi(t))$ that satisfy the equations

$$
\left\{\begin{array}{l}
\frac{d A(t)}{d t}=-* F_{A(t)}+\sigma(\psi, \psi)+\eta, \\
\frac{d \psi(t)}{d t}=-\partial_{A(t)} \psi(t) .
\end{array}\right.
$$

Using the projection $\pi: Y \times \mathbb{R} \rightarrow Y$, we identify $\Omega^{2,+}(Y \times \mathbb{R}, i \mathbb{R}) \cong \pi^{*}\left(\Omega^{1}(Y, i \mathbb{R})\right)$ by sending $\rho(t)$ to $\frac{1}{2}(* \rho(t)+\rho(t) \wedge d t)$, and identify $\Omega^{1}(Y \times \mathbb{R}, i \mathbb{R}) \cong \pi^{*}\left(\Omega^{0}(Y, i \mathbb{R}) \oplus \Omega^{1}(Y, i \mathbb{R})\right)$ by sending $(f(t), \rho(t))$ to $\rho(t)+f(t) d t$. Using Clifford multiplication by $d t$, we can identify the positive and negative spinor bundles $W^{ \pm}$both as $\pi^{*}(W)$ via $\rho^{ \pm}$, such that for any 1 -form $a \in \Omega^{1}(Y, i \mathbb{R})$ and $\psi \in W$, we have $\rho^{+}(a . \psi)=a . d t . \rho^{+}(\psi)=a . \rho^{-}(\psi)$.

Let $\psi(t)$ be a section of $\pi^{*}(W)$ and $A(t)$ be a family of connections on $\operatorname{det}(W)$. Then we can view $A(t)$ as a connection on $\operatorname{det}\left(\pi^{*} W\right)$, and the twisted Dirac operator $\not D_{A}$ for $\left(Y \times \mathbb{R}, \pi^{*}(W)\right)$ can be expressed as $\partial_{t}+\not \partial_{A(t)}$ in the sense of

$$
\not D_{A}\left(\rho^{+} \psi\right)=\rho^{-}\left(\left(\partial_{t}+\not \partial_{A(t)}\right) \psi(t)\right)
$$

Then, as discovered by Kronheimer-Mrowka [15], the downward gradient flow equations (18) are the following Seiberg-Witten equations on $\left(Y \times \mathbb{R}, \pi^{*}(W)\right)$ in temporal gauge (the $d t$ 
component of $U(1)$-connection on the determinant bundle vanishes identically).

$$
\left\{\begin{array}{l}
F_{A}^{+}=q(\psi, \psi)+\eta^{+} \\
\not D_{A} \psi=0
\end{array}\right.
$$

where $F_{A}^{+}$is the self-dual part of the curvature $F_{A}, \eta^{+}=\frac{1}{2}\left(*_{3} \eta+\eta \wedge d t\right)$, and $q(\psi, \psi)$ is the self-dual 2-form given by

$$
q(\psi, \psi)=(d t \wedge \sigma(\psi, \psi))^{+}=\frac{1}{2}\left(d t \wedge \sigma(\psi, \psi)+*_{3} \sigma(\psi, \psi)\right) .
$$

Two solutions to the equations (19) are gauge equivalent if the paths in $\mathcal{A}$ they determine in temporal gauge are gauge equivalent under the gauge group $\mathcal{G}_{Y}=\operatorname{Map}(Y, U(1))$.

For any solution $S(t)=(A(t), \psi(t))$ of the downward gradient flow equations (18), we have, for any $t_{1}<t_{2}$,

$$
\begin{aligned}
& \mathcal{C}_{\eta}\left(S\left(t_{1}\right)\right)-\mathcal{C}_{\eta}\left(S\left(t_{2}\right)\right) \\
= & -\int_{t_{1}}^{t_{2}}<\nabla \mathcal{C}_{\eta}\left((S(t)), \frac{\partial S(t)}{\partial t}>_{L^{2}} d t\right. \\
= & \int_{t_{1}}^{t_{2}} d t \int_{Y}\left(\left|\frac{\partial A(t)}{\partial t}\right|^{2}+2\left|\frac{\partial \psi(t)}{\partial t}\right|^{2}\right) d v o l_{Y} \\
= & \int_{t_{1}}^{t_{2}} \int_{Y}\left(\left|* F_{A}-\sigma(\psi, \psi)-\eta\right|^{2}+2\left|\not \partial_{A} \psi\right|^{2}\right) d v o l_{Y} \\
= & \int_{t_{1}}^{t_{2}} d t \int_{Y}\left(\left|F_{A}\right|^{2}+|\sigma(\psi, \psi)+\eta|^{2}-2<* F_{A}, \eta>+2\left|\nabla_{A} \psi\right|^{2}+\frac{s}{2}|\psi|^{2}\right) d v o l_{Y} \\
= & \int_{t_{1}}^{t_{2}} d t \int_{Y}\left(\left|F_{A}\right|^{2}+|\sigma(\psi, \psi)+\eta|^{2}+2\left(F_{A} \wedge \eta\right)+2\left|\nabla_{A} \psi\right|^{2}+\frac{s}{2}|\psi|^{2}\right) d v o l_{Y}
\end{aligned}
$$

where $F_{A}$ is the curvature of $A(t)$ on a $t$-slice of $\left[t_{1}, t_{2}\right] \times Y, *$ is the Hodge star operator on $(Y, g), \nabla_{A}$ is the covariant derivative on $W$ over the $t$-slice of $\left[t_{1}, t_{2}\right] \times Y$ and all the norms are on the $t$-slice.

Note that $A(t)$ can be viewed as a connection on the determinant line bundle of the pull-back $\operatorname{Spin}^{c}$ structure on $\left[t_{1}, t_{2}\right] \times Y$. We will write $\mathbb{A}=A(t)$ for this connection on the determinant line bundle over $\left[t_{1}, t_{2}\right] \times Y$ to distinguish it from $A(t)$ viewed as a connection on the determinant line bundle over the $t$-slice of $\left[t_{1}, t_{2}\right] \times Y$. Similarly, denote the corresponding section of the pull-back $\operatorname{Spin}^{c}$ bundle over $\left[t_{1}, t_{2}\right] \times Y$ by $\Psi=\psi(t)$. If $(A(t), \psi(t))$ is a solution to the gradient flow equation (18), then $(\mathbb{A}, \Psi)$ is a solution to the 4-dimensional Seiberg-Witten equations (19) on $\left[t_{1}, t_{2}\right] \times Y$. With this notation understood, the last integration in (20) has a 4-dimensional analogue, called the energy of the 4 -dimensional monopole. We denote it by $E_{\left[t_{1}, t_{2}\right] \times Y}(\mathbb{A}, \Psi)$ and define it as follows.

$$
\begin{aligned}
& E_{\left[t_{1}, t_{2}\right] \times Y}(\mathbb{A}, \Psi) \\
= & \int_{\left[t_{1}, t_{2}\right] \times Y}\left(\left|F_{\mathbb{A}}\right|^{2}+2\left|q(\Psi, \Psi)+\eta^{+}\right|^{2}+2\left|\nabla_{\mathbb{A}} \Psi\right|^{2}+\frac{s}{2}|\Psi|^{2}\right) d v o l+2\left(F_{\mathbb{A}} \wedge d t \wedge \eta\right)
\end{aligned}
$$


Since

$$
\left|F_{\mathbb{A}}\right|^{2}=\left|F_{A}\right|^{2}+\left|\frac{\partial A(t)}{\partial t}\right|^{2},\left|\nabla_{\mathbb{A}} \Psi\right|^{2}=\left|\nabla_{A} \psi\right|^{2}+\left|\frac{\partial \psi(t)}{\partial t}\right|^{2}
$$

and

$$
2\left|q(\Psi, \Psi)+\eta^{+}\right|^{2}=|\sigma(\psi, \psi)+\eta|^{2}
$$

we know that

$$
E_{\left[t_{1}, t_{2}\right] \times Y}(\mathbb{A}, \Psi)=2\left(\mathcal{C}_{\eta}\left(S\left(t_{1}\right)\right)-\mathcal{C}_{\eta}\left(S\left(t_{2}\right)\right)\right)
$$

Hence we will say that any gradient flow line $S(t)=(A(t), \psi(t))\left(t \in\left[t_{1}, t_{2}\right]\right)$ on $\mathcal{A}$ has a finite variation of $\mathcal{C}_{\eta}$ if $S(t)$ satisfies

$$
\mathcal{C}_{\eta}\left(S\left(t_{1}\right)\right)-\mathcal{C}_{\eta}\left(S\left(t_{2}\right)\right)=\int_{t_{1}}^{t_{2}}\left(\left\|\frac{\partial A(t)}{\partial t}\right\|_{L^{2}(Y)}^{2}+2\left\|\frac{\partial \psi(t)}{\partial t}\right\|_{L^{2}(Y)}^{2}\right) d t<\infty .
$$

That is, the corresponding 4-dimensional monopole $(\mathbb{A}, \Psi)$ has finite energy over $\left[t_{1}, t_{2}\right] \times Y$, or equivalently

$$
\int_{t_{1}}^{t_{2}}\left\|\nabla \mathcal{C}_{\eta}(S(t))\right\|_{L^{2}(Y)}^{2} d t=\int_{t_{1}}^{t_{2}}\left(\left\|\frac{\partial A(t)}{\partial t}\right\|_{L^{2}(Y)}^{2}+\left\|\frac{\partial \psi(t)}{\partial t}\right\|_{L^{2}(Y)}^{2}\right) d t<\infty .
$$

Lemma 3.7. Suppose $(\mathbb{A}, \Psi)$ to be a solution to the Seiberg-Witten equations (19) on $[t-2, t+2] \times Y$ with finite energy

$$
E_{[t-2, t+2] \times Y}(\mathbb{A}, \Psi)<E_{0} .
$$

For any positive integer $k$, there exists a constant $C$ depending on the metric on $Y$ and the perturbation term $\eta$ such that all the $C^{k}$-norms of $F_{\mathbb{A}}$ and $\Psi$ on $\left[t-\frac{3}{2}, t+\frac{3}{2}\right] \times Y$ are bounded by $C\left(\sqrt{E_{0}}+1\right)$.

Proof. Let $N_{s}=[t-s, t+s] \times Y$. From $E_{N_{2}}(\mathbb{A}, \Psi)<E_{0}$, we immediately have

$$
\begin{aligned}
& \left\|F_{\mathbb{A}}\right\|_{L^{2}\left(N_{2}\right)}^{2} \leq \frac{E_{0}}{4}+C_{0}, \\
& \|\Psi\|_{L^{4}\left(N_{2}\right)}^{4} \leq 4 E_{0}+C_{0}, \\
& \left\|\nabla_{\mathbb{A}} \Psi\right\|_{L^{2}\left(N_{2}\right)}^{2} \leq \frac{E_{0}}{4}+C_{0},
\end{aligned}
$$

for some constant $C_{0}$ depending on the metric on $Y$ and $\eta$. The standard bootstrapping argument in elliptic regularity theory (Cf. [23]) implies that the $C^{k}$-norms of $F_{\mathbb{A}}$ and $\Psi$ on $\left[t-\frac{3}{2}, t+\frac{3}{2}\right] \times Y$ are bounded by $C\left(\sqrt{E_{0}}+1\right)$ for some constant $C_{0}$ depending on the metric on $Y$ and $\eta$. 
Lemma 3.8. Given a sufficiently small $\epsilon>0$, there exists a constant $E_{0}>0$ such that, for a solution $(A(t), \psi(t))$ to the Seiberg-Witten equations (19) on $[T-2, T+2] \times Y$ in temporal gauge, with finite energy $E_{[T-2, T+2] \times Y}(A(t), \psi(t))<E_{0}$, there exists a critical point $\alpha$ in $\mathcal{M}_{Y}(\mathfrak{s}, \eta)$ with $L_{1}^{2}$-distance from $[A(t), \psi(t)] \in \mathcal{B}$ to $\alpha$ less than $\epsilon$ for any $t \in[T-1, T+1]$.

Proof. The critical points of $\mathcal{M}_{Y}(\mathfrak{s}, \eta)$ are non-degenerate and hence isolated. Given an $\epsilon$ sufficiently less than $\min \left\{\operatorname{dist}_{L_{1}^{2}}(\alpha, \beta): \alpha, \beta \in \mathcal{M}_{Y}(\mathfrak{s}, \eta)\right\}$, let $\lambda$ be the constant in Lemma 3.3 such that if $[A, \psi]$ has $L_{1}^{2}$-distance at least $\epsilon$ to any critical point in $\mathcal{M}_{Y}(\mathfrak{s}, \eta)$, then $\left\|\nabla \mathcal{C}_{\eta}([A, \psi])\right\|_{L^{2}(Y)}>\lambda$. Now we only need to show that for any $t \in[T-1, T+1]$, $\left\|\nabla \mathcal{C}_{\eta}([A(t), \psi(t)])\right\|_{L^{2}(Y)}<\lambda$.

Let $N_{s}=[T-s, T+s] \times Y$ and $(\dot{A}, \dot{\psi})=\left(\frac{\partial A(t)}{\partial t}, \frac{\partial \psi(t)}{\partial t}\right)$. Differentiating the Seiberg-Witten equations (19) and noting that $\left(\frac{\partial A(t)}{\partial t}, \frac{\partial \psi(t)}{\partial t}\right)=-\nabla \mathcal{C}_{\eta}(A, \psi)$ is $L^{2}$-orthogonal to the gauge orbit at each point, we obtain the following elliptic system of equations on $[T-2, T+2] \times Y$,

$$
\left\{\begin{array}{l}
P_{+}(d \dot{A})-2 q(\dot{\psi}, \psi)=0 \\
d^{*} \dot{A}+i I m<\dot{\psi}, \psi>=0 \\
\not D_{A} \dot{\psi}+\frac{1}{2} \dot{A} \cdot \psi=0 .
\end{array}\right.
$$

We deduce from this system of equations and the estimates on $\psi$ in Lemma 3.7 that

$$
\begin{aligned}
\|(\dot{A}, \dot{\psi})\|_{L_{1}^{2}\left(N_{\frac{3}{2}}\right)} & \leq C_{0}\left(\|(\dot{A}, \dot{\psi})\|_{L_{1}^{2}\left(N_{\frac{3}{2}}\right)}+\left\|\left(P_{+}(d \dot{A}), d^{*} \dot{A}, \not D_{A} \dot{\psi}\right)\right\|_{L_{1}^{2}\left(N_{2}\right)}\right) \\
& \leq C_{0}\left(\|(\dot{A}, \dot{\psi})\|_{L_{1}^{2}\left(N_{\frac{3}{2}}\right.}+\left\|\left(q(\dot{\psi}, \psi), i \operatorname{Im}<\dot{\psi}, \psi>, \frac{1}{2} \dot{A} \cdot \psi\right)\right\|_{L_{1}^{2}\left(N_{2}\right)}\right) \\
& \leq C_{1}\left(\sqrt{E_{0}}+\|\psi\|_{C^{0}}\|(\dot{A}, \dot{\psi})\|_{L_{1}^{2}\left(N_{2}\right)}\right) \\
& \leq C_{1}\left(\sqrt{E_{0}}+C_{2}\left(\sqrt{E_{0}}+1\right) \sqrt{E_{0}}\right) \\
& \leq C_{3} \sqrt{E_{0}}
\end{aligned}
$$

Here the $C_{i}$ 's are constants depending on the metric on $Y$ and $\eta$, and $\sqrt{E_{0}}$ bounds the energy of $(A(t), \psi(t))$. Therefore, from the standard elliptic regularity theory of Degiorgi-Nash-Moser (35] Page 169-179), we get that for any $t \in[T-1, T+1]$,

$$
\left\|\nabla \mathcal{C}_{\eta}([A(t), \psi(t)])\right\|_{L^{2}(Y)}=\|(\dot{A}, \dot{\psi})\|_{L^{2}(\{t\} \times Y)} \leq C_{4}\|(\dot{A}, \dot{\psi})\|_{L_{1}^{2}\left(N_{\frac{3}{2}}\right.} \leq C_{5} \sqrt{E_{0}} .
$$

Now we can choose our solution $(A(t), \psi(t))$ so that its energy is bounded by $E_{0}$ with $C_{5} \sqrt{E_{0}} \leq \epsilon$, then the claim in the lemma follows.

We remark that standard elliptic regularity theory implies that $[A(t), \psi(t)]$ has, for all $k$, $C^{k}$-distance $\epsilon$ or less to a critical point in $\mathcal{M}_{Y}(\mathfrak{s}, \eta)$ for any $t \in[T-1, T+1]$. The claims in 
Lemma 3.8 still hold for a solution to the Seiberg-Witten equations on any compact set times $Y$ with sufficiently small energy.

Now we can establish the crucial lemma concerning the exponential decay estimate on a flowline with small energy.

Proposition 3.9. There is a constant $\delta>0$, such that a choice of sufficiently small $\epsilon$ determines $E_{0}>0$ with the following property: suppose that $(A(t), \psi(t))$ is a solution to the Seiberg-Witten equations on $\left[R_{1}-2, R_{2}+2\right] \times Y\left(R_{1}<R_{2}\right)$ in temporal gauge with energy

$$
E_{\left[R_{1}-2, R_{2}+2\right] \times Y}(A(t), \psi(t))<E_{0}
$$

then there exists a critical point $\alpha \in \mathcal{M}_{Y}(\mathfrak{s}, \eta)$ such that the $L_{1}^{2}$-distance from $[A(t), \psi(t)]$ (for any $\left.t \in\left[R_{1}, R_{2}\right]\right)$ to $\alpha$ is at most $4 \epsilon\left(e^{-\delta\left(t-R_{1}\right)}+e^{-\delta\left(R_{2}-t\right)}\right)$. Furthermore, there exists a sequence of constants $\left\{\lambda_{k}\right\}_{k=0,1,2, \ldots}$ such that there is a point $\left(A_{\alpha}, \psi_{\alpha}\right)$ representing $\alpha$ for which

$$
\left|\nabla^{k}\left(A(t)-A_{\alpha}\right)\right|+\left|\left(\nabla_{A_{\alpha}}\right)^{k}\left(\psi(t)-\psi_{\alpha}\right)\right| \leq \lambda_{k}\left(e^{-\delta\left(t-R_{1}\right)}+e^{-\delta\left(R_{2}-t\right)}\right),
$$

for any point $(t, y) \in\left[R_{1}, R_{2}\right] \times Y$.

Proof. We can choose $\epsilon>0$ and $E_{0}>0$ as in Lemma 3.8, so that $\operatorname{dist}_{L_{1}^{2}}([A(t), \psi(t)], \alpha)<\epsilon$ for some critical point $\alpha \in \mathcal{M}_{Y}(\mathfrak{s}, \eta)$ and any $t \in\left[R_{1}-1, R_{2}+1\right]$. Then, on $\left[R_{1}-1, R_{2}+1\right] \times Y$, we can write

$$
(A(t), \psi(t))=\left(A_{\alpha}, \psi_{\alpha}\right)+(b, \phi)
$$

for some representative $\left(A_{\alpha}, \psi_{\alpha}\right)$ of $\alpha$, and $(b, \phi)$ has $C^{1}$-norm less than $\epsilon$ and satisfies $d^{*} b+i \operatorname{Im}<\psi_{\alpha}, \phi>=0$. As $(A(t), \psi(t))$ is a solution to the Seiberg-Witten equations on $\left[R_{1}-1, R_{2}+1\right] \times Y$, the pair $(b, \phi)$ must satisfy

$$
\partial_{t}(b, \phi)+Q_{\alpha}(b, \phi)+N(b, \phi)=0
$$

where $Q_{\alpha}(b, \phi)=\left(* d b-2 \sigma\left(\psi_{\alpha}, \phi\right), \not_{A_{\alpha}} \phi+\frac{1}{2} b . \psi_{\alpha}\right)$ and $N(b, \phi)=\left(-\sigma(\phi, \phi), \frac{1}{2} b . \phi\right)$.

Note that $Q_{\alpha}$ is the Hessian operator of $\mathcal{C}_{\eta}$ at $\alpha$, whose $L^{2}$ spectrum is discrete, real and without accumulation points (Cf. Lemma 3.1). The non-degeneracy of $\alpha$ implies that 0 is not in the spectrum of $Q_{\alpha}$. Let $\mu_{\alpha}$ be the distance between 0 and the spectrum of $Q_{\alpha}$. On the $t$-slice of $\left[R_{1}-1, R_{2}+1\right] \times Y$,

$$
\|N(b, \phi)\|_{L_{1}^{2}(\{T\} \times Y)}<\epsilon\|(b, \phi)\|_{L_{1}^{2}(\{T\} \times Y)} .
$$

Let $\lambda_{ \pm}$be the components of $(b, \phi)$ in the positive and negative spectral subspaces of $Q_{\alpha}$ respectively. Let $\left\|\lambda_{ \pm}\right\|$be the functions on $\left[R_{1}-1, R_{2}+1\right]$ given by taking the $L^{2}(Y)$-norm on 
the $t$-slice of $\left[R_{1}-1, R_{2}+1\right] \times Y$. Then the differential equation 26 gives rise to the following two differential inequalities

$$
\begin{gathered}
\partial_{t}\left\|\lambda_{+}\right\|-\left(\mu_{\alpha}-\epsilon\right)\left\|\lambda_{+}\right\|+\epsilon \lambda_{-} \| \geq 0 ; \\
\partial_{t}\left\|\lambda_{-}\right\|+\left(\mu_{\alpha}-\epsilon\right)\left\|\lambda_{-}\right\|-\epsilon \lambda_{+} \| \leq 0 .
\end{gathered}
$$

When $\epsilon<<\mu_{\alpha}$, the comparison principle (Cf. Lemma 9.4 in [32]) can be invoked to establish exponential decay with decay rate $\delta \leq \frac{\mu_{\alpha}-\epsilon}{2}$, that is, for any $t \in\left[R_{1}-1, R_{2}+1\right]$, the $L^{2}$-norm of $(b, \phi)$ on the $t$-slice of $\left[R_{1}-1, R_{2}+1\right] \times Y$ is bounded by $4 \epsilon\left(e^{-\delta\left(t-R_{1}\right)}+e^{-\delta\left(R_{2}-t\right)}\right)$. The standard bootstrapping argument of elliptic regularity theory implies that the $L_{1}^{2}$-distance from $[A(t), \psi(t)]$ for any $t \in\left[R_{1}, R_{2}\right]$ to the critical point $\alpha$ is bounded by $\epsilon\left(e^{-\delta\left(t-R_{1}\right)}+e^{-\delta\left(R_{2}-t\right)}\right)$, which is the claim (25).

Corollary 3.10. Let $(A(t), \psi(t))$ be a solution to the Seiberg-Witten equations (19) in temporal gauge with finite energy on $\mathbb{R} \times Y$, then there exist two 3-dimensional monopoles $\left(A_{\alpha}, \psi_{\alpha}\right)$ and $\left(A_{\beta}, \psi_{\beta}\right)$ representing two critical points $\alpha$ and $\beta$ in $\mathcal{M}_{Y}(\mathfrak{s}, \eta)$, such that

1. $A-A_{\alpha}$ and $\psi-\psi_{\alpha}$ decay exponentially along with their first derivatives as $t \rightarrow-\infty$,

2. $A-A_{\beta}$ and $\psi-\psi_{\beta}$ decay exponentially along with their first derivatives as $t \rightarrow \infty$.

Proof. To establish claim (a), we apply Lemma 3.9 to $(A(t), \psi(t))$ as a solution to the SeibergWitten equations $(19)$ in temporal gauge on $(-\infty,-R] \times Y$ with energy arbitrarily small for sufficiently large $R$. This gives the estimate (25) for $\left(A_{\alpha}, \psi_{\alpha}\right)$ representing a critical point $\alpha$ at any point $(t, y) \in(-\infty,-R] \times Y$ where, in (25) we take $R_{1}$ to infinity and $R_{2}=-R$. Claim (b) can be proved in the same way.

From this corollary, we know that if $(A(t), \psi(t))$ is a solution to the Seiberg-Witten equations (19) in temporal gauge with finite energy on $\mathbb{R} \times Y$, then there exists a sufficiently large $T$, such that for $t \in(-\infty,-T]$ or $t \in[T, \infty)$, the gauge equivalent class of $[A(t), \psi(t)]$ lies in a small $\epsilon$-neighborhood of some critical point $\alpha$ or $\beta$ respectively, and approaches the critical point exponentially fast.

\subsection{Moduli spaces of flowlines: transversality, compactification and orientibility.}

Let $\alpha, \beta$ be two critical points in $\mathcal{M}_{Y}(\mathfrak{s}, \eta)$. Denote by $\mathcal{M}(\alpha, \beta)$ the moduli space of solutions to the gradient flow equations (18) with the asymptotic limits $\alpha, \beta$ as $t \rightarrow-\infty,+\infty$. Note that there is an $\mathbb{R}$-action on $\mathcal{M}(\alpha, \beta)$ given by translation in the $t$ variable, $\mathbb{R}$ acts freely on $\mathcal{M}(\alpha, \beta)$ 
when $\alpha$ and $\beta$ are two different critical points. Denote by $\hat{\mathcal{M}}(\alpha, \beta)$ the quotient by this $\mathbb{R}$-action on $\mathcal{M}(\alpha, \beta)$.

Let $(A(t), \psi(t))$ represent a flowline in $\mathcal{M}(\alpha, \beta)$, then any flowline in the same component as $[A(t), \psi(t)]$ has constant energy, given by

$$
\mathcal{C}_{\eta}(A(-\infty), \psi(-\infty))-\mathcal{C}_{\eta}(A(\infty), \psi(\infty))
$$

where $(A(-\infty), \psi(-\infty))$ and $(A(\infty), \psi(\infty))$ are two asymptotic limits of $(A(t), \psi(t))$ as $t \rightarrow \pm \infty$, whose existence follows from Corollary 3.10 . There is a constant $\delta>0$ depending on the critical points, a constant $C$ depending only on the local geometry of $Y$ and perturbation $\eta$ such that for sufficiently large $T$

$$
\sum_{0 \leq k \leq 2}\left(\left|\nabla^{k}\left(A(t)-A_{ \pm \infty}\right)\right|+\mid\left(\nabla_{A_{ \pm \infty}}\right)^{k}(\psi(t)-\psi( \pm \infty) \mid) \leq C e^{-\delta(|t|-T)}\right.
$$

for $t \in(-\infty, T]$ and $t \in[T, \infty)$ respectively.

In this subsection, we will prove that generically each component of the moduli space $\mathcal{M}(\alpha, \beta)$ is a smooth manifold with dimension prescribed by the index theorem. We remind the reader that we always identify a path $(A(t), \psi(t))$ in $\mathcal{A}$ with a pair consisting of a $U(1)$-connection on the determinant bundle of the pull-back $\operatorname{Spin}^{c}$ structure and a spinor on $\pi^{*}(W)$ in temporal gauge.

To achieve transversality for the moduli space $\mathcal{M}(\alpha, \beta)$, we need to perturb the gradient flow equations in a non-local fashion:

$$
\left\{\begin{array}{l}
\frac{\partial A}{\partial t}=-* F_{A}+\sigma(\psi, \psi)+\eta(A, \psi) \\
\frac{\partial \psi}{\partial t}=-\not \partial_{A}(\psi)-\nu(A, \psi) \cdot \psi+\phi(A, \psi)
\end{array}\right.
$$

where $\eta(A, \psi), \nu(A, \psi)$ and $\phi(A, \psi)$ are functions of $(A, \psi)$. This general perturbation is chosen so as to preserve translation invariance of the gradient flow equation under the action of $\mathbb{R}$.

We now describe in detail how to construct such perturbations. For any Riemannian 3-manifold $(Y, g)$ with a $\operatorname{Spin}^{c}$ structure $\mathfrak{s}$, we choose a complete $L^{2}$-basis $\left\{\nu_{j}\right\}_{j=1}^{\infty}$ for the imaginary-valued 1-forms on $Y$, and a complete $L^{2}$-basis $\left\{\mu_{j}\right\}_{j=1}^{\infty}$ for the co-closed imaginaryvalued 1-forms on $Y$. Under the Hodge decomposition,

$$
\Omega_{L^{2}}^{1}(Y, i \mathbb{R})=H^{1}(Y, i \mathbb{R}) \oplus \operatorname{Im}\left(d^{*}\right) \oplus \operatorname{Im}(d)
$$

$\left\{\mu_{j}\right\}_{j=1}^{\infty}$ span the space $H^{1}(Y, i \mathbb{R}) \oplus \operatorname{Im}\left(d^{*}\right)$ and $\left\{\nu_{j}\right\}_{j=1}^{\infty}$ span the space $\Omega_{L^{2}}^{1}(Y, i \mathbb{R})$. We also need to choose a complete $L^{2}$-basis $\left\{\psi_{j}\right\}_{j=1}^{\infty}$ for the sections of the spinor bundle $W$. They are eigenvectors of the fixed Dirac operator $\not_{A_{0}}$ for a a fixed $U(1)$-connection $A_{0}$ on the determinant 
bundle $\operatorname{det}(\mathfrak{s})$. For each co-closed 1-form $\mu_{j}$, we associate a function on the configuration space $\mathcal{A}$ by

$$
\tau_{j}(A, \psi)=\tau_{j}\left(A-A_{0}\right)=\int_{Y}\left(A-A_{0}\right) \wedge * \mu_{j} .
$$

For simplicity, we assume that $\left\{\mu_{j}\right\}_{j=1}^{b_{1}}$ consist of a basis of $H^{1}(Y, i \mathbb{R})$, and $\left[* \mu_{j}\right]=0$ for $j>b_{1}$. It is easy to see that:

(1) $\tau_{j}$ is invariant under gauge transformations for $j>b_{1}$;

(2) for the map obtained from the first $b_{1}$ functions

$$
\left(\tau_{1}, \cdots, \tau_{b_{1}}\right): \mathcal{A} \rightarrow \mathbb{R}^{b_{1}},
$$

the gauge transformation $g: Y \rightarrow U(1)$ on $\mathcal{A}$ commutes with the $H^{1}(Y, \mathbb{Z}) \cong \mathbb{Z}^{b_{1}}$-action on $\mathbb{R}^{b_{1}}$ by translation by the vector

$$
<\left(\left[g^{-1} d g\right] \cup\left[* \mu_{1}\right],\left[g^{-1} d g\right] \cup\left[* \mu_{2}\right], \cdots,\left[g^{-1} d g\right] \cup\left[* \mu_{b_{1}}\right]\right),[Y]>.
$$

To each imaginary-valued 1 -form $\nu_{j}$ we associate a function $\zeta_{j}$ on $\mathcal{A}$ as follows

$$
\zeta_{j}(A, \psi)=\zeta_{j}(\psi, \psi)=\int_{Y}\left\langle\nu_{j} . \psi, \psi\right\rangle \operatorname{dvol}_{Y},
$$

where $\langle$,$\rangle is the Hermitian metric on the space of spinors. It is easy to see that \zeta_{j}$ is gauge invariant and real-valued. We also define $\zeta_{j}(\psi, \phi)=2 \int_{Y} \operatorname{Re}\left\langle\nu_{j} . \psi, \phi\right\rangle d v o l_{Y}$.

To each $\psi_{j}$, we associate a function $\xi_{j}$ on $\mathcal{A}$ as follows

$$
\xi_{j}(A, \psi)=\int_{Y}\left\langle e^{-\frac{1}{2} G d^{*}\left(A-A_{0}\right)} \psi_{j}, \psi\right\rangle d v o l_{Y},
$$

where $G$ is the Green's operator for the ordinary Laplacian on $L^{2}(Y)$. Let $\psi_{j}^{A}=e^{-\frac{1}{2} G d^{*}\left(A-A_{0}\right) \psi_{j}}$. Then $\xi_{j}$ is invariant under the action of the subgroup

$$
H=\left\{e^{i f} \mid f: Y \rightarrow \mathbb{R}, \int_{Y} f d v o l_{Y}=0\right\}
$$

of the identity component of the gauge group $\mathcal{G}_{Y}$ where $\mathcal{G}_{Y} / H \cong S^{1} \times H^{1}(Y, \mathbb{Z})$. The action of the $S^{1}$-factor has weight -1 , that is, for $u \in S^{1}$,

$$
\xi_{j}(u(A, \psi))=u^{-1} \xi_{j}(A, \psi) .
$$

For any $n \geq b_{1}(Y), k \geq 0$ and $l \geq 0,(\tau, \zeta, \xi)=\left(\tau_{1}, \cdots, \tau_{n}, \zeta_{1}, \cdots, \zeta_{k}, \xi_{1}, \cdots, \xi_{l}\right)$ defines a map $\mathcal{A} \rightarrow \mathbb{R}^{n} \times \mathbb{R}^{k} \times \mathbb{C}^{l}$ with the following key property.

Lemma 3.11. For any two points $\left(A_{1}, \psi_{1}\right)$ and $\left(A_{2}, \psi_{2}\right)$ in $\mathcal{A}$ representing two different points in $\mathcal{B}$, there exist $n, k$ and $l$ such that

$$
(\tau, \zeta, \xi)\left(A_{1}, \psi_{1}\right) \neq(\tau, \zeta, \xi)\left(A_{2}, \psi_{2}\right) .
$$


Proof. On the contrary, suppose that for any $n, k$ and $l,\left(\tau_{1}, \cdots, \tau_{n}, \zeta_{1}, \cdots, \zeta_{k}, \xi_{1}, \cdots, \xi_{l}\right)$ takes the same values on $\left(A_{1}, \psi_{1}\right)$ and $\left(A_{2}, \psi_{2}\right)$. Then from the definition of $\tau_{j}$, we know that $A_{1}=A_{2}-i d f$ for some real-valued function $f$ on $Y$, and from the definition of $\xi_{j}$, we know that

$$
e^{-\frac{1}{2} G d^{*}\left(A_{1}-A_{0}\right)} \psi_{1}=e^{-\frac{1}{2} G d^{*}\left(A_{2}-A_{0}\right)} \psi_{2},
$$

which implies that $\psi_{1}=e^{\frac{i}{2} f} \psi$. Hence $\left(A_{1}, \psi_{1}\right)$ and $\left(A_{2}, \psi_{2}\right)$ are gauge equivalent. This contradicts the assumption of the lemma.

As a corollary we have injectivity of the induced map

$$
(\tau, \zeta, \xi): \mathcal{B} \rightarrow \bigcup\left(\mathbb{R}^{n} \times \mathbb{R}^{k} \times \mathbb{C}^{l}\right) /\left(H^{1}(Y, \mathbb{Z}) \times S^{1}\right) s,
$$

which implies that there are sufficiently many perturbations to achieve the transversality of the moduli spaces of flowlines. This will be crucial in the proof of Proposition 3.16.

Now we choose any function $\mathfrak{p} \in C^{\infty}\left(\mathbb{R}^{n} \times \mathbb{R}^{k} \times \mathbb{C}^{l}, \mathbb{R}\right.$ ) (for $n \geq b_{1}, k, l>0$ ) where $\mathfrak{p}$ is invariant under the action of $H^{1}(Y, Z)$ on $\mathbb{R}^{b_{1}}$ (the first $b_{1}$-components of $\mathbb{R}^{N}$ ), and invariant under the action of $S^{1} \times H^{1}(Y, \mathbb{Z})$ on $\mathbb{C}^{l}$. We can define a function on $\mathcal{A} / \mathcal{G}$ by composition:

$$
\mathfrak{p}\left(\tau_{1}, \cdots, \tau_{n}, \zeta_{1}, \cdots, \zeta_{k}, \xi_{1}, \cdots, \xi_{l}\right)
$$

Let $\mathcal{C}_{\eta}$ be the Chern-Simons-Dirac function as in (14). We can perturb this functional to form

$$
\mathcal{C}_{\eta, \mathfrak{p}}=\mathcal{C}_{\eta}+\mathfrak{p}\left(\tau_{1}, \cdots, \tau_{n}, \zeta_{1}, \cdots, \zeta_{k}, \xi_{1}, \cdots, \xi_{l}\right)
$$

Let $\mathcal{P}$ be the subspace of $\bigcup_{n \geq b_{1}, k, l>0} C^{\infty}\left(\mathbb{R}^{n} \times \mathbb{R}^{k} \times \mathbb{C}^{l}, \mathbb{R}\right)$ which is invariant under the above action of $H^{1}(Y, \mathbb{Z}) \times S^{1} \times H^{1}(Y, \mathbb{Z})$ and has finite Floer $\epsilon$ norm. To be specific, choose $\underline{\epsilon}=\left(\epsilon_{k}\right)_{k \in \mathbb{N}}$ to be a given sequence of positive real numbers, then the Floer $\epsilon$-norm (cf. [9]): is given by

$$
\|\mathfrak{p}\|_{\underline{\epsilon}}=\sum_{k \geq 0} \epsilon_{k} \sup \left|\nabla^{k} \mathfrak{p}\right|
$$

By arguments similar to those in Lemma 5.1 of [9], the sequence $\underline{\epsilon}$ can be chosen such that $\mathcal{P}$ is a Banach space and $\mathcal{P}$ is dense in the subspace of $L_{k}^{2}$, whose elements are invariant under the above action of $H^{1}(Y, \mathbb{Z}) \times S^{1} \times H^{1}(Y, \mathbb{Z})$.

For each $\mathfrak{p} \in \mathcal{P}$, the gradient of $\mathcal{C}_{\eta, \mathfrak{p}}$ at $[A, \psi]$ with respect to the natural $L^{2}$ inner product is given by

$$
\begin{gathered}
\left(* F_{A}-\sigma(\psi, \psi)-\eta-\sum_{j=1}^{n} \frac{\partial \mathfrak{p}}{\partial \tau_{j}} \mu_{j}+\sum_{j=1}^{l} \frac{\partial \mathfrak{p}}{\partial \xi_{j}} d \circ G\left(i \operatorname{Im}<\psi_{j}^{A}, \psi>\right),\right. \\
\left.\partial_{A}(\psi)+\sum_{j=1}^{K} \frac{\partial \mathfrak{p}}{\partial \zeta_{j}} \nu_{j} \cdot \psi+\sum_{j=1}^{l} \frac{\partial \mathfrak{p}}{\partial \xi_{j}} \psi_{j}^{A}\right) .
\end{gathered}
$$


Here $\nabla \mathfrak{p}=\left(-\sum_{j=1}^{n} \frac{\partial \mathfrak{p}}{\partial \tau_{j}} \mu_{j}+\sum_{j=1}^{l} \frac{\partial \mathfrak{p}}{\partial \xi_{j}} d \circ G\left(i \operatorname{Im}<\psi_{j}^{A}, \psi>\right), \sum_{j=1}^{K} \frac{\partial \mathfrak{p}}{\partial \zeta_{j}} \nu_{j} . \psi+\sum_{j=1}^{l} \frac{\partial \mathfrak{p}}{\partial \xi_{j}} \psi_{j}^{A}\right)$.

In order not to change the critical points of $\mathcal{C}_{\eta}$ when perturbed by $\mathfrak{p}$, we need to impose some condition on $\mathfrak{p} \in \mathcal{P}$. We remind the reader that, as in Lemma 3.3, given a small $\epsilon>0$, there is a constant $\lambda$, such that

$$
\left\|\nabla \mathcal{C}_{\eta}([A, \psi])\right\|_{L^{2}}=\left\|\left(* F_{A}-\sigma(\psi, \psi)-\eta, \not \partial_{A}(\psi)\right)\right\|_{L^{2}}>\lambda
$$

for $[A, \psi]$ at $L_{1}^{2}$-distance at least $\epsilon$ away from the set of critical points. For each critical point $\alpha \in \mathcal{M}_{Y}(\mathfrak{s}, \eta)$, let $B(\alpha, \epsilon)$ be ball of radius $\epsilon$ centered at $\alpha$ in the $L_{1}^{2}$ topology.

Condition 3.12. We require that the perturbation function $\mathfrak{p} \in \mathcal{P}$ satisfies the following conditions:

1. $\mathfrak{p}=0$ on $\bigcup_{\alpha \in \mathcal{M}_{Y}(\mathfrak{s}, \eta)}(\tau, \zeta, \tau)(B(\alpha, \epsilon))$.

2. The corresponding perturbation term $\nabla \mathfrak{p}$ has $L^{2}$-norm less than $\epsilon_{0} \lambda$ for a sufficiently small $\epsilon_{0}<<1$.

Let $\mathcal{P}_{c}$ be the set of perturbation functions $\mathfrak{p} \in \mathcal{P}$ satisfying (a) and (b).

The corresponding downward gradient flow equation of $\mathcal{C}_{\mathfrak{p}}$ is given by

$$
\left\{\begin{aligned}
\frac{\partial A}{\partial t} & =-* F_{A}+\sigma(\psi, \psi)+\eta+\sum_{j=1}^{N} \frac{\partial \mathfrak{p}}{\partial \tau_{j}} \mu_{j}-\sum_{j=1}^{l} \frac{\partial \mathfrak{p}}{\partial \xi_{j}} d \circ G\left(i \operatorname{Im}<\psi_{j}^{A}, \psi>\right) \\
\frac{\partial \psi}{\partial t} & =-\not_{A} \psi-\sum_{j=1}^{K} \frac{\partial \mathfrak{p}}{\partial \zeta_{j}} \nu_{j} . \psi-\sum_{j=1}^{l} \frac{\partial \mathfrak{p}}{\partial \xi_{j}} \psi_{j}^{A}
\end{aligned}\right.
$$

for a pair $(A(t), \Phi(t))$ regarded as a family of connections and spinors. These are the perturbed 4-dimensional Seiberg-Witten equations on $Y \times \mathbb{R}$ in temporal gauge. The corresponding perturbation of the Seiberg-Witten equations on $Y \times \mathbb{R}$ is given by

$$
\left\{\begin{array}{l}
F_{A}^{+}=q(\psi, \psi)+\eta^{+}+\sum_{j=1}^{N} \frac{\partial \mathfrak{p}}{\partial \tau_{j}} \mu_{j}^{+}-\sum_{j=1}^{l} \frac{\partial \mathfrak{p}}{\partial \xi_{j}}\left(d \circ G\left(i \operatorname{Im}<\psi_{j}^{A}, \psi>\right)\right)^{+} \\
\not D_{A}(\psi)+\sum_{j=1}^{K} \frac{\partial \mathfrak{p}}{\partial \zeta_{j}} \nu_{j} \cdot \psi+\sum_{j=1}^{l} \frac{\partial \mathfrak{p}}{\partial \xi_{j}} \psi_{j}^{A}=0
\end{array}\right.
$$

where $\mu_{j}^{+}=\frac{1}{2}\left(*_{3} \mu_{j}+\mu_{j} \wedge d t\right)$ and $\left(d \circ G\left(i \operatorname{Im}<\psi_{j}^{A}, \psi>\right)\right)^{+}$is the self-dual part of $d \circ G\left(i \operatorname{Im}<\psi_{j}^{A}, \psi>\right) \wedge d t$, and the function $\mathfrak{p}$ is the corresponding function on the 4-dimensional configuration space of $U(1)$-connections and spinors on $\left(\mathbb{R} \times Y d t^{2}+g\right)$ with the pull back $\operatorname{Spin}^{c}$ structure. Note that $\mathfrak{p}$ is invariant under gauge transformations. It is easy to see that $\mathfrak{p}$ is a well-defined function over the 4-dimensional configuration space. For any solution $(A(t), \psi(t))$ to the perturbed Seiberg-Witten equations (30) on $\left[R_{1}, R_{2}\right] \times Y$ in temporal gauge, we say that 
$(A(t), \psi(t))$ has finite energy if and only if

$$
\int_{R_{1}}^{R_{2}}\left(\left\|\frac{\partial A(t)}{\partial t}\right\|_{L^{2}(Y)}^{2}+\left\|\frac{\partial \psi(t)}{\partial t}\right\|_{L^{2}(Y)}^{2}\right) d t<\infty .
$$

Lemma 3.13. Let $\gamma(t)=(A(t), \psi(t))$ be a solution to the perturbed Seiberg-Witten equations (30) on $\left[R_{1}, R_{2}\right] \times Y$ in temporal gauge, then $(A(t), \psi(t))$ has finite energy if and only if one of the following terms is finite:

1. $\mathcal{C}_{\eta}\left(A\left(R_{1}\right), \psi\left(R_{1}\right)\right)-\mathcal{C}_{\eta}\left(A\left(R_{2}\right), \psi\left(R_{2}\right)\right)$,

2. $\mathcal{C}_{\eta, \mathfrak{p}}\left(A\left(R_{1}\right), \psi\left(R_{1}\right)\right)-\mathcal{C}_{\eta, \mathfrak{p}}\left(A\left(R_{2}\right), \psi\left(R_{2}\right)\right)$,

3. $\mathcal{C}\left(A\left(R_{1}\right), \psi\left(R_{1}\right)\right)-\mathcal{C}\left(A\left(R_{2}\right), \psi\left(R_{2}\right)\right)$,

4. $E_{\left[R_{1}, R_{2}\right] \times Y}(\mathbb{A}, \Psi)$ (as defined in (21)), where $(\mathbb{A}, \Psi)=(A(t), \psi(t))$ is the corresponding 4-dimensional monopole on $\left[R_{1}, R_{2}\right] \times Y$.

Proof. Note that $\gamma(t)=(A(t), \psi(t))$ satisfies the perturbed Seiberg-Witten equations (30) on $\left[R_{1}, R_{2}\right] \times Y$ in temporal gauge, that is, $\frac{\partial \gamma(t)}{\partial t}=-\nabla \mathcal{C}_{\eta, \mathfrak{p}}(\gamma(t))$, hence we have

$$
\begin{aligned}
& \mathcal{C}_{\eta}\left(A\left(R_{1}\right), \psi\left(R_{1}\right)\right)-\mathcal{C}_{\eta}\left(A\left(R_{2}\right), \psi\left(R_{2}\right)\right) \\
= & -\int_{R_{1}}^{R_{2}}<\nabla \mathcal{C}_{\eta}(\gamma(t)), \frac{\partial \gamma(t)}{\partial t}>d t \\
= & \int_{R_{1}}^{R_{2}}<\nabla \mathcal{C}_{\eta}(\gamma(t)), \nabla \mathcal{C}_{\eta, \mathfrak{p}}(\gamma(t))>d t \\
= & \int_{R_{1}}^{R_{2}}\left(\left\|\nabla \mathcal{C}_{\eta}(\gamma(t))\right\|_{L^{2}}^{2}+<\nabla \mathcal{C}_{\eta}(\gamma(t)), \nabla \mathfrak{p}(\gamma(t))>\right) d t .
\end{aligned}
$$

and

$$
\begin{aligned}
& \mathcal{C}_{\eta, \mathfrak{p}}\left(A\left(R_{1}\right), \psi\left(R_{1}\right)\right)-\mathcal{C}_{\eta, \mathfrak{p}}\left(A\left(R_{2}\right), \psi\left(R_{2}\right)\right) \\
= & -\int_{R_{1}}^{R_{2}}<\nabla \mathcal{C}_{\eta, \mathfrak{p}}(\gamma(t)), \frac{\partial \gamma(t)}{\partial t}>d t \\
= & \int_{R_{1}}^{R_{2}}\left\|\frac{\partial \gamma(t)}{\partial t}\right\|_{L^{2}}^{2} d t \\
= & \int_{R_{1}}^{R_{2}}\left\|\nabla \mathcal{C}_{\eta, \mathfrak{p}}(\gamma(t))\right\|_{L^{2}}^{2} d t .
\end{aligned}
$$

From Condition 3.12, we get

$$
\left\|\nabla \mathcal{C}_{\eta}(\gamma(t))-\nabla \mathcal{C}_{\eta, \mathfrak{p}}(\gamma(t))\right\|_{L^{2}}<\epsilon_{0}\left\|\mathcal{C}_{\eta}(\gamma(t))\right\|_{L^{2}} .
$$

Putting all these together and setting $\epsilon=\epsilon_{0} / 5$, we have

$$
1-\epsilon<\frac{\mathcal{C}_{\eta}\left(A\left(R_{1}\right), \psi\left(R_{1}\right)\right)-\mathcal{C}_{\eta}\left(A\left(R_{2}\right), \psi\left(R_{2}\right)\right.}{\mathcal{C}_{\eta, \mathfrak{p}}\left(A\left(R_{1}\right), \psi\left(R_{1}\right)\right)-\mathcal{C}_{\eta, \mathfrak{p}}\left(A\left(R_{2}\right), \psi\left(R_{2}\right)\right)}<1+\epsilon
$$


Similarly, under the condition that $\eta$ is sufficiently small as in Remark 3.4, we can prove that

$$
1-\epsilon<\frac{\mathcal{C}_{\eta}\left(A\left(R_{1}\right), \psi\left(R_{1}\right)\right)-\mathcal{C}_{\eta}\left(A\left(R_{2}\right), \psi\left(R_{2}\right)\right.}{\mathcal{C}\left(A\left(R_{1}\right), \psi\left(R_{1}\right)\right)-\mathcal{C}\left(A\left(R_{2}\right), \psi\left(R_{2}\right)\right)}<1+\epsilon,
$$

These last two inequalities imply that $\gamma(t)$ has finite energy if and only if any one of $\mathcal{C}_{\eta}\left(\gamma\left(R_{1}\right)\right)-\mathcal{C}_{\eta}\left(\gamma\left(R_{2}\right)\right), \mathcal{C}\left(A\left(R_{1}\right), \psi\left(R_{1}\right)\right)-\mathcal{C}\left(A\left(R_{2}\right), \psi\left(R_{2}\right)\right)$ or $\mathcal{C}_{\eta, \mathfrak{p}}\left(\gamma\left(R_{1}\right)\right)-\mathcal{C}_{\eta, \mathfrak{p}}\left(\gamma\left(R_{2}\right)\right)$ is finite.

For the proof of part (4), direct calculation leads to the following identities:

$$
\begin{aligned}
& \mathcal{C}_{\eta}\left(A\left(R_{1}\right), \psi\left(R_{1}\right)\right)-\mathcal{C}_{\eta}\left(A\left(R_{2}\right), \psi\left(R_{2}\right)\right) \\
= & \frac{1}{2} \int_{\left[R_{1}, R_{2}\right] \times Y}\left(F_{\mathbb{A}}-* \eta\right) \wedge\left(F_{\mathbb{A}}-* \eta\right) \\
& +\int_{\left\{R_{1}\right\} \times Y}<\psi, \not_{A} \psi>d v o l_{Y}-\int_{\left\{R_{2}\right\} \times Y}<\psi, \not_{A} \psi>d v o l_{Y} .
\end{aligned}
$$

and

$$
\begin{aligned}
& \mathcal{C}\left(A\left(R_{1}\right), \psi\left(R_{1}\right)\right)-\mathcal{C}\left(A\left(R_{2}\right), \psi\left(R_{2}\right)\right) \\
= & \frac{1}{2} \int_{\left[R_{1}, R_{2}\right] \times Y} F_{\mathbb{A}} \wedge F_{\mathbb{A}} \\
& +\int_{\left\{R_{1}\right\} \times Y}<\psi, \not \partial_{A} \psi>d v o l_{Y}-\int_{\left\{R_{2}\right\} \times Y}<\psi, \not \partial_{A} \psi>d v o l_{Y} .
\end{aligned}
$$

To prove the last claim, we write the perturbed Seiberg-Witten equations (30) as follows:

$$
\left\{\begin{array}{l}
F_{\mathbb{A}}^{+}=q(\Psi, \Psi)+\mu^{+}+\mathfrak{p}_{\mathbb{A}}^{+} \\
\not D_{\mathbb{A}}(\Psi)+\mathfrak{p}_{\Psi}=0 .
\end{array}\right.
$$

where $q(\Psi, \Psi)=(d t \wedge \sigma(\psi, \psi))^{+}$and $\left(\mathfrak{p}_{\mathbb{A}}^{+}, \mathfrak{p}_{\Psi}\right)$ is the perturbation term coming from the perturbation $\mathfrak{p}$ (see (30) for exact expression), notice that, by Condition 3.12, the $L^{2}$-norm of $\left(\mathfrak{p}_{A}^{+}, \mathfrak{p}_{\psi}\right)$ is less than $\epsilon_{0}\left\|\nabla \mathcal{C}_{\eta}\right\|_{L^{2}}$ for a sufficiently small $\epsilon_{0}<<1$.

Now we calculate

$$
\int_{\left[R_{1}, R_{2}\right] \times Y}\left(\left|\not D_{\mathbb{A}}(\Psi)\right|^{2}+\left|F_{\mathbb{A}}^{+}-q(\Psi, \Psi)-\mu^{+}\right|^{2}\right) d t d v o l_{Y} .
$$

using the 3- and 4-dimensional Weitzenböck formulae

The 4-dimensional Weitzenböck formula is

$$
\not D_{\mathbb{A}}^{*} D_{\mathbb{A}}(\Psi)=\nabla_{\mathbb{A}}^{*} \nabla_{\mathbb{A}} \Psi+\frac{s}{4} \Psi+\frac{1}{2} F_{\mathbb{A}}^{+} \cdot \Psi,
$$

where $s$ denotes the scalar curvature on $\left(\mathbb{R} \times Y, g_{Y}+d t^{2}\right)$. Take the inner product of both sides with $\Psi=\psi(t)$, and then integrate them over $\left[R_{1}, R_{2}\right] \times Y$. Here we need to understand the boundary contribution when we integrate by parts. To see what this contribution is, we proceed 
as follows

$$
\begin{aligned}
& \int_{\left[R_{1}, R_{2}\right] \times Y}\left|\not D_{\mathbb{A}} \Psi\right|^{2} d t d v o l_{Y} \\
= & \int_{\left[R_{1}, R_{2}\right] \times Y}\left(\left|\frac{\partial \psi(t)}{\partial t}+\not \partial_{A} \psi\right|^{2}\right) d t d v o l_{Y} \\
= & \int_{\left[R_{1}, R_{2}\right] \times Y}\left(\left|\frac{\partial \psi(t)}{\partial t}\right|^{2}+\left|\not_{A} \psi\right|^{2}+<\frac{\partial \psi(t)}{\partial t}, \not_{A} \psi>+<\not \partial_{A} \psi, \frac{\partial \psi(t)}{\partial t}\right) d t d v o l_{Y} \\
= & \int_{\left[R_{1}, R_{2}\right] \times Y}\left(\left|\frac{\partial \psi(t)}{\partial t}\right|^{2}+\left|\not_{A} \psi\right|^{2}+\frac{\partial}{\partial t}\left(<\psi, \not_{A} \psi>\right)-\frac{1}{2}<\frac{\partial A(t)}{\partial t} \cdot \psi, \psi>\right) d t d v o l_{Y} .
\end{aligned}
$$

Now apply the 3-dimensional Weitzenböck formula,

$$
\not \partial_{A}^{2} \psi=\nabla_{A}^{*} \nabla_{A} \psi+\frac{s}{4} \psi-\frac{1}{2}\left(* F_{A}\right) \cdot \psi,
$$

and note that $F_{\mathbb{A}}^{+} \cdot \Psi=\left(\frac{\partial A(t)}{\partial t}+*_{3} F_{A}\right) . \psi$, where $*_{3}$ is the Hodge star operator on $Y$. Using the various expressions above, we obtain that

$$
\begin{aligned}
& \int_{\left[R_{1}, R_{2}\right] \times Y}\left|\not D_{\mathbb{A}}(\Psi)\right|^{2} d t d v o l_{Y} \\
= & \int_{\left[R_{1}, R_{2}\right] \times Y}\left(\left|\nabla_{\mathbb{A}} \Psi\right|^{2}+\frac{s}{4}|\Psi|^{2}-\frac{1}{2}<F_{\mathbb{A}}^{+} . \Psi, \Psi>\right) d t d v o l_{Y} \\
& +\int_{\left\{R_{2}\right\} \times Y}<\psi, \not \partial_{A} \psi>d v o l_{Y}-\int_{\left\{R_{1}\right\} \times Y}<\psi, \partial_{A} \psi>d v o l_{Y} .
\end{aligned}
$$

We also have

$$
\begin{aligned}
& \int_{\left[R_{1}, R_{2}\right] \times Y} 2\left|F_{\mathbb{A}}^{+}-q(\Psi, \Psi)-\eta^{+}\right|^{2} d t d v o l_{Y} \\
= & \int_{\left[R_{1}, R_{2}\right] \times Y}\left(\left(\left|F_{\mathbb{A}}\right|^{2}+|\sigma(\psi, \psi)+\eta|^{2}+<F_{\mathbb{A}}^{+} \cdot \Psi, \Psi>\right) d t d v o l_{Y}\right. \\
& -\left(F_{\mathbb{A}}-*_{3} \eta\right) \wedge\left(F_{\mathbb{A}}-*_{3} \eta\right)+2 d t \wedge F_{\mathbb{A}} \wedge \eta .
\end{aligned}
$$

Using the perturbed Seiberg-Witten equations (35) and the identity (33), we have

$$
\begin{aligned}
& \int_{\left[R_{1}, R_{2}\right] \times Y} 2\left(\left|\left(\mathfrak{p}_{\mathbb{A}}^{+}, \mathfrak{p}_{\Psi}\right)\right|^{2}\right) d t d v o l_{Y} \\
= & \int_{\left[R_{1}, R_{2}\right] \times Y}\left(2\left|\not D_{\mathbb{A}}(\Psi)\right|^{2}+2\left|F_{\mathbb{A}}^{+}-q(\Psi, \Psi)-\eta^{+}\right|^{2}\right) d t d v o l_{Y} \\
= & \int_{\left[t_{1}, t_{2}\right] \times Y}\left(\left|F_{\mathbb{A}}\right|^{2}+2\left|q(\Psi, \Psi)+\eta^{+}\right|^{2}+2\left|\nabla_{\mathbb{A}} \Psi\right|^{2}+\frac{s}{2}|\Psi|^{2}\right) d t d v o l_{Y} \\
& +\int_{\left[t_{1}, t_{2}\right] \times Y}\left(2\left(F_{\mathbb{A}} \wedge d t \wedge \eta\right)-\left(F_{\mathbb{A}}-*_{3} \eta\right) \wedge\left(F_{\mathbb{A}}-*_{3} \eta\right)\right) \\
& +2\left(\int_{\left\{R_{2}\right\} \times Y}<\psi, \not \partial_{A} \psi>d v o l_{Y}-\int_{\left\{R_{1}\right\} \times Y}<\psi, \not_{A} \psi>d v o l_{Y}\right) \\
= & E_{\left[R_{1}, R_{2}\right] \times Y}(\mathbb{A}, \Psi)+2\left(\mathcal{C}_{\eta}\left(A\left(R_{2}\right), \psi\left(R_{2}\right)\right)-\mathcal{C}_{\eta}\left(A\left(R_{1}\right), \psi\left(R_{1}\right)\right)\right) .
\end{aligned}
$$

Thus, we have the following inequalities $\left(\epsilon=\epsilon_{0} / 5\right)$

$$
2<\frac{E_{\left[R_{1}, R_{2}\right] \times Y}(\mathbb{A}, \Psi)}{\mathcal{C}_{\eta}\left(A\left(R_{1}\right), \psi\left(R_{1}\right)\right)-\mathcal{C}_{\eta}\left(A\left(R_{2}\right), \psi\left(R_{2}\right)\right)}<2+\epsilon
$$


which implies that $\mathcal{C}_{\eta}\left(A\left(R_{1}\right), \psi\left(R_{1}\right)\right)-\mathcal{C}_{\eta}\left(A\left(R_{2}\right), \psi\left(R_{2}\right)\right)$ is finite if and only if $E_{\left[R_{1}, R_{2}\right] \times Y}(\mathbb{A}, \Psi)$ is finite. The completes the proof of the Lemma.

The following lemma states the basic properties of the perturbed CSD functional $\mathcal{C}_{\eta, \mathfrak{p}}$. The proof follows the same arguments as are used for the corresponding properties of the CSD functional $\mathcal{C}_{\eta}$ and of the perturbation terms satisfying Condition 3.12 for $\mathfrak{p} \in \mathcal{P}_{c}$.

Lemma 3.14. For any $\mathfrak{p} \in \mathcal{P}_{c}$, the perturbed Chern-Simons-Dirac functional $\mathcal{C}_{\eta, \mathfrak{p}}$ on $\mathcal{B}$ has the following properties:

1. The critical point set of $\mathcal{C}_{\eta, \mathfrak{p}}$ agrees with the critical point set of $\mathcal{C}_{\eta}$, and all points are non-degenerate.

2. The Hessian operator of $\mathcal{C}_{\eta, \mathfrak{p}}$ on $\mathcal{B}$ is a compact perturbation of the Hessian operator of $\mathcal{C}_{\eta}$, hence has the same properties as the Hessian operator of $\mathcal{C}_{\eta}$ in Lemma 3.1.

3. Let $(A(t), \psi(t))$ be a solution to the perturbed Seiberg-Witten equations (30) on $\mathbb{R} \times Y$ in temporal gauge with finite energy, then there exists a sufficiently large $T$ such that on $(-\infty,-T] \times Y$ and $[T, \infty) \times Y$ the perturbation term from $\mathfrak{p}$ is zero, in other words, $\mathfrak{p}(\tau, \zeta, \xi)(A(t), \psi(t))$ has compact support in $[-T, T]$. Hence, $(A(t), \psi(t))$ is a solution to the gradient flow equation $(18)$ of $\mathcal{C}_{\eta}$ outside the compact set $[-T, T] \times Y$.

4. There is a constant $\delta>0$, such that a choice of sufficiently small $\epsilon$ determines $E_{0}>0$ with the following property: suppose that $(A(t), \psi(t))$ is a solution to the perturbed SeibergWitten equations (30) on $\left[R_{1}-2, R_{2}+2\right] \times Y$ in temporal gauge with energy

$$
E_{\left[R_{1}-2, R_{2}+2\right] \times Y}(A(t), \psi(t))<E_{0},
$$

then there exists a critical point $\alpha \in \mathcal{M}_{Y}(\mathfrak{s}, \eta)$ such that the $L_{1}^{2}$-distance from $[A(t), \psi(t)]$ (for any $\left.t \in\left[R_{1}, R_{2}\right]\right)$ to $\alpha$ is at most $4 \epsilon\left(e^{-\delta\left(t-R_{1}\right)}+e^{-\delta\left(R_{2}-t\right)}\right)$. Moreover, there exists a sequence of constants $\left\{\lambda_{k}\right\}_{k=0,1,2, \cdots}$ such that there is a 3-dimensional monopole $\left(A_{\alpha}, \psi_{\alpha}\right)$ representing $\alpha$ for which

$$
\left|\nabla^{k}\left(A(t)-A_{\alpha}\right)\right|+\left|\left(\nabla_{A_{\alpha}}\right)^{k}\left(\psi(t)-\psi_{\alpha}\right)\right| \leq \lambda_{k}\left(e^{-\delta\left(t-R_{1}\right)}+e^{-\delta\left(R_{2}-t\right)}\right),
$$

for any point $(t, y) \in\left[R_{1}, R_{2}\right] \times Y$.

5. Let $(A(t), \psi(t))$ be a solution to the perturbed Seiberg-Witten equations (30) on $\mathbb{R} \times Y$ in temporal gauge with finite energy, then there exist two 3-dimensional monopoles $\left(A_{\alpha}, \psi_{\alpha}\right)$ and $\left(A_{\beta}, \psi_{\beta}\right)$ representing two critical points $\alpha$ and $\beta$ in $\mathcal{M}_{Y}(\mathfrak{s}, \eta)$, such that $A-A_{\alpha}$ and $\psi-\psi_{\alpha}$ decay exponentially along with their first derivatives as $t \rightarrow-\infty$, and $A-A_{\beta}$ and $\psi-\psi_{\beta}$ decay exponentially along with their first derivatives as $t \rightarrow \infty$. 
Corollary 3.15. If $(A(t), \psi(t))$ is a solution to the perturbed Seiberg-Witten equations (39) on $[0, \infty) \times Y$ in temporal gauge with finite energy, then there exists a sequence of constants $\left\{\lambda_{k}\right\}_{k=0,1,2, \ldots}$ such that there is a 3-dimensional monopole $\left(A_{\alpha}, \psi_{\alpha}\right)$ representing a critical point $\alpha$ in $\mathcal{M}_{Y}(\mathfrak{s}, \eta)$ for which

$$
\left|\nabla^{k}\left(A(t)-A_{\alpha}\right)\right|+\left|\left(\nabla_{A_{\alpha}}\right)^{k}\left(\psi(t)-\psi_{\alpha}\right)\right| \leq \lambda_{k} e^{-\delta t},
$$

for any point $(t, y) \in[0, \infty) \times Y$, and

$$
\left|\int_{[0, \infty) \times Y} F_{\mathbb{A}} \wedge F_{\mathbb{A}}\right|<\infty .
$$

We now study the geometric structure of the moduli space $\mathcal{M}_{\mathfrak{p}}(\alpha, \beta)$ for the gradient flow lines of $\mathcal{C}_{\eta, \mathfrak{p}}$ on $\mathcal{B}$ connecting two different critical points $\alpha, \beta$ of $\mathcal{M}_{Y}(\mathfrak{s}, \eta)$. The space $\mathcal{M}_{\mathfrak{p}}(\alpha, \beta)$ is also the moduli space of solutions to the perturbed Seiberg-Witten equations on $\left(Y \times \mathbb{R}, g+d t^{2}, \pi^{*}(\mathfrak{s})\right)$, whose asymptotic limits at $\pm \infty$ represent $\alpha$ and $\beta$ respectively. Let $\delta$ be the decay rate of the gradient flow line in $\mathcal{M}_{\mathfrak{p}}(\alpha, \beta)$ (see Proposition 3.9 and Lemma 3.14).

We will consider the space of pairs consisting of $U(1)$ connections on the determinant bundle and sections of the spinor bundle on

$$
\left(Y \times \mathbb{R}, g+d t^{2}, \pi^{*}(\mathfrak{s})\right)
$$

topologized with weighted Sobolev norms of weight $\delta$ as in [18]. Here the weight function is $e_{\delta}(t)=e^{\tilde{\delta} t}$, where $\tilde{\delta}$ is a smooth function with bounded derivatives, $\tilde{\delta}: \mathbb{R} \rightarrow[-\delta, \delta]$ such that $\tilde{\delta}(t) \equiv-\delta$ for $t \leq-1$ and $\tilde{\delta}(t) \equiv \delta$ for $t \geq 1$. The $L_{k, \delta}^{2}$ norm is defined as

$$
\|f\|_{L_{k, \delta}^{2}}=\left(\int_{Y \times \mathbb{R}} e_{\delta}(t)\left(|f|^{2}+|\nabla f|^{2}+\left|\nabla^{2} f\right|^{2}+\cdots+\left|\nabla^{k} f\right|^{2}\right) d t d v o l_{Y}\right)^{\frac{1}{2}}
$$

The weight $e_{\delta}$ imposes an exponential decay as an asymptotic condition along the cylinder.

An element $\gamma_{0}$ of $\mathcal{M}(\alpha, \beta)$ determines a path in $\mathcal{A} / \mathcal{G}$, also denoted $\gamma_{0}$. We lift $\gamma_{0}$ to a path in $\mathcal{A}$ which we denote by $\tilde{\gamma}_{0}=\left(A_{0}(t), \psi_{0}(t)\right)$. From Lemma 3.14, we know that $\left(A_{\alpha}, \psi_{\alpha}\right)$ and $\left(A_{\beta}, \psi_{\beta}\right)$ represent $\alpha$ and $\beta$ respectively, such that $\left(A_{0}(t), \psi_{0}(t)\right)$ approaches to $\left(A_{\alpha}, \psi_{\alpha}\right)$ and $\left(A_{\beta}, \psi_{\beta}\right)$ exponentially fast in the $L_{1}^{2}$-topology as $t \rightarrow \pm \infty$ respectively.

The operators $T(t)$ have a spectral flow denoted by $\mathfrak{i}\left(\gamma_{0}\right)$ along the path $\tilde{\gamma}_{0}$ (see (16) for the relation with the spectral flow of the Hessian operator $Q$ ). We see that

$$
\mathfrak{i}\left(\gamma_{0}\right)=S F_{\gamma_{0}}(Q)=\mathfrak{i}(\alpha, \beta)(\bmod d(\mathfrak{s}))
$$

where $\mathfrak{i}(\alpha, \beta)$ is the relative index between $\alpha$ and $\beta$, defined in (3.6). Note that $\mathfrak{i}\left(\gamma_{0}\right)$ is $\mathbb{Z}$-valued.

Choose a $U(1)$-connection $\mathbb{A}_{0}$ on $\operatorname{det}\left(W^{+}\right)$and a spinor section $\Psi_{0}$ of $W^{+}$such that $\left(A_{0}, \Psi_{0}\right)$ is in temporal gauge outside a compact set and satisfies $\left(\mathbb{A}_{0}, \Psi_{0}\right)=\left(A_{\alpha}, \psi_{\alpha}\right)$ for $t<<0$ and $\left(\mathbb{A}_{0}, \Psi_{0}\right)=\left(A_{\beta}, \psi_{\beta}\right)$ for $t>>0$. 
For $k \geq 2$, let $\mathcal{A}_{k, \delta}(\alpha, \beta)$ be the space of pairs of connections and spinor sections $(A, \psi)$ on $Y \times \mathbb{R}$ satisfying

$$
(A, \psi) \in\left(\mathbb{A}_{0}, \Psi_{0}\right)+\left(\Omega_{L_{k, \delta}^{2}}^{1}(Y \times \mathbb{R}, i \mathbb{R}) \oplus L_{k, \delta}^{2}\left(W^{+}\right)\right) .
$$

The gauge transformation group $\mathcal{G}_{k+1, \delta}(\alpha, \beta)$ is locally modelled on

$$
L_{k+1, \delta}^{2}\left(\Omega^{0}(Y \times \mathbb{R}, i \mathbb{R})\right)
$$

and approaches elements in the stabilizers $G_{\alpha}$ and $G_{\beta}$ of $\alpha$ and $\beta$ as $t \rightarrow \pm \infty$. This gauge group acts on $\mathcal{A}_{k, \delta}(\alpha, \beta)$ freely, so we can form the quotient $\mathcal{B}_{k, \delta}(\alpha, \beta)$, a smooth Banach manifold, whose tangent space at $[A, \psi]$ (represented by $\left.(A, \psi) \in \mathcal{A}_{k, \delta}(\alpha, \beta)\right)$ is given by

$$
\left\{(a, \phi) \in \Omega_{L_{k, \delta}^{2}}^{1} \oplus L_{k, \delta}^{2}\left(W^{+}\right) \mid G_{[A, \psi], \delta}^{*}(a, \phi)=\left(e_{-\delta} d^{*} e_{\delta}\right) a+i \operatorname{Im}\langle\psi, \phi\rangle=0\right\} .
$$

Using Proposition 3.9, we know that the component of $\mathcal{M}_{\mathfrak{p}}(\alpha, \beta)$ containing the given gradient flow line $\gamma_{0}$, denoted by $\mathcal{M}_{\mathfrak{p}}(\alpha, \beta)_{\gamma_{0}}$, can be identified with

$$
\left\{\begin{array}{l|l}
(A, \psi) \in \mathcal{A}_{k, \delta}(\alpha, \beta) & \begin{array}{l}
(A, \psi) \text { satisfies the } \\
\text { monopole equations (30). }
\end{array}
\end{array}\right\} / \mathcal{G}_{k+1, \delta}(\alpha, \beta) .
$$

Proposition 3.16. There is a Baire set $\mathcal{P}_{0}$ of smooth functions $\mathfrak{p} \in \mathcal{P}_{c}$, satisfying Condition 3.17, such that for $\mathfrak{p} \in \mathcal{P}_{0} \mathcal{M}_{\mathfrak{p}}(\alpha, \beta)_{\gamma_{0}}$, the component of $\mathcal{M}(\alpha, \beta)$ containing $\gamma_{0}$, if non-empty, is a smooth oriented manifold of dimension $\mathfrak{i}\left(\gamma_{0}\right)-d_{\alpha}$ where $d_{\alpha}=1$ if $\alpha$ is reducible and $d_{\alpha}=0$ otherwise.

Proof. Suppose $(A(t), \psi(t), \mathfrak{p})$, representing a point in $\mathcal{M}_{\mathfrak{p}}(\alpha, \beta)_{\gamma_{0}}$, is a solution to the perturbed gradient flow equation (27) or the perturbed Seiberg-Witten equations (30) in temporal gauge with the perturbation $\mathfrak{p} \in \mathcal{P}_{c}$. Using the linearization of (30) and gauge transformations at $(A(t), \psi(t), \mathfrak{p})$ we may define the following operator

$$
\begin{gathered}
\mathcal{D}_{A, \psi, \mathfrak{p}}: \quad \mathcal{P}_{c} \oplus L_{k, \delta}^{2}\left(i \Omega^{1} \oplus W^{+}\right) \rightarrow L_{k-1, \delta}^{2}\left(i \Omega^{0} \oplus i \Omega^{2,+} \oplus W^{-}\right), \\
\mathcal{D}_{A, \psi, \mathfrak{p}}(\tilde{p}, a, \phi)=\left(G_{(A, \psi), \delta}^{*}, d^{+} a-(d t \wedge \sigma(\phi, \psi))^{+}+\mathfrak{p}^{+}, \not D_{A} \phi+\frac{1}{2} a . \psi+\mathfrak{p}_{\psi}\right),
\end{gathered}
$$

where $\left(\mathfrak{p}^{+}, \mathfrak{p}_{\psi}\right)$ is given by the linearization of the perturbation term at $(A(t), \psi(t), \mathfrak{p})$.

Let $\pi: Y \times \mathbb{R} \rightarrow Y$ be the projection map, then as bundles over $Y \times \mathbb{R}$,

$$
\begin{gathered}
\Lambda^{1}(Y \times \mathbb{R}, i \mathbb{R}) \cong \pi^{*} \Lambda^{1}(Y, i \mathbb{R}) \oplus \pi^{*}\left(\Lambda^{0}(Y, i \mathbb{R})\right. \\
\Lambda^{2,+}(Y \times \mathbb{R}, i \mathbb{R}) \cong \pi^{*} \Lambda^{1}(Y, i \mathbb{R})
\end{gathered}
$$


Also, using Clifford multiplication by $d t$, we identify $W^{+} \cong W^{-} \cong \pi^{*} W$. Under these identifications, fix $\mathfrak{p}$, then the operator $\mathcal{D}_{A, \psi, \mathfrak{p}}$, restricted to $L_{k, \delta}^{2}\left(i \Omega^{1} \oplus W^{+}\right)$, has the form

$$
\frac{\partial}{\partial t}+\left(\begin{array}{cc}
L_{(A, \psi)} & G_{(A, \psi)} \\
G_{(A, \psi)}^{*} & 0
\end{array}\right)+\delta+o(1)
$$

where $L_{(A, \psi)}, G_{(A, \psi)}$ and $G_{(A, \psi)}^{*}$ are given by (13). By the result of Lockhart-McOwen [18], as $\mathfrak{p}$ varies through $\mathcal{P}_{c}, \mathcal{D}_{A, \psi, \mathfrak{p}}$, restricted to $L_{k, \delta}^{2}\left(i \Omega^{1} \oplus W^{+}\right)$, forms a continuous family of Fredholm operators with index equal to $\mathfrak{i}\left(\gamma_{0}\right)-d_{\alpha}$.

Now we prove that $\mathcal{D}_{A(t), \psi(t), \mathfrak{p}}$ is surjective. Note that any solution $(A(t), \psi(t))$ which represents a point in $\mathcal{M}_{\mathfrak{p}}(\alpha, \beta)_{\gamma_{0}}$, is a solution to the perturbed gradient flow equation (27) with finite energy $\mathcal{C}_{\eta}\left(A_{\alpha}, \psi_{\alpha}\right)-\mathcal{C}_{\eta}\left(A_{\beta}, \psi_{\beta}\right)$. We can use Condition 3.12, to assert that there is a compact subset $[-T, T] \times Y$ in $\mathbb{R} \times Y$, such that outside this compact set, the perturbation function $\mathfrak{p} \in \mathcal{P}_{c}$ is zero.

Suppose that $(f, b, \phi) \in L_{-\delta}^{2}\left(i \Omega^{0} \oplus i \Omega^{2,+} \oplus W^{-}\right)$is $L^{2}$-orthogonal to the image of $\mathcal{D}_{A(t), \psi(t), \mathfrak{p}}$, then using Lemma 3.11 and a direct calculation shows that $(f, b, \phi)$ is zero on $[-T, T] \times Y$. As an element in the cokernel of $\mathcal{D}_{A(t), \psi(t), \mathfrak{p}},(f, b, \phi)$ satisfies the weak unique continuation principle [3] and hence $(f, b, \phi)$ is zero everywhere. This proves that $\mathcal{D}_{A(t), \psi(t), \mathfrak{p}}$ is surjective.

The Sard-Smale theorem implies that there exists a Baire set $\mathcal{P}_{0}$ of functions $\mathfrak{p} \in \mathcal{P}_{c}$, so that the moduli space $\mathcal{M}_{\mathfrak{p}}(\alpha, \beta)_{\gamma_{0}}$, for $\mathfrak{p} \in \mathcal{P}_{0}$, is a smooth manifold of dimension $\mathfrak{i}\left(\gamma_{0}\right)-d_{\alpha}$ if $\mathfrak{i}\left(\gamma_{0}\right)-d_{\alpha}>0$, and is empty otherwise.

The orientation of $\mathcal{M}_{\mathfrak{p}}(\alpha, \beta)_{\gamma_{0}}$ is determined by the determinant line bundle of $\mathcal{D}_{A, \psi, \mathfrak{p}}$ over $\mathcal{A}_{k, \delta}(\alpha, \beta)$ for a fixed perturbation $\mathfrak{p}$. This is established in Proposition 2.15 of 20 where it is shown that $\mathcal{M}_{\mathfrak{p}}(\alpha, \beta)_{\gamma_{0}}$ is orientable, the orientation is determined by choosing an orientation of

$$
\wedge^{t o p} H_{\delta}^{1}(Y \times \mathbb{R}, i \mathbb{R}) \otimes \wedge^{t o p} H_{\delta}^{2,+}(Y \times \mathbb{R}, i \mathbb{R})
$$

Proposition 3.16 has an immediate corollary.

Corollary 3.17. Let $(Y, g, \mathfrak{s})$ be a closed, oriented 3-manifold with a Riemannian metric $g$ and a $\operatorname{Spin}^{c}$ structure $\mathfrak{s}$. Let $\mathcal{M}(\alpha, \beta)$ be the moduli space of gradient flows of $\mathcal{C}_{\eta, \mathfrak{p}}$ which connect two different critical points $\alpha$ and $\beta$. Then, for a generic perturbation $\mathfrak{p}$, we have the following results.

1. When $Y$ is a homology 3-sphere, $\mathcal{M}(\alpha, \beta)$ is empty if $\mathfrak{i}(\alpha)-\mathfrak{i}(\beta)-d_{\alpha} \leq 0$, otherwise, $\mathcal{M}(\alpha, \beta)$ is an oriented, smooth manifold of dimension $\mathfrak{i}(\alpha)-\mathfrak{i}(\beta)-d_{\alpha}$. 
2. If $c_{1}(\mathfrak{s})$ is non-zero in $H^{2}(Y, \mathbb{R}), \mathcal{M}(\alpha, \beta)$ has infinitely many components. Each of them is an oriented, smooth manifold with dimension given by $\mathfrak{i}(\gamma)-d_{\alpha}>0$, where $\gamma$ is a chosen element in that component. The dimensions of two non-empty components differ by a multiple of $d(\mathfrak{s})$, where $d(\mathfrak{s})$ is the divisibility of $c_{1}(\mathfrak{s})$ in $H^{2}(Y, \mathbb{Z}) /$ Torsion.

Now we discuss the compactness of the trajectory moduli space. For the case of a 3-manifold $Y$ with $b_{1}(Y)>0$ and $c_{1}(\mathfrak{s}) \neq 0$ in $H^{2}(Y, \mathbb{R})$, we know that $\hat{\mathcal{M}}(\alpha, \beta)$, the quotient of $\mathcal{M}(\alpha, \beta)$ by $\mathbb{R}$, has infinitely many components. Assume $\mathfrak{i}(\alpha)-\mathfrak{i}(\beta)=k+1(\bmod d(\mathfrak{s}))$ with $k \geq 0$, then

$$
\hat{\mathcal{M}}(\alpha, \beta)=\cup_{n \in \mathbb{N}} \hat{\mathcal{M}}^{k+n d(\mathfrak{s})}(\alpha, \beta)
$$

where $\hat{\mathcal{M}}^{k+n d(\mathfrak{s})}(\alpha, \beta)$ is the union of components of dimension $k+n d(\mathfrak{s})$ in $\hat{\mathcal{M}}(\alpha, \beta)$. We now summarize the compactness results and refer to page 501-549 in [20] for the proofs.

Proposition 3.18. (Theorem 4.23 [20) For any 3-manifold $(Y, g, \mathfrak{s})$ with a Riemannian metric $g$ and $a \operatorname{Spin}^{c}$ structure $\mathfrak{s}$, assume $c_{1}(\mathfrak{s}) \neq 0$ if $b_{1}(Y)>0$, then $\hat{\mathcal{M}}^{k+n d(\mathfrak{s})}(\alpha, \beta)(\hat{\mathcal{M}}(\alpha, \beta)$ for $b_{1}(Y)=0$ ) can be compactified by adding lower dimensional boundary strata of broken trajectory moduli spaces. Namely, for $b_{1}(Y)=0$, the boundary strata are of the form

$$
\bigcup_{\alpha_{1}, \cdots \alpha_{j}} \hat{\mathcal{M}}\left(\alpha, \alpha_{1}\right) \times \hat{\mathcal{M}}\left(\alpha_{1}, \alpha_{2}\right) \times \cdots \times \hat{\mathcal{M}}\left(\alpha_{j}, \beta\right) .
$$

Here the union is over all possible sequences of critical points $\alpha, \alpha_{1}, \cdots \alpha_{j}, \beta$ with decreasing indices. For $b_{1}(Y)>0$ and $c_{1}(Y) \neq 0, \hat{\mathcal{M}}^{k+n d(\mathfrak{s})}(\alpha, \beta)$ can be compactified by adding boundary strata of the form

$$
\bigcup_{\alpha_{1}, \cdots \alpha_{j}} \hat{\mathcal{M}}^{k_{0}}\left(\alpha, \alpha_{1}\right) \times \hat{\mathcal{M}}^{k_{1}}\left(\alpha_{1}, \alpha_{2}\right) \times \cdots \times \hat{\mathcal{M}}^{k_{j}}\left(\alpha_{j}, \beta\right) .
$$

Here the union is over all possible sequences of critical points $\alpha, \alpha_{1}, \cdots \alpha_{j}, \beta$ with $k_{0}+k_{1}+\cdots+k_{j} \leq k+n d(\mathfrak{s})-j$ and $k_{i}=\mathfrak{i}\left(\alpha_{i}, \alpha_{i+1}\right)(\bmod d(\mathfrak{s}))$.

With these compactness results for the moduli space, we have the following corollary which will be crucial for the construction of Seiberg-Witten-Floer homology.

Corollary 3.19. 1. Let $(Y, \mathfrak{s})$ be a homology 3-sphere or $b_{1}(Y)>0$ with $c_{1}(\mathfrak{s}) \neq 0$, then the zero dimensional components $\hat{\mathcal{M}}^{0}(\alpha, \beta)$ for two critical points $\alpha$ and $\beta$ of relative index 1 are compact, and consist of finitely many oriented points.

2. Let $(Y, \mathfrak{s})$ be a homology 3-sphere or $b_{1}(Y)>0$ with $c_{1}(\mathfrak{s}) \neq 0$, let $\alpha, \gamma$ be two irreducible critical points in $\mathcal{M}_{Y}(\mathfrak{s}, \eta)$ with relative index $\mathfrak{i}(\alpha, \gamma)=2$, or $2 \bmod d(\mathfrak{s})$ for $b_{1}(Y)>0$. Then the boundary of $\hat{\mathcal{M}}^{1}(\alpha, \gamma)$ (an oriented, compact 1-manifold) consists of the union

$$
\bigcup_{\beta \in \mathcal{M}_{\mathfrak{s}, \mathfrak{p}}^{*}} \hat{\mathcal{M}}^{0}(\alpha, \beta) \times \hat{\mathcal{M}}^{0}(\beta, \gamma)
$$


where $\beta$ runs over those critical points $\beta$ with relative index $\mathfrak{i}(\alpha, \beta)=1$ or $1 \bmod d(\mathfrak{s})$ for $b_{1}(Y)>0$.

Remark 3.20. For a 3-manifold $(Y, \mathfrak{s})$ with $b_{1}(Y)>0$ and $c_{1}(\mathfrak{s})=0$, we need to perturb by a coclosed 2-form $\eta$ such that $[* \eta]$ representing a non-trivial cohomology class. Then $\mathcal{C}_{\eta}$ has finitely many critical points $\mathcal{M}_{Y}(\mathfrak{s}, \eta)$, which are all irreducible and have $\mathbb{Z}$-valued indices. Note that in this case $\mathcal{C}_{\eta}(g \cdot(A, \psi))-\mathcal{C}_{\eta}(A, \psi)=\langle[g] \cup[\eta],[Y]\rangle$, which is a multiple of a positive number $e_{\eta}$ $\left(e_{\eta}\right.$ depends on $\left.\eta\right)$. For two critical points $\alpha$ and $\beta$ with relative index $k+1>0$, and for generic $\mathfrak{p}, \hat{\mathcal{M}}(\alpha, \beta)$ is an oriented and smooth manifold of dimension $k, \hat{\mathcal{M}}(\alpha, \beta)=\bigcup_{n \in \mathbb{N}} \hat{\mathcal{M}}_{(n)}(\alpha, \beta)$ where $\hat{\mathcal{M}}_{(0)}(\alpha, \beta)$ is the union of components in $\hat{\mathcal{M}}(\alpha, \beta)$ with minimal energy $e_{0}(\alpha, \beta)$, and for $n>0, \hat{\mathcal{M}}_{(n)}(\alpha, \beta)$ is the the union of components in $\hat{\mathcal{M}}(\alpha, \beta)$ with energy $e_{0}(\alpha, \beta)+n e_{\eta}$. Moreover $\hat{\mathcal{M}}_{(n)}(\alpha, \beta)$ can be compactified by adding boundary strata of the form

$$
\bigcup_{\alpha_{1}, \cdots, \alpha_{l}} \hat{\mathcal{M}}_{\left(i_{0}\right)}\left(\alpha, \alpha_{1}\right) \times \hat{\mathcal{M}}_{\left(i_{1}\right)}\left(\alpha_{1}, \alpha_{2}\right) \times \cdots \times \hat{\mathcal{M}}_{\left(i_{l}\right)}\left(\alpha_{l}, \beta\right)
$$

Here the union is over all possible sequences of $\alpha_{0}=\alpha, \alpha_{1}, \cdots, \alpha_{l}, \alpha_{l+1}=\beta$ with decreasing indices and $\sum_{j=0}^{l} e_{0}\left(\alpha_{j}, \alpha_{j+1}\right)+\sum_{j=0}^{l} i_{j} e_{\eta} \leq e_{0}(\alpha, \beta)+n e_{\eta}$.

\subsection{Seiberg-Witten-Floer homology: definition and properties}

In this section, we will only construct the Seiberg-Witten-Floer homology for $(Y, \mathfrak{s})$ when $(Y, \mathfrak{s})$ has $b_{1}(Y)>0$ and $c_{1}(\mathfrak{s}) \neq 0$. When $Y$ is a homology sphere, there is an equivariant SeibergWitten-Floer homology, developed in 20, which is a topological invariant.

Let $(Y, g, \mathfrak{s})$ be an oriented, closed 3-manifold with a Riemannian metric $g$ and a $\operatorname{Spin}^{c}$ structure $\mathfrak{s}$. For a generic $\eta$, the critical points of $\mathcal{C}_{\mathfrak{p}}$ consist of finitely many, non-degenerate points, which we denote by $\mathcal{M}_{Y}(\mathfrak{s}, \eta)$, all irreducible.

The Floer complex $C_{*}^{S W}(Y, \mathfrak{s})$ is generated freely by the critical points in $\mathcal{M}_{Y}(\mathfrak{s}, \eta)$ with a relative $d(\mathfrak{s})$-grading

$$
C_{*}(Y, \mathfrak{s})=\oplus_{\alpha \in \mathcal{M}_{Y}(\mathfrak{s}, \eta)} \mathbb{Z} .<\alpha>.
$$

The boundary operator $\partial$ on $C_{*}(Y, \mathfrak{s})$ is defined by

$$
\partial(<\alpha>)=\sum_{\beta \in \mathcal{M}_{Y}(\mathfrak{s}, \eta)} n_{\alpha \beta}<\beta>
$$

where $n_{\alpha \beta}$ is given by counting the points in $\hat{\mathcal{M}}^{0}(\alpha, \beta)$ (an oriented, smooth, compact 0-manifold from Corollary 3.19) with sign.

Lemma 3.21. $\partial \circ \partial=0$. 
Proof. By definition,

$$
\begin{aligned}
\partial^{2}(<\alpha>) & =\sum_{\beta \in \mathcal{M}_{Y}(\mathfrak{s}, \eta)} n_{\alpha \beta} \partial(<\beta>) \\
& =\sum_{\beta \in \mathcal{M}_{Y}(\mathfrak{s}, \eta)} \sum_{\gamma \in \mathcal{M}_{Y}(\mathfrak{s}, \eta)} n_{\alpha \beta} n_{\beta \gamma}<\gamma>
\end{aligned}
$$

where $\beta$ runs over the critical points with relative index $\mathfrak{i}(\alpha, \beta)=1 \bmod (\partial(\mathfrak{s})), \gamma$ runs over the critical points with relative index $\mathfrak{i}(\alpha, \gamma)=2 \bmod (\partial(\mathfrak{s}))$. We want to show that

$$
\sum_{\{\beta \mid \mathfrak{i}(\alpha, \beta)=1 \bmod (d(\mathfrak{s}))\}} n_{\alpha \beta} n_{\beta \gamma}=0
$$

for any $\gamma \in \mathcal{M}_{Y}(\mathfrak{s}, \eta)$ with $\mathfrak{i}(\alpha, \gamma)=2 \bmod (d(\mathfrak{s}))$. We know that the number

$$
\sum_{\{\beta \mid \mathfrak{i}(\alpha, \beta)=1 \bmod (d(\mathfrak{s}))\}} n_{\alpha \beta} n_{\beta \gamma}
$$

is the number of oriented boundary points of the 1-manifold $\hat{\mathcal{M}}^{1}(\alpha, \gamma)$ by Corollary 3.19 , and hence is zero.

Now we can define the Seiberg-Witten-Floer homology.

Definition 3.22. For any closed oriented 3-manifold $Y$ with a Spin $^{c}$ structure, the SeibergWitten-Floer homology is defined to be

$$
H F_{*}^{S W}(Y, \mathfrak{s})=H_{*}\left(C_{*}^{S W}(Y, \mathfrak{s}), \partial_{*}\right),
$$

which is a $\mathbb{Z}_{d(\mathfrak{s})}$-graded and finitely generated Abelian group.

Remark 3.23. The Seiberg-Witten-Floer homology can be thought as a refinement of the Seiberg-Witten invariant for a 3-manifold $Y$ with a $\operatorname{Spin}^{c}$ structure $\mathfrak{s}$, in the sense that the Seiberg-Witten invariant for $(Y, \mathfrak{s})$ is the Euler characteristic of the Seiberg-Witten-Floer homology $H F_{*}^{S W}(Y, \mathfrak{s})$.

The definition of $H F_{*}^{S W}(Y, \mathfrak{s})$ involves both the metric and perturbations. The following proposition shows that for a 3-manifold $Y$ with $b_{1}(Y)>0$ and $c_{1}(\mathfrak{s}) \neq 0$, the Seiberg-Witten-Floer homology is a topological invariant, independent of the metric and generic perturbations $\eta$ and $\mathfrak{p}$. We then show that there is an the action of $\mathbb{A}(Y)=\operatorname{Sym}^{*}\left(H_{0}(Y, \mathbb{Z})\right) \otimes \Lambda^{*}\left(H_{1}(Y, \mathbb{Z}) /\right.$ torsion $)$ on $H F_{*}^{S W}(Y, \mathfrak{s})$.

Proposition 3.24. For any closed oriented 3-manifold $Y$ with a $\operatorname{Spin}^{c}$ structure $\mathfrak{s}$ such that $c_{1}(\mathfrak{s})$ is non-torsion, then $H F_{*}^{S W}(Y, \mathfrak{s})$ is a topological invariant and there is an action of $\mathbb{Z}[U]$ on $H F_{*}^{S W}(Y, \mathfrak{s})$ where the $U$-action decreases the relative grading by 2 . 
Proof. The proof of topological invariance can be found in $[20$. The $\mathbb{Z}[U]$-module structure can also be extracted from the isomorphism between $H F_{*}^{S W}(Y, \mathfrak{s})$ and the corresponding equivariant Seiberg-Witten-Floer homology $H F_{*, U(1)}^{S W}(Y, \mathfrak{s})$. Here we sketch a more direct proof of the existence of a $\mathbb{Z}[U]$-module structure on $H F_{*}^{S W}(Y, \mathfrak{s})$. In subsection 5.3 of [20], there is an associated integer $m_{\alpha \gamma}$ for each pair of critical points $\alpha$ and $\gamma$ in $\mathcal{M}_{Y}(\mathfrak{s}, \eta)$ of relative index 2 $\bmod (d(\mathfrak{s}))$, defined as follows.

Let $\mathcal{M}^{2}(\alpha, \beta)$ be the 2-dimensional components of the moduli space of parametrized flowlines between $\alpha$ and $\gamma$. There is a $U(1)$-principal bundle over $\mathcal{M}^{2}(\alpha, \beta)$, denoted by $\tilde{\mathcal{M}}^{2}(\alpha, \beta)$, which is the based moduli space corresponding to $\mathcal{M}^{2}(\alpha, \beta)$. That is, fix a base point $\left(t_{0}, y_{0}\right) \in \mathbb{R} \times Y$ and a complex line $L_{y_{0}}$ in the fiber $W_{y_{0}}$ of the spinor bundle $W$ over $Y$ such that $L_{y_{0}}$ doesn't contain the spinor part of any critical point in $\mathcal{M}_{Y}(\mathfrak{s}, \eta)$. Such $L_{y_{0}}$ exists due to the fact that $\mathcal{M}_{Y}(\mathfrak{s}, \eta)$ consists of finitely many irreducible critical points. Note also that the space of such $L_{y_{0}}$ is path-connected. Then there is a complex line bundle $\mathcal{L}_{\alpha \gamma}$ over $\mathcal{M}^{2}(\alpha, \beta)$

$$
\mathcal{L}_{\alpha \gamma}=\tilde{\mathcal{M}}^{2}(\alpha, \beta) \times_{U(1)}\left(W_{y_{0}} / L_{y_{0}}\right)
$$

with a canonical section

$$
s_{\alpha \gamma}([\mathbb{A}, \Psi])=\left([\mathbb{A}, \Psi], \Psi\left(t_{0}, y_{0}\right)\right) .
$$

This canonical section determines a trivialization of $\mathcal{L}_{\alpha \gamma}$ away from a compact set (see Lemma 5.7 in [20]) by the choice of $L_{y_{0}}$. Then the relative Euler number of $\left(\mathcal{L}_{\alpha \gamma}, s_{\alpha \gamma}\right)$ defines an integer $m_{\alpha \gamma}$ for each pair of $\alpha$ and $\gamma$ with relative index $2 \bmod (d(\mathfrak{s}))$. The system of integers $\left\{m_{\alpha \gamma}: \mathfrak{i}(\alpha, \gamma)=2 \bmod (d(\mathfrak{s}))\right\}$ satisfies the following properties (Cf. Lemma 5.7 and Remark 5.8 in [20]): $m_{\alpha \gamma}$ is independent of the choices of base points and $L_{y_{0}}$, and for $\alpha$ and $\delta$ two critical points with relative index $3 \bmod (d(\mathfrak{s}))$, then

$$
\sum_{\{\beta \mid \mathbf{i}(\alpha, \beta)=1 \bmod (d(\mathfrak{s}))\}} n_{\alpha \beta} m_{\beta \delta}-\sum_{\{\gamma \mid \mathbf{i}(\alpha \gamma)=2 \bmod (d(\mathfrak{s}))\}} m_{\alpha \gamma} n_{\gamma \delta}=0 .
$$

Now we can define a map

$$
\begin{aligned}
& U: \quad C_{*}^{S W}(Y, \mathfrak{s}) \quad \longrightarrow \quad C_{*-2}^{S W}(Y, \mathfrak{s}) \\
& <\alpha>\quad \mapsto \quad \sum_{\{\gamma \mid \mathbf{i}(\alpha, \gamma)=1 \bmod (d(\mathfrak{s}))\}} m_{\alpha \gamma}<\gamma>\text {. }
\end{aligned}
$$

Identity (39) implies that $U$ induces a map on the Seiberg-Witten-Floer homology which decreases the relative grading by 2 . This is the $\mathbb{Z}[U]$ module structure on $H F_{*}^{S W}(Y, \mathfrak{s})$.

Now we define the action of $\mathbb{A}(Y)=\operatorname{Sym}^{*}\left(H_{0}(Y, \mathbb{Z})\right) \otimes \Lambda^{*}\left(H_{1}(Y, \mathbb{Z}) /\right.$ Torsion $)$ on $H F_{*}^{S W}(Y, \mathfrak{s})$. Note that $\operatorname{Sym}^{*}\left(H_{0}(Y, \mathbb{Z})\right) \cong \mathbb{Z}[U]$, so we only need to define an action of $\Lambda^{*}\left(H_{1}(Y, \mathbb{Z}) /\right.$ Torsion $)$ on $H F_{*}^{S W}(Y, \mathfrak{s})$. 
Let $\pi$ be an embedded loop in $Y$ which represents a non-torsion element in $H_{1}(Y, \mathbb{Z})$. For any $\alpha$ and $\beta$ two critical points in $\mathcal{M}_{Y}(\mathfrak{s}, \eta)$ with relative index $1 \bmod (d(\mathfrak{s}))$, let $\mathcal{M}^{1}(\alpha, \beta)$ denote the 1-dimensional components of the moduli space of parametrized flowlines between $\alpha$ and $\beta$. Then there is a universal line bundle over $\pi \times \mathcal{M}^{1}(\alpha, \beta)$ constructed as follows: for any point $y \in \pi$, the based moduli space with base point $\left(t_{0}, y\right)$ defines a $U(1)$-bundle over $\mathcal{M}^{1}(\alpha, \beta)$. Varying $y$ in $\pi$ gives rise to a universal line $\widetilde{\mathcal{M}_{\pi}^{1}(\alpha, \beta)}$ over $\pi \times \mathcal{M}^{1}(\alpha, \beta)$.

As in the proof of Proposition 3.24, choose a fixed base point $y_{0}$ and a complex line $L_{y_{0}} \subset W_{y_{0}}$, then there is an associated complex line bundle

$$
\mathcal{L}_{\alpha \beta}^{\pi}=\widetilde{\mathcal{M}_{\pi}^{1}(\alpha, \beta)} \times_{U(1)}\left(W_{y_{0}} / L_{y_{0}}\right)
$$

over $\pi \times \mathcal{M}^{1}(\alpha, \beta)$, endowed with a canonical section $s_{\alpha \beta}^{\pi}$ defined in a fashion similar to that in the proof of Proposition 3.24. This canonical section $s_{\alpha \beta}^{\pi}$ also defines a trivialization away from a compact set in $\pi \times \mathcal{M}^{1}(\alpha, \beta)$. The corresponding relative Euler number is denoted by $\pi_{\alpha \beta}$ : it counts the zeros of a generic section which agrees with the trivialization determined by $s_{\alpha \beta}^{\pi}$. The standard cobordism argument implies that $\pi_{\alpha \beta}$ is independent of the choice of embedded loop $\pi$, hence we can denote this relative Euler number as $[\pi]_{\alpha \beta}$ for any non-torsion element $[\pi] \in H_{1}(Y, \mathbb{Z})$. This defines a map of degree -1 on the Seiberg-Witten-Floer chain complex:

$$
\begin{aligned}
& {[\pi] \quad C_{*}^{S W}(Y, \mathfrak{s}) \quad \longrightarrow \quad C_{*-1}^{S W}(Y, \mathfrak{s})} \\
& \langle\alpha\rangle \quad \mapsto \quad \sum_{\{\beta \mid \mathbf{i}(\alpha, \beta)=1 \bmod (d(\mathfrak{s}))\}}[\pi]_{\alpha \beta}<\beta>.
\end{aligned}
$$

Proposition 3.25. $[\pi]$ is a chain map and satisfies $[\pi] \circ[\pi]=0$, hence it defines an action of $\Lambda^{*}\left(H_{1}(Y, \mathbb{Z}) /\right.$ Torsion $)$ on the Seiberg-Witten-Floer homology $H F_{*}^{S W}(Y, \mathfrak{s})$.

Proof. First, we show that $[\pi]$ is a chain map. Let $\alpha$ and $\gamma$ be two critical points of relative index $2 \bmod (d(\mathfrak{s}))$. Let $\mathcal{M}^{2}(\alpha, \gamma)^{*}$ be the induced partial compactification of the 2-dimensional components of $\mathcal{M}(\alpha, \gamma)$ obtained by adding the broken flowlines. Note that, $\mathcal{M}^{2}(\alpha, \gamma)^{*}$ consists of finitely many cylinders (homeomorphic to $S^{1} \times \mathbb{R}$ ), and finitely many bands (homeomorphic to $[0,1] \times \mathbb{R})$. The boundary ends in the bands correspond to the unparametrised broken flowlines

$$
\bigcup_{\{\beta \mid \mathbf{i}(\alpha, \beta)=1 \bmod (d(\mathfrak{s}))\}} \hat{\mathcal{M}}^{0}(\alpha, \beta) \times \hat{\mathcal{M}}^{0}(\beta, \gamma),
$$

where $\hat{\mathcal{M}}^{0}(\alpha, \beta)$ and $\hat{\mathcal{M}}^{0}(\beta, \gamma)$ are the quotients of $\mathcal{M}^{1}(\alpha, \beta)$ and $\hat{\mathcal{M}}^{1}(\beta, \gamma)$ by the $\mathbb{R}$-action respectively. There is a complex line bundle $\mathcal{L}_{\alpha \gamma}^{\pi}$ over $\pi \times \mathcal{M}^{2}(\alpha, \gamma)^{*}$ with a canonical section $s_{\alpha \gamma}^{\pi}$, constructed in a fashion similar to that of $\left(\mathcal{L}_{\alpha \beta}^{\pi}, s_{\alpha \beta}^{\pi}\right)$ in the proof of Proposition 3.24. Away from a compact set in $\pi \times \mathcal{M}^{2}(\alpha, \gamma)^{*}, s_{\alpha \gamma}^{\pi}$ is nowhere vanishing, hence it determines a 
trivialization of $\mathcal{L}_{\alpha \gamma}^{\pi}$ away from a compact set. The zeros of a section which agrees with $s_{\alpha \gamma}^{\pi}$ away from a compact set form an oriented smooth 1-manifold. Such sections we refer to as generic sections of $\left(\mathcal{L}_{\alpha \gamma}^{\pi}, s_{\alpha \gamma}^{\pi}\right)$. Looking at the boundary of this zero set we see that it consists of

$$
\bigcup_{\{\beta \mid \mathfrak{i}(\alpha, \beta)=1 \bmod (d(\mathfrak{s}))\}}\left(\hat{s}_{\alpha \beta}^{-1}(0) \times \hat{\mathcal{M}}^{0}(\beta, \gamma) \cup \mathcal{M}^{0}(\alpha, \beta) \times \hat{s}_{\beta \gamma}^{-1}(0)\right) .
$$

Here $\hat{s}_{\alpha \beta}$ and $\hat{s}_{\beta \gamma}$ are generic sections of $\left(\mathcal{L}_{\alpha \beta}^{\pi}, s_{\alpha \beta}^{\pi}\right)$ and $\left(\mathcal{L}_{\beta \gamma}^{\pi}, s_{\beta \gamma}^{\pi}\right)$ respectively. The counting of these boundary points implies that

$$
\sum_{\{\beta \mid \mathbf{i}(\alpha, \beta)=1 \bmod (d(\mathfrak{s}))\}}[\pi]_{\alpha \beta} n_{\beta \gamma}-\sum_{\left\{\beta^{\prime} \mid \mathbf{i}\left(\alpha, \beta^{\prime}\right)=1 \bmod (d(\mathfrak{s}))\right\}} n_{\alpha \beta^{\prime}}[\pi]_{\beta^{\prime} \gamma}=0 .
$$

Hence $[\pi]$ is a chain map so we have defined an action of $H_{1}(Y, \mathbb{Z}) /$ Torsion on $H F_{*}^{S W}(Y, \mathfrak{s})$.

To get $[\pi] \circ[\pi]=0$ on the chain level, we need to give another way of calculating the relative Euler number of $\left(\mathcal{L}_{\alpha \beta}^{\pi}, s_{\alpha \beta}^{\pi}\right)$ in terms of the following holonomy map:

$$
h_{\alpha \beta}^{\pi}: \quad \mathcal{M}^{1}(\alpha, \beta) \longrightarrow U(1)
$$

where $h_{\alpha \beta}^{\pi}([\mathbb{A}, \Psi])$ is given by the holonomy of $\mathbb{A}$ along the loop $\left\{t_{0}\right\} \times \pi$ for any $[\mathbb{A}, \Psi] \in \mathcal{M}^{1}(\alpha, \beta)$. Let $\omega$ be the standard closed 1-form on $U(1)$ with integral one over $U(1)$. Then pulling back:

$$
[\pi]_{\alpha \beta}=\int_{\mathcal{M}^{1}(\alpha, \beta)}\left(h_{\alpha \beta}^{\pi}\right)^{*} \omega
$$

which is the winding number of the holonomy map $h_{\alpha \beta}^{\pi}$, that is, the counting of a generic fiber of $h_{\alpha \beta}^{\pi}$.

Now for two critical points $\alpha$ and $\gamma$ with relative index $2 \bmod (d(\mathfrak{s}))$, the corresponding holonomy map can be extended over the partial compactification of $\mathcal{M}^{2}(\alpha, \gamma)^{*}$. We denote this holonomy map by $h_{\alpha \gamma}^{\pi}$. Then the fiber of $h_{\alpha \gamma}^{\pi}$ at a generic point $p \in U(1)$ is an oriented smooth 1-manifold whose boundary consists of

$$
\bigcup_{\{\beta \mid \mathfrak{i}(\alpha, \beta)=1 \bmod (d(\mathfrak{s}))\}}\left(h_{\alpha \beta}^{\pi}\right)^{-1}(p) \times\left(h_{\beta \gamma}^{\pi}\right)^{-1}(p) .
$$

Counting these boundary points with sign, we obtain

$$
\sum_{\{\beta \mid \mathbf{i}(\alpha, \beta)=1 \bmod (d(\mathfrak{s}))\}}[\pi]_{\alpha \beta}[\pi]_{\beta \gamma}=0 .
$$

This shows that $[\pi] \circ[\pi]=0$ on the chain level. Hence there is an action of $\Lambda^{*}\left(H_{1}(Y, \mathbb{Z}) /\right.$ Torsion $)$ on the Seiberg-Witten-Floer homology $H F_{*}^{S W}(Y, \mathfrak{s})$. 
Let $(-Y,-\mathfrak{s})$ be the 3 -manifold $Y$ with the reversed orientation. There is a one-to-one correspondence between the 3-dimensional monopoles on $(Y, \mathfrak{s})$ and $(-Y,-\mathfrak{s})$

$$
\mathcal{M}_{Y}(\mathfrak{s}, \eta) \cong \mathcal{M}_{-Y}(-\mathfrak{s},-\eta)
$$

which sends $\alpha \mapsto \hat{\alpha}$, such that

$$
\mathfrak{i}_{Y}(\alpha, \beta)=\mathfrak{i}_{-Y}(\hat{\beta}, \hat{\alpha})
$$

There is also an orientation preserving diffeomorphism between the moduli space of gradient flow lines for the Chern-Simons-Dirac functionals on $(Y, \mathfrak{s})$ and $(-Y,-\mathfrak{s})$ :

$$
\mathcal{M}_{\mathbb{R} \times Y}(\alpha, \beta) \cong \mathcal{M}_{\mathbb{R} \times-Y}(\hat{\beta}, \hat{\alpha})
$$

Hence from the construction of the Seiberg-Witten-Floer homology, we see that $C_{-*}(-Y,-\mathfrak{s})$ can be identified to the dual complex of $C_{*}(Y, \mathfrak{s})$. This gives rise to a natural pairing

$$
\langle,\rangle: \quad H F_{*}^{S W}(Y, \mathfrak{s}) \times H F_{-*}^{S W}(-Y,-\mathfrak{s}) \longrightarrow \mathbb{Z},
$$

which satisfies the following properties whose proofs are immediate from the definition:

1. For two relative degree 2 cycles $\Xi_{1}$ and $\Xi_{2}$ in $H F_{*}^{S W}(Y, \mathfrak{s})$ and $H F_{-*}^{S W}(-Y,-\mathfrak{s})$ respectively $<U\left(\Xi_{1}\right), \Xi_{2}>=<\Xi_{1}, U\left(\Xi_{2}\right)>$.

2. For two relative degree 1 cycles $\Xi_{1}$ and $\Xi_{2}$ in $H F_{*}^{S W}(Y, \mathfrak{s})$ and $H F_{-*}^{S W}(-Y,-\mathfrak{s})$ respectively, and any $[\pi] \in H_{1}(Y, \mathbb{Z}) /$ Torsion $\cong H_{1}(-Y, \mathbb{Z}) /$ Torsion $,<[\pi]\left(\Xi_{1}\right), \Xi_{2}>=<\Xi_{1},[\pi]\left(\Xi_{2}\right)>$.

Thus, $<z . \Xi_{1}, \Xi_{2}>=<\Xi_{1}, z . \Xi_{2}>$ for any $z \in \mathbb{A}(Y) \cong \mathbb{A}(-Y)$, for any cycles $\Xi_{1} \in H F_{*}^{S W}(Y, \mathfrak{s})$ and $\Xi_{2} \in H F_{-*}^{S W}(-Y,-\mathfrak{s})$ respectively.

\subsection{Variants of the Seiberg-Witten-Floer homology}

The Seiberg-Witten-Floer homology $H F_{*}^{S W}(Y, \mathfrak{s})$, as defined in the previous subsection, is a finitely generated $\mathbb{Z}_{d(\mathfrak{s})}$-graded Abelian group. There are various ways of defining $\mathbb{Z}$-graded Seiberg-Witten-Floer homology groups for any closed oriented 3-manifold $Y$ with a non-torsion $\operatorname{Spin}^{c}$ structure $\mathfrak{s}$. These were briefly discussed at the end of section 4.1 of [20]. In this subsection, we propose a way of defining $\mathbb{Z}$-graded Seiberg-Witten-Floer homology, which will be convenient for our later description of the relative Seiberg-Witten invariant for a 4-manifold with cylindrical end modelled on $([-2, \infty) \times Y, \mathfrak{s})$.

Notice that the first Chern class of the non-torsion $\operatorname{Spin}^{c}$ structure $\mathfrak{s}$ defines a homomorphism: $c_{1}(\mathfrak{s}): \quad H^{1}(Y, \mathbb{Z}) \longrightarrow \mathbb{Z}$ by $c_{1}(\mathfrak{s})([u])=<[u] \cup c_{1}(\mathfrak{s}),[Y]>$ for any element $[u] \in H^{1}(Y, \mathbb{Z})$.

For any subgroup $K \subseteq \operatorname{Ker}\left(c_{1}(\mathfrak{s})\right)$, there is a subgroup $\mathcal{G}_{Y}^{K}$ of the full gauge transformation group $\mathcal{G}_{Y}$, whose elements lie in the connected components determined by $K$ (note that the 
group of connected components of $\mathcal{G}_{Y}$ is $H^{1}(Y, \mathbb{Z})$ ). Consider the Seiberg-Witten-Floer homology theory for the Chern-Simons-Dirac functional on the configuration space $\mathcal{A}_{Y}$ modulo the gauge group $\mathcal{G}_{Y}^{K}$. The critical point set, denoted by $\mathcal{M}_{Y, K}(\mathfrak{s}, \eta)$, is a covering space

$$
\pi_{K}: \quad \mathcal{M}_{Y, K}(\mathfrak{s}, \eta) \longrightarrow \mathcal{M}_{Y}(\mathfrak{s}, \eta)
$$

whose fiber is an $H^{1}(Y, \mathbb{Z}) / K$-homogeneous space, hence, there is a natural action of $H^{1}(Y, \mathbb{Z}) / K$ on $\mathcal{M}_{Y, K}(\mathfrak{s}, \eta)$.

The generators of this variant of the Seiberg-Witten-Floer chain complex are elements in $\mathcal{M}_{Y, K}(\mathfrak{s}, \eta)$, with relative $\mathbb{Z}$-graded indices. Denote this chain complex by

$$
C_{*,[K]}^{S W}(Y, \mathfrak{s})=\sum_{\Gamma \in \mathcal{M}_{Y, K}(\mathfrak{s}, \eta)} \mathbb{Z}<\Gamma>
$$

The boundary operator $\partial^{K}$ is given by counting the gradient flowlines of the perturted CSD functional on $\mathcal{A}_{Y} / \mathcal{G}_{Y}^{K}$ connecting two critical points with relative index 1. Fix an element $\Gamma_{\alpha} \in \pi_{K}^{-1}(\alpha)$, for any critical point $\beta \in \mathcal{M}_{Y}(\mathfrak{s}, \eta)$, there is an orientation presevering diffeomorphism

$$
\mathcal{M}(\alpha, \beta) \cong \bigcup_{\Gamma_{\beta} \in \pi_{K}^{-1}(\beta)} \mathcal{M}\left(\Gamma_{\alpha}, \Gamma_{\beta}\right)
$$

where $\mathcal{M}\left(\Gamma_{\alpha}, \Gamma_{\beta}\right)$ is the moduli space of flowlines on $\mathcal{A}_{Y} / \mathcal{G}_{Y}^{K}$ connecting $\Gamma_{\alpha}$ and $\Gamma_{\beta}$. Hence, it is easy to see that there is a well-defined topological invariant, given by the homology of this new version of the Seiberg-Witten-Floer chain complex, though not finitely generated, still admitting an action of $\mathbb{A}(Y)$.

Definition 3.26. For any subgroup $K \subseteq \operatorname{Ker}\left(c_{1}(\mathfrak{s})\right)$, there is a variant of the Seiberg-WittenFloer chain complex, whose generators are elements from the covering space $\mathcal{M}_{Y, K}(\mathfrak{s}, \eta)$ of $\mathcal{M}_{Y}(\mathfrak{s}, \eta)$, and the boundary operator is given by counting the gradient flowlines of the perturbed CSD functional on $\mathcal{A}_{Y} / \mathcal{G}_{Y}^{K}$ connecting two critical points with relative index 1 . We denote the Seiberg-Witten-Floer homology in this setting by $\operatorname{HF}_{*,[K]}^{S W}(Y, \mathfrak{s})$, the actions of elements in $H_{0}(Y, \mathbb{Z})$ and $H_{1}(Y, \mathbb{Z}) /$ Torsion decreasing degree in $H F_{*,[K]}^{S W}(Y, \mathfrak{s})$ by 2 and 1 respectively.

From the definition, it is easy to get the following periodicity property for $H F_{*,\left[K \operatorname{Ker}\left(c_{1}(\mathfrak{s})\right)\right]}^{S W}(Y, \mathfrak{s})$ :

$$
H F_{m,\left[\operatorname{Ker}\left(c_{1}(\mathfrak{s})\right)\right]}^{S W}(Y, \mathfrak{s}) \cong H F_{m}^{S W} \bmod (d(\mathfrak{s}))(Y, \mathfrak{s}),
$$

for any $m \in \mathbb{Z}$.

Remark 3.27. From the natural action of $H_{1}(Y, \mathbb{Z}) / K$ on the generators of the chain complex $C_{*,[K]}^{S W}(Y, \mathfrak{s}), H F_{*,[K]}^{S W}(Y, \mathfrak{s})$ can be thought as an $H_{1}(Y, \mathbb{Z}) / K$-equivariant Seiberg-Witten-Floer 
homology of $(Y, \mathfrak{s})$. For any $[u] \in H_{1}(Y, \mathbb{Z}) / K$, the action of $[u]$ on $H F_{*,[K]}^{S W}(Y, \mathfrak{s})$ is the following $\mathbb{A}(Y)$-equivariant homomorphism:

$$
[u]: \quad H F_{*,[K]}^{S W}(Y, \mathfrak{s}) \longrightarrow H F_{*-n,[K]}^{S W}(Y, \mathfrak{s})
$$

with $n=<[u] \wedge c_{1}(\mathfrak{s}),[Y]>$.

For $(-Y,-\mathfrak{s})$, where $-Y$ is $Y$ with the reversed orientation and $-\mathfrak{s}$ is the induced $\operatorname{Spin}^{c}$ structure, the corresponding Seiberg-Witten-Floer complex $C_{*,[K]}^{S W}(-Y,-\mathfrak{s})$ is the dual complex of $C_{*,[K]}^{S W}(Y, \mathfrak{s})$. This gives a natural pairing

$$
\langle,\rangle: \quad H F_{*,[K]}^{S W}(Y, \mathfrak{s}) \times H F_{-*,[K]}^{S W}(-Y,-\mathfrak{s}) \longrightarrow \mathbb{Z}
$$

satisfying $<z . \Xi_{1}, \Xi_{2}>=<\Xi_{1}, z \cdot \Xi_{2}>$ for any $z \in \mathbb{A}(Y) \cong \mathbb{A}(-Y)$ and any cycles $\Xi_{1} \in H F_{*,[K]}^{S W}(Y, \mathfrak{s})$ and $\Xi_{2} \in H F_{-*,[K]}^{S W}(-Y,-\mathfrak{s})$ respectively.

For any subgroup $K$ in $\operatorname{Ker}\left(c_{1}(\mathfrak{s})\right)$, there is a chain map $C_{*,[K]}^{S W}(Y, \mathfrak{s}) \rightarrow C_{*}^{S W}(Y, \mathfrak{s})$ induced from the map $\pi_{K}$, which descends to an $\mathbb{A}(Y)$-equivariant homomorphism

$$
\pi_{K}: \quad H F_{*,[K]}^{S W}(Y, \mathfrak{s}) \longrightarrow H F_{*}^{S W}(Y, \mathfrak{s}) .
$$

Let $K_{1} \subset K_{2}$ be two subgroups in $\operatorname{Ker}\left(c_{1}(\mathfrak{s})\right)$. There is a covering map

$$
\pi: \quad \mathcal{M}_{Y, K_{1}}(\mathfrak{s}, \eta) \longrightarrow \mathcal{M}_{Y, K_{2}}(\mathfrak{s}, \eta)
$$

which induces a map:

$$
\pi_{*}: \quad C_{*,\left[K_{1}\right]}^{S W}(Y, \mathfrak{s}) \longrightarrow C_{*,\left[K_{2}\right]}^{S W}(Y, \mathfrak{s})
$$

assigning the generator $\left\langle\Gamma>\right.$ of $C_{*,\left[K_{1}\right]}(Y, \mathfrak{s})$ to $<\pi(\Gamma)>$ in $C_{*,\left[K_{2}\right]}(Y, \mathfrak{s})$. For any $\alpha, \beta$ in $\mathcal{M}_{Y, K_{2}}(\mathfrak{s}, \eta)$ of relative index 1 , and for any fixed $\Gamma_{\alpha}$ in $\pi^{-1}(\alpha)$, there is an orientation preserving diffeomorphism:

$$
\mathcal{M}_{\mathbb{R} \times Y}(\alpha, \beta) \cong \bigcup_{\Gamma_{\beta} \in \pi^{-1}(\beta)} \mathcal{M}_{\mathbb{R} \times Y}\left(\Gamma_{\alpha}, \Gamma_{\beta}\right) .
$$

which implies that $\pi_{*}$ is a chain map and induces an $\mathbb{A}(Y)$-equivariant homomorphism

$$
\pi: \quad H F_{*,\left[K_{1}\right]}^{S W}(Y, \mathfrak{s}) \longrightarrow H F_{*,\left[K_{2}\right]}^{S W}(Y, \mathfrak{s}) .
$$

For any two subgroups $K_{1}$ and $K_{2}$ in $\operatorname{Ker}\left(c_{1}(\mathfrak{s})\right)$, we have the following commutative diagram which relates various covering spaces of $\mathcal{M}_{Y}(\mathfrak{s}, \eta)$ :

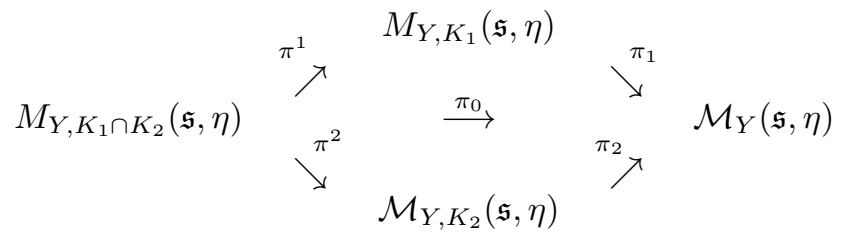


where the fibers of $\pi_{0}, \pi^{1}, \pi_{1}, \pi^{2}$ and $\pi_{2}$ are the homogeneous spaces of $H^{1}(Y, \mathbb{Z}) /\left(K_{1} \cap K_{2}\right)$, $K_{1} /\left(K_{1} \cap K_{2}\right), H^{1}(Y, \mathbb{Z}) / K_{1}, K_{2} /\left(K_{1} \cap K_{2}\right)$ and $H^{1}(Y, \mathbb{Z}) / K_{2}$ respectively.

Fix an element in $\mathcal{M}_{Y, K_{1} \cap K_{2}}(\mathfrak{s}, \eta)$, then there is a well-defined $\mathbb{Z}$-graded index on $\mathcal{M}_{Y, K_{1} \cap K_{2}}(\mathfrak{s}, \eta)$, as $K_{1}$ and $K_{2}$ are two subgroups of $\operatorname{Ker}\left(c_{1}(\mathfrak{s})\right)$. Hence there are the induced $\mathbb{Z}$-graded indices on $\mathcal{M}_{Y, K_{1}}(\mathfrak{s}, \eta)$ and $\mathcal{M}_{Y, K_{2}}(\mathfrak{s}, \eta)$. The $\mathcal{A}(Y)$-equivariant homomorphisms as provided in (45) and (44) induce the following commutative diagram of $\mathbb{A}(Y)$-equivariant homomorphisms.

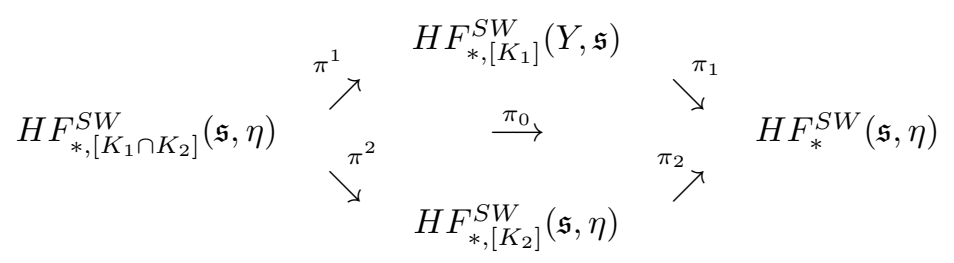

\section{Gluing formulae for 4-dimensional Seiberg-Witten in- variants}

In this Section, we will study the Seiberg-Witten invariant of a closed smooth 4-manifold $X$ with $b_{2}^{+}(X)>1$ and Spin $^{c}$ structure $\mathfrak{s}$ by stretching its metric along a smooth embedded separating 3-manifold $Y$ with the condition that $\left.\mathfrak{s}\right|_{Y}$ is non-torsion. Consider a 1-parameter family of metrics $\left\{g_{R}\right\}_{R>0}$ on $X$ such that for each $\left(X, g_{R}\right)$, there is an isometrical embedding

$$
\left([-R-2, R+2] \times Y, d t^{2}+g_{Y}\right) \longrightarrow\left(X, g_{R}\right) .
$$

Write for brevity, $X(R)=\left(X, g_{R}\right)$.

As $Y$ separates $X=X(0)$, we write $X(0)=X_{+} \cup X_{-}$with $Y=\{0\} \times Y=X_{+} \cap X_{-}$. There is a canonical orientation preserving isometry

$$
\Theta_{R}: \quad X(R)-\{0\} \times Y \longrightarrow X_{+}(R) \cup X_{-}(R),
$$

where $X_{+}(R)=X_{+} \cup[0, R] \times Y, X_{-}(R)=X_{-} \cup[-R, 0] \times Y$. As $R \rightarrow \infty, X_{ \pm}(R)$ become 4 -manifolds with cylindrical ends modelled on $[-2, \infty) \times Y$ and $(-\infty, 2] \times Y$. We denote these 4-manifolds by $X_{ \pm}(\infty)$.

Now, it is generally believed that, in some favorable cases, the moduli space of the SeibergWitten monopoles on $X(R)$, for a sufficiently large $R$, can be obtained by gluing together the moduli spaces for $X_{ \pm}(\infty)$ over the ends. In this section, we will establish this gluing theorem and give an expression for the Seiberg-Witten invariant of $X$ in terms of certain relative SeibergWitten invariants for $X_{ \pm}(\infty)$.

First we need to study the moduli space of Seiberg-Witten monopoles on $X_{ \pm}(\infty)$ with $\operatorname{Spin}^{c}$ structures $\mathfrak{s}_{ \pm}$which are the pull-back $\operatorname{Spin}^{c}$ structures when restricted to the ends. We 
assume that $\left.\mathfrak{s}_{ \pm}\right|_{Y}= \pm \mathfrak{t}$ is a non-torsion $\operatorname{Spin}^{c}$ structure. Then we will define relative SeibergWitten invariants for $\left(X_{ \pm}(\infty), \mathfrak{s}_{ \pm}\right)$which take values in certain variants of the Seiberg-WittenFloer homology groups $H F_{*}^{S W}(Y, \mathfrak{t})$ and $H F_{*}^{S W}(-Y,-\mathfrak{t})$ respectively. As an application of the Seiberg-Witten-Floer homology, we will prove the gluing formula for the Seiberg-Witten invariants on $X$ as claimed in Theorem 1.2 .

\subsection{Moduli space for 4-manifolds with cylindrical ends}

Let $X_{+}(\infty)$ be a Riemannian 4-manifold with a cylindrical end modelled on $[-2, \infty) \times Y$ and endowed with a Spin ${ }^{c}$ structure $\mathfrak{s}_{+}$such that $\left.\mathfrak{s}_{+}\right|_{[-2, \infty) \times Y}$ is the pull-back Spin ${ }^{c}$ structure of a non-torsion $\operatorname{Spin}^{c}$ structure $\mathfrak{t}$ on $Y$. Associated to $\mathfrak{s}_{+}$, there is a pair of rank 2 Hermitian bundles $W^{ \pm}$on $X_{+}(\infty)$, a complex line bundle $\operatorname{det}(\mathfrak{s})=\operatorname{det}\left(W^{ \pm}\right)$, and a Clifford multiplication endomorphism

$$
T^{*} X_{+}(\infty) \otimes W^{ \pm} \longrightarrow W^{\mp}
$$

Using the Clifford multiplication of $d t$ over $[-2, \infty) \times Y, W^{ \pm}$can be identified with a rank 2 Hermitian bundle $W$ on $Y$, and there is an induced Clifford multiplication homomorphism

$$
\rho: \quad T^{*} Y \times W \longrightarrow W
$$

Then $\mathfrak{t}=(W, \rho)$ is the $\operatorname{Spin}^{c}$ structure on $Y$ as in the Definition 2.1.

The perturbed Seiberg-Witten equations on $\left(X_{+}(\infty), \mathfrak{s}_{+}\right)$are the following equations, for a pair consisting of an $L_{2, l o c}^{2}$-connection $\mathbb{A}$ on $\operatorname{det}(\mathfrak{s})$ and an $L_{2, l o c}^{2}$-section $\Psi$ in $W^{+}$, given by

$$
\left\{\begin{array}{l}
F_{\mathbb{A}}^{+}=q(\Psi, \Psi)+\omega+\chi\left(\eta^{+}+\mathfrak{p}_{\mathbb{A}}^{+}\right) \\
\not D_{\mathbb{A}} \Psi=\chi \mathfrak{p}_{\Psi} .
\end{array}\right.
$$

Here $\omega$ is an imaginary valued self-dual 2 -form supported in a non-empty open set in $X_{+}(-2)=X_{+}(0)-[-2,0] \times Y, \chi$ is a cut-off function on $X_{+}(\infty)$ which is 1 on $[0, \infty) \times Y$ and 0 on $X_{+}(-2)$, and $\left(\eta^{+}+\mathfrak{p}_{\mathbb{A}}^{+}, \mathfrak{p}_{\Psi}\right)$ is the perturbation of the Seiberg-Witten equations on $[-2, \infty) \times Y$ as written in (30) (where $\eta$ and $\mathfrak{p}$ satisfy Remark (3.4) and Condition (3.12) in their Baire sets). The perturbed Seiberg-Witten equations (49) are invariant under the action of the gauge group, which is the group of $L_{3, l o c}^{2}$-maps from $X_{+}(\infty)$ to $U(1)$.

Let $(\mathbb{A}, \Psi)$ be a solution to the perturbed Seiberg-Witten equations (49) on $\left(X_{+}(\infty), \mathfrak{s}_{+}\right)$, in temporal gauge when restricted to the end $[-2, \infty) \times Y$. The corresponding path, which is a gradient flowline of $\mathcal{C}_{\eta, \mathfrak{p}}$, is denoted by $\gamma(t)=(A(t), \psi(t)):[-2, \infty) \rightarrow \mathcal{A}_{Y}$. We say that $(\mathbb{A}, \Psi)$ has finite energy if

$$
\lim _{t \rightarrow \infty}\left(\mathcal{C}_{\eta, \mathfrak{p}}(\gamma(-2))-\mathcal{C}_{\eta, \mathfrak{p}}(\gamma(t))\right)
$$


exists and is finite. The finite energy condition is equivalent to the following condition (see Lemma 3.13):

$$
\int_{-2}^{\infty}\left(\left\|\frac{\partial A}{\partial t}\right\|_{L^{2}(Y)}^{2}+\left\|\frac{\partial \psi}{\partial t}\right\|_{L^{2}(Y)}^{2}\right) d t<\infty
$$

Any solution to the perturbed Seiberg-Witten equations (49) on $\left(X_{+}(\infty), \mathfrak{s}_{+}\right)$is smooth. We put a topology on the set of solutions by its embedding to

$$
L_{2, l o c}^{2}\left(\Omega^{1}\left(X_{+}(\infty), i \mathbb{R}\right) \oplus \Gamma\left(W^{+}\right)\right) \oplus \mathbb{R}
$$

where the last component is given by $\int_{-2}^{\infty}\left(\left\|\frac{\partial A}{\partial t}\right\|_{L^{2}(Y)}^{2}+\left\|\frac{\partial \psi}{\partial t}\right\|_{L^{2}(Y)}^{2}\right) d t$ for the associated path $(A(t), \psi(t))$ determined by restricting a solution $(\mathbb{A}, \Psi)$ to $[-2, \infty) \times Y$ (which is in temporal gauge). Under this topology, the action of the $L_{3, l o c}^{2}$ gauge group is smooth on the set of finite energy solutions.

The moduli space of 4-dimensional monopoles on $\left(X_{+}(\infty), \mathfrak{s}_{+}\right)$is defined to be the quotient of the set of finite energy solutions to (49) by the action of the $L_{3, \text { loc }}^{2}$ gauge group with the quotient topology. Denote by $\mathcal{M}_{X_{+}}\left(\mathfrak{s}_{+}\right)$the moduli space of monopoles on $\left(X_{+}(\infty), \mathfrak{s}_{+}\right)$.

By Lemma 3.13 and Lemma 3.14, we know that any finite energy solutions to (49) decay exponentially to a 3 -dimensional Seiberg-Witten monopole representing a critical point in $\mathcal{M}_{Y}(\mathfrak{s}, \eta)$. The proof of the following proposition is straightforward.

Proposition 4.1. Let $\left(X_{+}(\infty), \mathfrak{s}^{+}\right)$be a complete Riemannian 4-manifold with a cylindrical end $[-2, \infty) \times Y$ and $a \operatorname{Spin}^{c}$ structure $\mathfrak{s}$ such that $\left.\mathfrak{s}\right|_{[-2, \infty) \times Y}$ is the pull-back $\operatorname{Spin}^{c}$ structure of a non-torsion $\operatorname{Spin}^{c}$ structure $\mathfrak{t}$ on $Y$. Let $(\mathbb{A}, \Psi)$ be a finite energy solution to the perturbed Seiberg-Witten equations (49)) on $\left(X_{+}(\infty), \mathfrak{s}_{+}\right)$, in temporal gauge when restricted to the end $[-2, \infty) \times Y$ and denoted by $(A(t), \psi(t))$. Then there is a constant $\delta>0$, and a constant $C>0$, such that there is a 3-dimensional monopole $\left(A_{\alpha}, \psi_{\alpha}\right)$ representing a critical point $\alpha \in \mathcal{M}_{Y}(\mathfrak{t}, \eta)$ for which

$$
\sum_{0 \leq k \leq 2}\left(\left|\nabla^{k}\left(A(t)-A_{\alpha}\right)\right|+\left|\left(\nabla_{A_{\alpha}}\right)^{k}\left(\psi(t)-\psi_{\alpha}\right)\right|\right) \leq C e^{-\delta t}
$$

for any point $(t, y) \in[0, \infty) \times Y$.

This proposition ensures that there is a boundary asymptotic limit map

$$
\partial_{\infty}: \quad \mathcal{M}_{X_{+}}\left(\mathfrak{s}_{+}\right) \longrightarrow \mathcal{M}_{Y, X_{+}}(\mathfrak{t}, \eta)
$$

where $\mathcal{M}_{Y, X_{+}}(\mathfrak{t}, \eta)$ is the quotient of solutions to the perturbed Seiberg-Witten equations (9) on $(Y, \mathfrak{t}, \eta)$ by the action of those gauge transformations on $Y$ which can be extended to $X_{+}(0)$. Note that $\mathcal{M}_{Y, X_{+}}(\mathfrak{t}, \eta)$ is a covering space of $\mathcal{M}_{Y}(\mathfrak{t}, \eta)$, whose fiber is an $H^{1}(Y, \mathbb{Z}) / \operatorname{Im}\left(i_{+}^{*}\right)$ homogeneous space, where $\operatorname{Im}\left(i_{+}^{*}\right)$ is the range of $i_{+}^{*}: H^{1}\left(X_{+}, \mathbb{Z}\right) \rightarrow H^{1}(Y, \mathbb{Z})$. Notice that 
$\operatorname{Im}\left(i_{+}^{*}\right)$ is a subgroup of $\operatorname{Ker}\left(c_{1}(\mathfrak{t})\right)$ where $c_{1}(\mathfrak{t})$ is the map from $H^{1}(Y, \mathbb{Z}) \rightarrow \mathbb{Z}$ defined in Subsection 3.5.

Over the end $[-2, \infty) \times Y$, if $\left(X_{+}, \mathfrak{s}_{+}\right)$is modelled on $(Y, \mathfrak{t}=(W, \rho))$ up to an isomorphism $u \in C^{\infty}(Y, U(1))$, the equivalent classes of such modelling is given by $[u] \in H^{1}(Y, \mathbb{Z}) / \operatorname{Im}\left(i_{+}^{*}\right)$ determined by the connected component of $C^{\infty}(Y, U(1))$ which $u$ belongs to. Then the corresponding asymptotic value map is given by $[u] \circ \partial_{\infty}$ with $[u] \in H^{1}(Y, \mathbb{Z}) / \operatorname{Im}\left(i_{+}^{*}\right)$ acting on $\mathcal{M}_{Y, X_{+}}(\mathfrak{t}, \eta)$.

Denote by $\pi_{+}$the covering map $\mathcal{M}_{Y, X_{+}}(\mathfrak{t}, \eta) \rightarrow \mathcal{M}_{Y}(\mathfrak{t}, \eta)$. Let $\mathcal{M}_{X_{+}}\left(\mathfrak{s}_{+}, \alpha\right)$ be the subspace of $\mathcal{M}_{X_{+}}\left(\mathfrak{s}_{+}\right)$whose elements decay in the $L_{2, \delta}^{2}$-topology to a 3 -dimensional monopole representing $\alpha$ in $\mathcal{M}_{Y}(\mathfrak{t}, \eta)$. Then we know that

$$
\mathcal{M}_{X_{+}}\left(\mathfrak{s}_{+}, \alpha\right)=\bigcup_{\Gamma_{\alpha} \in \pi_{+}^{-1}(\alpha)} \mathcal{M}_{X_{+}}\left(\mathfrak{s}_{+}, \Gamma_{\alpha}\right)
$$

where $\mathcal{M}_{X_{+}}\left(\mathfrak{s}_{+}, \Gamma_{\alpha}\right)=\partial_{\infty}^{-1}\left(\Gamma_{\alpha}\right)$ is the fiber of the boundary limit map (51) over $\Gamma_{\alpha}$. Then Proposition 4.1 tells us that $\mathcal{M}_{X_{+}}\left(\mathfrak{s}_{+}, \Gamma_{\alpha}\right)$ is the moduli space of solutions to the perturbed Seiberg-Witten equations (49) with $(\mathbb{A}, \Psi)$ belonging to

$$
\left(\mathbb{A}_{\alpha}, \Psi_{\alpha}\right)+L_{2, \delta}^{2}\left(\Omega^{1}\left(X_{+}(\infty), i \mathbb{R}\right) \oplus \Gamma\left(W^{+}\right)\right)
$$

where $\left(\mathbb{A}_{\alpha}, \Psi_{\alpha}\right)$ is a smooth pair consisting of a $U(1)$-connection on $\operatorname{det}(\mathfrak{s})$ and a section of $W^{+}$on $X_{+}(\infty)$, which extends $\left(A_{\alpha}, \psi_{\alpha}\right)$ on $[-2, \infty) \times Y$ (as a constant pair representing $\left.\Gamma_{\alpha} \in \mathcal{M}_{Y, X_{+}}\left(Y, \mathfrak{s}_{+}\right)\right)$. The corresponding gauge group is the $L_{3, \delta}^{2}$-gauge transformation group.

The following proposition describes the structure of $\mathcal{M}_{X_{+}}\left(\mathfrak{s}_{+}, \Gamma_{\alpha}\right)$. It is a smooth oriented manifold with dimension given by the Fredholm index of an operator $\mathcal{D}_{x}$ labelled by an element $x \in \mathcal{M}_{X_{+}}\left(\mathfrak{s}_{+}, \Gamma_{\alpha}\right)$ representing a solution $(\mathbb{A}, \Psi)$ to the perturbed Seiberg-Witten equations (49). The definition of

$$
\mathcal{D}_{x}: L_{2, \delta}^{2}\left(\Omega^{1}\left(X_{+}(\infty), i \mathbb{R}\right) \oplus \Gamma\left(W^{+}\right)\right) \longrightarrow L_{1, \delta}^{2}\left(\Omega^{0}\left(X_{+}(\infty), i \mathbb{R}\right) \oplus \Omega^{2,+}\left(X_{+}(\infty), i \mathbb{R}\right) \oplus \Gamma\left(W^{-}\right)\right)
$$

is

$$
\mathcal{D}_{x}(b, \Phi)=\left\{\begin{array}{l}
G_{(\mathbb{A}, \Psi)}^{*}(b, \Phi) \\
d^{+} b-2 q(\Psi, \Phi)-\mathfrak{p}_{\mathbb{A}}^{+}(b, \Phi) \\
\not D_{\mathbb{A}} \Phi+\frac{1}{2} b . \Psi+\mathfrak{p}_{\Psi}(b, \Phi) .
\end{array}\right.
$$

where $G_{(\mathbb{A}, \Psi)}^{*}$ is the adjoint of the linearization of the gauge group action in $L_{\delta}^{2}$-norm, the terms involving $\mathfrak{p}_{\mathbb{A}}^{+}$and $\mathfrak{p}_{\Psi}$ come from the linearization of the perturbation term in (49) supported in $[-2, \infty) \times Y$. Note that $\mathcal{D}_{x}$ is indeed a Fredholm operator, whose index is given by the Atiyah-Patodi-Singer index formula:

$$
\mathfrak{i}_{X_{+}}\left(\Gamma_{\alpha}\right)=\frac{1}{4}\left(\frac{-1}{4 \pi^{2}} \int_{X_{+}(\infty)} F_{\mathbb{A}} \wedge F_{\mathbb{A}}-2 \chi\left(X_{+}\right)-3 \sigma\left(X_{+}\right)\right)+\frac{1}{2} \rho\left(\Gamma_{\alpha}\right),
$$


where $\rho\left(\Gamma_{\alpha}\right)$ is the rho-invariant of the boundary operator $\mathcal{T}_{\left(A_{\alpha}, \psi_{\alpha}\right)}$ (Cf. the extended Hessian operator (13)). Note that $\frac{-1}{4 \pi^{2}} \int_{X_{+}(\infty)} F_{\mathbb{A}} \wedge F_{\mathbb{A}}$ is finite for any finite energy solution (Cf. Corollary 3.15)), and $\sigma\left(X_{+}\right)$is the signature of the intersection form on $H^{2}\left(X_{+}(0), Y ; \mathbb{R}\right)$ and $\chi\left(X_{+}\right)$is $2-2 b_{1}+b_{2}$ with $b_{1}=\operatorname{dim} H^{1}\left(X_{+}(0), Y ; \mathbb{R}\right)$ and $b_{2}=\operatorname{dim}^{2}\left(X_{+}(0), Y ; \mathbb{R}\right)$.

Proposition 4.2. Fix an open set $U \subset X_{+}(-2)$ away from the boundary of $X_{+}(-2)$. There is a Baire set of smooth imaginary-valued 2-forms with compact support in $U$ such that for any $\Gamma_{\alpha} \in \mathcal{M}_{Y, X_{+}}(\mathfrak{t}, \eta)$ : if $\mathfrak{i}_{X_{+}}\left(\Gamma_{\alpha}\right)<0$, then $\mathcal{M}_{X_{+}}\left(\mathfrak{s}_{+}, \Gamma_{\alpha}\right)$ is empty while if $\mathfrak{i}_{X_{+}}\left(\Gamma_{\alpha}\right) \geq 0$ and $\mathcal{M}_{X_{+}}\left(\mathfrak{s}_{+}, \Gamma_{\alpha}\right)$ is non-empty, then $\mathcal{M}_{X_{+}}\left(\mathfrak{s}_{+}, \Gamma_{\alpha}\right)$ is a smooth manifold of dimension $\mathfrak{i}_{X_{+}}\left(\Gamma_{\alpha}\right)$ oriented with a choice of orientation for the line $\Lambda^{\text {top }} H^{1}\left(X_{+}(0), Y ; \mathbb{R}\right) \otimes \Lambda^{\text {top }} H^{2,+}\left(X_{+}(0), Y ; \mathbb{R}\right)$. Moreover, the cokernel of $\mathcal{D}_{x}$ is trivial for any $x \in \mathcal{M}_{X_{+}}\left(\mathfrak{s}_{+}, \Gamma_{\alpha}\right)$.

The proof of this proposition is fairly standard, we omit the details here, see Proposition 2.14 [20], Proposition 8.5 and Corollary 9.2 in [25], Proposition 2.2 and Proposition 2.3 [33].

Remark 4.3. From the expression for the dimension formula (54), we know that if $\Gamma_{\alpha}$ and $\Gamma_{\alpha}^{\prime}$ are two different points in $\pi^{-1}(\alpha)$, there is a gauge transformation $u$ on $\operatorname{det}(\mathfrak{t})$ whose associated cohomology class $[u]$ is non-zero in $H^{1}(Y, \mathbb{Z}) / \operatorname{Im}\left(i_{+}^{*}\right)$, such that $u\left(\Gamma_{\alpha}\right)=\Gamma_{\alpha}^{\prime}$ in $\pi_{+}^{-1}(\alpha)$. Then

$$
\mathfrak{i}_{X_{+}}\left(\Gamma_{\alpha}\right)-\mathfrak{i}_{X_{+}}\left(\Gamma_{\alpha}^{\prime}\right)=<[u] \wedge c_{1}(\mathfrak{t}),[Y]>
$$

Note that if $[u] \in \operatorname{Ker}\left(c_{1}(\mathfrak{t})\right)$, then $<[u] \wedge c_{1}(\mathfrak{t}),[Y]>=0$. The relative grading on $\mathcal{M}_{Y}(\mathfrak{t}, \eta)$ defined in Definition 3.5 is related to $\mathfrak{i}_{X_{+}}$in the following way:

$$
\mathfrak{i}(\alpha, \beta)=\mathfrak{i}_{X_{+}}\left(\Gamma_{\beta}\right)-\mathfrak{i}_{X_{+}}\left(\Gamma_{\alpha}\right)(\bmod d(\mathfrak{t})),
$$

where $\Gamma_{\alpha} \in \pi_{+}^{-1}(\alpha)$ and $\Gamma_{\beta} \in \pi_{+}^{-1}(\beta)$.

Under the smoothness condition in Proposition 4.2, we now study the compactness of various components of the moduli space $\mathcal{M}_{X_{+}}\left(\mathfrak{s}_{+}\right)$. It is convenient to assemble the components in $\mathcal{M}_{X_{+}}\left(\mathfrak{s}_{+}\right)$with fixed dimension $d \geq 0$ as follows

$$
\mathcal{M}_{X_{+}}^{d}\left(\mathfrak{s}_{+}\right)=\bigcup_{\alpha \in \mathcal{M}_{Y}(\mathfrak{t}, \eta)}\left(\bigcup_{\Gamma_{\alpha} \in \pi_{+}^{-1}(\alpha), \mathfrak{i}_{X_{+}}\left(\Gamma_{\alpha}\right)=d} \mathcal{M}_{X_{+}}\left(\mathfrak{s}_{+}, \Gamma_{\alpha}\right)\right) .
$$

Then $\mathcal{M}_{X_{+}}\left(\mathfrak{s}_{+}\right)=\cup_{d \geq 0} \mathcal{M}_{X_{+}}^{d}\left(\mathfrak{s}_{+}\right)$, each $\mathcal{M}_{X_{+}}^{d}\left(\mathfrak{s}_{+}\right)$is called a stratum of dimension $d$ in $\mathcal{M}_{X_{+}}\left(\mathfrak{s}_{+}\right)$.

The next proposition establishes the required compactification for each stratum of dimension $d$ in $\mathcal{M}_{X_{+}}\left(\mathfrak{s}_{+}\right)$.

Proposition 4.4. For any $d \geq 0$, and each $\alpha \in \mathcal{M}_{Y}(\mathfrak{t}, \eta)$, there are only finitely many $\Gamma_{\alpha}$ in $\pi_{+}^{-1}(\alpha)$ with non-empty moduli space $\mathcal{M}_{X_{+}}\left(\mathfrak{s}_{+}, \Gamma_{\alpha}\right)$ in $\mathcal{M}_{X_{+}}^{d}\left(\mathfrak{s}_{+}\right)$. Moreover, there exists a 
compactification of $\mathcal{M}_{X_{+}}^{d}\left(\mathfrak{s}_{+}\right)$, denoted by $\overline{\mathcal{M}_{X_{+}}^{d}\left(\mathfrak{s}_{+}\right)}$, which is a smooth manifold with corners. The codimension $k$ boundary faces can be expressed in the following form

$$
\bigcup_{\left\{\alpha_{1}, \cdots, \alpha_{k}\right\}}\left(\bigcup_{\left\{\Gamma_{\alpha_{1}}, \cdots, \Gamma_{\alpha_{k}}\right\}} \mathcal{M}_{X_{+}}\left(\mathfrak{s}_{+}, \Gamma_{\alpha_{0}}\right) \times \hat{\mathcal{M}}_{\mathbb{R} \times Y}\left(\Gamma_{\alpha_{0}}, \Gamma_{\alpha_{1}}\right) \times \cdots \times \hat{\mathcal{M}}_{\mathbb{R} \times Y}\left(\Gamma_{\alpha_{k-1}}, \Gamma_{\alpha_{k}}\right)\right) .
$$

Here $\left\{\alpha_{1}, \cdots, \alpha_{k}\right\} \subset \mathcal{M}_{Y}(\mathfrak{t}, \eta)$ and $\Gamma_{\alpha_{i}} \in \pi_{+}^{-1}\left(\alpha_{i}\right)$ such that $\mathcal{M}_{X_{+}}\left(\mathfrak{s}_{+}, \Gamma_{\alpha_{i}}\right)$ is non-empty for each $0 \leq i \leq k$,

$$
d=\mathfrak{i}_{X_{+}}\left(\Gamma_{\alpha_{k}}\right)>\mathfrak{i}_{X_{+}}\left(\Gamma_{\alpha_{k-1}}\right)>\cdots>\mathfrak{i}_{X_{+}}\left(\Gamma_{\alpha_{1}}\right)>\mathfrak{i}_{X_{+}}\left(\Gamma_{\alpha_{0}}\right) \geq 0
$$

and $\hat{\mathcal{M}}_{\mathbb{R} \times Y}\left(\Gamma_{\alpha_{i}}, \Gamma_{\alpha_{i-1}}\right)$ is the moduli space of unparametrized flowlines for $\mathcal{C}_{\eta, \mathfrak{p}}$, connecting $\Gamma_{\alpha_{i}}$ and $\Gamma_{\alpha_{i-1}}$ on the configuration space $\mathcal{A}_{Y}$ modulo those gauge transformations which can be extended to $X_{+}$.

Proof. The proof is standard in Floer gauge theory, we only give a sketch here, following the techniques developed in section 4 of [20].

There is a constant $E$ depending only on the Riemannian metric on $X_{+}(\infty)$ such that for any finite energy solution $(\mathbb{A}, \Psi)$ in temporal gauge when restricted to $[-2, \infty) \times Y$, representing a point in $\mathcal{M}_{X_{+}}^{d}\left(\mathfrak{s}_{+}\right)$, we have $|\Psi(x)| \leq E$ for any $x \in X_{+}(\infty)$. This follows from the Weitzenböck formula for the Dirac operator $D_{\mathbb{A}}$ and the fact that, over the end, $(\mathbb{A}, \Psi)$ satisfies the asymptotic estimate of Proposition 4.1.

With this pointwise uniform bound on $\Psi$ and the asymptotic estimate in Proposition 4.1 , there is a uniform bound on the $L^{2}$-norm of $F_{\mathbb{A}}^{+}$so that

$$
-\frac{1}{4 \pi^{2}} \int_{X_{+}(\infty)} F_{\mathbb{A}} \wedge F_{\mathbb{A}}=\frac{1}{4 \pi^{2}} \int_{X_{+}(\infty)}\left(\left|F_{\mathbb{A}}^{+}\right|^{2}-\left|F_{\mathbb{A}}^{-}\right|^{2}\right) d v o l_{X_{+}}
$$

is uniformly bounded. This implies that there is a uniform upper bound on the $L^{2}$-norm of $F_{\AA}$.

Now let $\left(\mathbb{A}_{i}, \Psi_{i}\right)$ represent a sequence of elements in $\mathcal{M}_{X_{+}}^{d}\left(\mathfrak{s}_{+}\right)$. Then the standard bootstrapping arguments in elliptic regularity theory show that on any compact set of $X_{+}(\infty)$, there exists a subsequence of $\left(\mathbb{A}_{i}, \Psi_{i}\right)$ which, after suitable gauge transformations, converges in $C^{\infty}$-topology to a finite energy solution to the perturbed Seiberg-Witten equations (49) on the corresponding compact set of $X_{+}(\infty)$. These procedures, as illustrated in Theorem 4.1 [20], will produce an element in

$$
\bigcup_{\left\{\alpha_{1}, \cdots, \alpha_{k}\right\}}\left(\bigcup_{\left\{\Gamma_{\alpha_{1}}, \cdots, \Gamma_{\alpha_{k}}\right\}} \mathcal{M}_{X_{+}}\left(\mathfrak{s}_{+}, \Gamma_{\alpha_{0}}\right) \times \hat{\mathcal{M}}_{\mathbb{R} \times Y}\left(\Gamma_{\alpha_{0}}, \Gamma_{\alpha_{1}}\right) \times \cdots \times \hat{\mathcal{M}}_{\mathbb{R} \times Y}\left(\Gamma_{\alpha_{k-1}},\left(\Gamma_{\alpha_{k}}\right)\right)\right.
$$

with $\left\{\alpha_{1}, \cdots, \alpha_{k}\right\} \subset \mathcal{M}_{Y}(\mathfrak{t}, \eta)$ and $\Gamma_{\alpha_{i}} \in \pi_{+}^{-1}\left(\alpha_{i}\right)$ such that for each $0 \leq i \leq k$

$$
d=\mathfrak{i}_{X_{+}}\left(\Gamma_{\alpha_{k}}\right)>\mathfrak{i}_{X_{+}}\left(\Gamma_{\alpha_{k-1}}\right)>\cdots>\mathfrak{i}_{X_{+}}\left(\Gamma_{\alpha_{1}}\right)>\mathfrak{i}_{X_{+}}\left(\Gamma_{\alpha_{0}}\right) \geq 0 .
$$


An easy consequence of this convergence argument is that there are only finitely many $\Gamma_{\alpha} \in \pi_{+}^{-1}(\alpha)$ with non-empty $\mathcal{M}_{X_{+}}\left(\mathfrak{s}_{+}, \Gamma_{\alpha}\right)$ in $\mathcal{M}_{X_{+}}^{d}\left(\mathfrak{s}_{+}, \Gamma_{\alpha}\right)$.

Next we need to show that any element in (56) can glued together to produce an element in $\mathcal{M}_{X_{+}}\left(\mathfrak{s}_{+}, \Gamma_{\alpha_{k}}\right)$. This is the multiple gluing theorem in Theorem 4.23 and Proposition 4.25 of [20]. This implies that for any $\Gamma_{\alpha_{i}}$ appearing in (56), $\mathcal{M}_{X_{+}}\left(\mathfrak{s}_{+}, \Gamma_{\alpha_{i}}\right)$ is non-empty. Note that by the generic choices of various perturbations, all the moduli spaces in our discussion are smooth and the cokernels of the corresponding Fredholm operators are trivial, hence there is no obstruction in the multiple gluing theorem in Theorem 4.9 and Proposition 4.25 of [20].

The smooth structure with corners on the compactification can be obtained with the help of Lemma 4.26 in 20, we omit the details.

We should remark that there is a family version of Proposition 4.2 and Proposition 4.4, which will be crucial in the proof of the topological invariance of the various relative invariants introduced in the next Subsection.

For simplicity, we assume that the Riemannian metric on $Y$, the perturbation $\eta$ for the non-degeneracy of $\mathcal{M}_{Y}(\mathfrak{s}, \eta)$ and the perturbation $\mathfrak{p}$ for the smoothness of the moduli spaces of flowlines for $\mathcal{C}_{\eta, \mathfrak{p}}$ are fixed.

Let $g_{0}$ and $g_{1}$ be two Riemannian metrics on $X_{+}(\infty)$ which agree with the product metric $d t^{2}+g_{Y}$ over the end $[-2, \infty) \times Y$. Let $\omega_{0}$ and $\omega_{1}$ be two compactly supported self-dual forms, with respect to $g_{0}$ and $g_{1}$ respectively, in the Baire set in Proposition 4.2 such that the claims in Proposition 4.2 and Proposition 4.4 hold for the moduli spaces $\mathcal{M}_{X_{+}}\left(\mathfrak{s}_{+}, g_{0}, \omega_{0}\right)$ and $\mathcal{M}_{X_{+}}\left(\mathfrak{s}_{+}, g_{1}, \omega_{1}\right)$.

Proposition 4.5. There is a continuous interpolating family of metrics $g_{t}$ which agree with the product metric $d t^{2}+g_{Y}$ over the end $[-2, \infty) \times Y$ and compactly supported self-dual forms $\omega_{t}$ such that, for any $\Gamma_{\alpha} \in \mathcal{M}_{Y, X_{+}}(\mathfrak{t}, \eta)$ with $\mathfrak{i}_{X_{+}}\left(\Gamma_{\alpha}\right)=d \geq 0$, the family of moduli spaces

$$
\mathcal{M}_{d}^{F}\left(\mathfrak{s}_{+}, \Gamma_{\alpha}\right)=\bigcup_{t \in[0, t]} \mathcal{M}_{X_{+}}^{d}\left(\mathfrak{s}_{+}, \Gamma_{\alpha}, g_{t}, \omega_{t}\right)
$$

satisfies the following properties:

1. $\mathcal{M}_{d}^{F}\left(\Gamma_{\alpha}\right)$ is a $(d+1)$-dimensional, oriented, smooth manifold and the parameter projection $\mathcal{M}_{d}^{F}\left(\Gamma_{\alpha}\right) \rightarrow[0,1]$ is smooth and admits a product structure near the ends of $[0,1]$. The orientation on $\mathcal{M}_{d}^{F}\left(\Gamma_{\alpha}\right)$ is compatible with the orientations of $\mathcal{M}_{X_{+}}\left(\mathfrak{s}_{+}, \Gamma_{\alpha}, g_{0}, \omega_{0}\right)$ and $\mathcal{M}_{X_{+}}\left(\mathfrak{s}_{+}, \Gamma_{\alpha}, g_{1}, \omega_{1}\right)$ as the boundary.

2. There is a compactification of $\mathcal{M}_{d}^{F}\left(\Gamma_{\alpha}\right)$ which is compatible with the compactifications of $\mathcal{M}_{X_{+}}\left(\mathfrak{s}_{+}, \Gamma_{\alpha}, g_{0}, \omega_{0}\right)$ and $\mathcal{M}_{X_{+}}\left(\mathfrak{s}_{+}, \Gamma_{\alpha}, g_{1}, \omega_{1}\right)$ described in Proposition 4.4. In particular, 
the codimension one boundary of the compactification of $\mathcal{M}_{d}^{F}\left(\Gamma_{\alpha}\right)$ is given by

$$
\begin{gathered}
\mathcal{M}_{X_{+}}\left(\mathfrak{s}_{+}, \Gamma_{\alpha}, g_{0}, \omega_{0}\right) \cup\left(-\mathcal{M}_{X_{+}}\left(\mathfrak{s}_{+}, \Gamma_{\alpha}, g_{1}, \omega_{1}\right)\right) \\
\cup \bigcup_{t \in[0,1]}\left(\bigcup_{\alpha_{0} \in \mathcal{M}_{Y}(\mathfrak{t}, \eta)} \bigcup_{\Gamma_{\alpha_{0}} \in \pi_{+}^{-1}\left(\alpha_{0}\right)} M_{X_{+}}\left(\mathfrak{s}_{+}, \Gamma_{\alpha_{0}}, g_{t}, \omega_{t}\right) \times \hat{\mathcal{M}}_{\mathbb{R} \times Y}\left(\Gamma_{\alpha_{0}}, \Gamma_{\alpha}\right)\right) .
\end{gathered}
$$

Here $\Gamma_{\alpha_{0}} \in \pi_{+}^{-1}\left(\alpha_{0}\right)$ and $0 \leq \mathfrak{i}_{X_{+}}\left(\Gamma_{\alpha_{0}}\right)<d$.

The proof of this proposition is just the family version of arguments in the proofs of Proposition 4.2 and Proposition 4.4. We will not repeat the arguments here and leave the details to the reader.

\subsection{Relative invariants for 4-manifolds with boundary}

Let $X_{+}(0)$ be an oriented 4-manifold with one boundary $Y$. We endow $X_{+}(0)$ with a complete metric, denoted by $X_{+}(\infty)$, so that $X_{+}(\infty)$ has a cylindrical end modelled on $[-2, \infty) \times Y$. Let $\mathfrak{s}_{+}$be a $\operatorname{Spin}^{c}$ structure on $X_{+}(\infty)$ whose induced $\operatorname{Spin}^{c}$ structure on $Y$ is $\mathfrak{t}$ such that $c_{1}(\mathfrak{t})$ is non-torsion. There is a subgroup $\operatorname{Im}\left(i_{+}^{*}\right)$ of $\operatorname{Ker}\left(c_{1}(\mathfrak{t})\right)$ such that the covering map $\mathcal{M}_{Y, X_{+}}(\mathfrak{t}, \eta) \rightarrow \mathcal{M}_{Y}(\mathfrak{t}, \eta)$ has as its fiber an $H^{1}(Y, \mathbb{Z}) / \operatorname{Im}\left(i_{+}^{*}\right)$-homogeneous space.

Fix an orientation for the line $\Lambda^{t o p} H^{1}\left(X_{+}(0), Y ; \mathbb{R}\right) \otimes \Lambda^{t o p} H^{2,+}\left(X_{+}(0), Y ; \mathbb{R}\right)$. As studied in the previous Section, the finite energy moduli space has a well-defined asymptotic limit map:

$$
\mathcal{M}_{X_{+}}\left(\mathfrak{s}_{+}\right) \rightarrow \mathcal{M}_{Y, X_{+}}(\mathfrak{t}, \eta)
$$

The structure of $\mathcal{M}_{X_{+}}\left(\mathfrak{s}_{+}\right)$was established in Proposition 1.2 and Proposition 4.4 .

Denote by $\mathbb{A}\left(X_{+}\right)=\operatorname{Sym}^{*}\left(H_{0}\left(X_{+}\right)\right) \otimes \Lambda^{*}\left(H_{1}\left(X_{1}\right) /\right.$ Tor sion $)$ the free graded algebra generated by the class of the element in $H_{0}\left(X_{+}\right)$and the 1-cycle $\gamma \in H_{1}\left(X_{+}\right)$with degrees 2 and 1 respectively. Then the relative Seiberg-Witten invariant for $\left(X_{+}(\infty), \mathfrak{s}_{+}\right)$will be defined as a map

$$
S W_{X_{+}}(\mathfrak{s}, \cdot): \quad \mathbb{A}\left(X_{+}\right) \mapsto H F_{*,\left[\operatorname{Im}\left(i_{+}^{*}\right)\right]}^{S W}(Y, \mathfrak{t}) .
$$

For any monomial $z=U^{k} \gamma_{1} \wedge \gamma_{2} \wedge \cdots \wedge \gamma_{l}$ in $\mathbb{A}\left(X_{+}\right)$with $2 k+l=d \geq 0$, we need to consider all the components of dimension $d, \mathcal{M}_{X_{+}}^{d}\left(\mathfrak{s}_{+}\right)$in $\mathcal{M}_{X_{+}}\left(\mathfrak{s}_{+}\right)$. Recall that

$$
\mathcal{M}_{X_{+}}^{d}\left(\mathfrak{s}_{+}\right)=\bigcup_{\alpha \in \mathcal{M}_{Y}(\mathfrak{t}, \eta)}\left(\bigcup_{\Gamma_{\alpha} \in \pi_{+}^{-1}(\alpha), \mathfrak{i}_{X_{+}}\left(\Gamma_{\alpha}\right)=d} \mathcal{M}_{X_{+}}\left(\mathfrak{s}_{+}, \Gamma_{\alpha}\right)\right) .
$$

Choose $k$-points $\left\{x_{1}, \cdots, x_{k}\right\}$ in $X_{+}(\infty)$, and for each $x_{i}$, choose a complex line $L_{x_{i}}$ in $W_{x_{i}}^{+}$(the fiber of $W^{+}$over $x_{i}$ ). Also choose l-loops $\Xi=\left\{\pi_{1}, \cdots, \pi_{l}\right\}$ representing 1-cycles $\left\{\gamma_{1}, \cdots, \gamma_{l}\right\}$. For a generic choice of $\Lambda=\left\{\left(x_{1}, L_{x_{1}}\right), \cdots,\left(x_{k}, L_{x_{k}}\right)\right\}$, as a subset of $\mathcal{M}_{X_{+}}\left(\mathfrak{s}_{+}, \Gamma_{\alpha}\right)$ (for $\Gamma_{\alpha}$ with $i n d_{X_{+}}\left(\Gamma_{\alpha}\right)=d$ ),

$$
\mathcal{M}_{X_{+}}^{\Lambda}\left(\mathfrak{s}_{+}, \Gamma_{\alpha}\right)=\left\{[\mathbb{A}, \Psi] \in \mathcal{M}_{X_{+}}\left(\mathfrak{s}_{+}, \Gamma_{\alpha}\right) \mid \Psi\left(x_{i}\right) \in L_{x_{i}}, \text { for each } x_{i}, 1 \leq i \leq k\right\}
$$


is a $l$-dimensional, oriented and smooth submanifold of $\mathcal{M}_{X_{+}}\left(\mathfrak{s}_{+}, \Gamma_{\alpha}\right)$. Moreover, $M_{X_{+}}^{\Lambda}\left(\mathfrak{s}_{+}, \Gamma_{\alpha}\right)$ meets with each boundary face of the compactification $\overline{\mathcal{M}_{X_{+}}\left(\mathfrak{s}_{+}, \Gamma_{\alpha}\right)}$ transversally. This is the consequence of Proposition 4.2 and Proposition 4.4 and the standard application of Sard theorem.

Similarly, for any $l$-loops $\Xi=\left\{\pi_{1}, \cdots, \pi_{l}\right\}$, there is a holonomy map

$$
h_{\Xi}: \quad \mathcal{M}_{X_{+}}\left(\mathfrak{s}_{+}, \Gamma_{\alpha}\right) \longrightarrow U(1)^{l}
$$

given by the holonomies of $[\mathbb{A}, \Psi] \in \mathcal{M}_{X_{+}}\left(\mathfrak{s}_{+}, \Gamma_{\alpha}\right)$ along loops in $\Xi$. Then for a generic point $q \in U(1)^{l}$, there is a $2 k$-dimensional, oriented and smooth submanifold $\mathcal{M}_{X_{+}}^{\Xi}\left(\mathfrak{s}_{+}, \Gamma_{\alpha}\right)=h_{\Xi}^{-1}(q)$ of $\mathcal{M}_{X_{+}}\left(\mathfrak{s}_{+}, \Gamma_{\alpha}\right)$. Moreover, $\left.\mathcal{M}_{X_{+}}^{\Xi} \mathfrak{s}_{+}, \Gamma_{\alpha}\right)$ meets with each boundary face of the compactification $\overline{\mathcal{M}_{X_{+}}\left(\mathfrak{s}_{+}, \Gamma_{\alpha}\right)}$ transversally.

Then we obtain that

$$
\mathcal{M}_{X_{+}}^{\Lambda, \Xi}\left(\mathfrak{s}_{+}, \Gamma_{\alpha}\right)=\mathcal{M}_{X_{+}}^{\Lambda}\left(\mathfrak{s}_{+}, \Gamma_{\alpha}\right) \cap \mathcal{M}_{X_{+}}^{\Xi}\left(\mathfrak{s}_{+}, \Gamma_{\alpha}\right)
$$

is a compact, 0-dimensional, oriented and smooth manifold. We write

$$
S W_{X_{+}}\left(\mathfrak{s}_{+}, z, \Gamma_{\alpha}\right)=\#\left(\mathcal{M}_{X_{+}}^{\Lambda, \Xi}\left(\mathfrak{s}_{+}, \Gamma_{\alpha}\right)\right),
$$

an integer which is independent of the choices of $\Lambda$ and $\Xi$, as the space of $\{\Lambda, \Xi\}$ is pathconnected.

For those $\Gamma_{\alpha}$ with $\mathfrak{i}_{X_{+}}\left(\Gamma_{\alpha}\right) \neq d$, we set $S W_{X_{+}}\left(\mathfrak{s}_{+}, z, \Gamma_{\alpha}\right)=0$. Now we can define the following chain element in $C_{d,\left[\operatorname{Im}\left(i_{+}^{*}\right)\right]}^{S W}(Y, \mathfrak{t})$ (for $\left.d=\operatorname{deg}(z)\right)$ :

$$
S W_{X_{+}}\left(\mathfrak{s}_{+}, z\right)=\sum_{\alpha \in \mathcal{M}_{Y}(\mathfrak{t}, \eta)} \sum_{\Gamma_{\alpha}} S W_{X_{+}}\left(\mathfrak{s}_{+}, z, \Gamma_{\alpha}\right)<\Gamma_{\alpha}>
$$

which is a finite sum according to Proposition 4.4.

Proposition 4.6. $S W_{X_{+}}\left(\mathfrak{s}_{+}, z\right)$ is a cycle in $C_{d,\left[\operatorname{Im}\left(i_{+}^{*}\right)\right]}^{S W}(Y, \mathfrak{t})$ whose image in $H F_{d,\left[\operatorname{Im}\left(i_{+}^{*}\right)\right]}^{S W}(Y, \mathfrak{t})$ is independent of the metric and perturbation in the perturbed Seiberg-Witten equations (49).

Proof. To see that $S W_{X_{+}}\left(\mathfrak{s}_{+}, z\right)$ is a cycle we need to show that $\partial^{\operatorname{Im}\left(i_{+}^{*}\right)}\left(S W_{X_{+}}\left(\mathfrak{s}_{+}, z\right)\right)$ is zero. Consider the $(d+1)$-dimensional components, $\mathcal{M}_{X_{+}}^{d+1}\left(\mathfrak{s}_{+}\right)$, of $\mathcal{M}_{X_{+}}\left(\mathfrak{s}_{+}\right)$. Express

$$
M_{X_{+}}^{d+1}\left(\mathfrak{s}_{+}\right)=\cup_{\beta \in \mathcal{M}_{Y}(\mathfrak{t}, \eta)} \bigcup_{\Gamma_{\beta} \in \pi_{+}^{-1}(\beta), \mathfrak{i}_{X_{+}}\left(\Gamma_{\beta}\right)=d+1} \mathcal{M}_{X_{+}}\left(\mathfrak{s}_{+}, \Gamma_{\beta}\right),
$$

whose compactification is described in Proposition 4.4. Notice that the codimension one boundary face of $\overline{M_{X_{+}}^{d+1}\left(\mathfrak{s}_{+}\right)}$consists of

$$
\cup_{\alpha, \beta \in \mathcal{M}_{Y}(\mathfrak{t}, \eta)}\left(\bigcup_{\Gamma_{\alpha}, \Gamma_{\beta}} \mathcal{M}_{X_{+}}\left(\mathfrak{s}_{+}, \Gamma_{\alpha}\right) \times \hat{\mathcal{M}}_{\mathbb{R} \times Y}\left(\Gamma_{\alpha}, \Gamma_{\beta}\right)\right)
$$


where $\Gamma_{\alpha} \in \pi_{+}^{-1}(\alpha)$ and $\Gamma_{\beta} \in \pi_{+}^{-1}(\beta)$ with $\mathfrak{i}_{X_{+}}\left(\Gamma_{\beta}\right)=d+1$ and the unparametrized moduli space $\hat{\mathcal{M}}_{\mathbb{R} \times Y}\left(\Gamma_{\alpha}, \Gamma_{\beta}\right)$ is non-empty.

Choose $\Lambda$ and $\Xi$ away from the cylindrical end $[-2, \infty) \times Y$, then the submanifold

$$
\mathcal{M}_{X_{+}}^{\Lambda, \Xi}\left(\mathfrak{s}_{+}, \Gamma_{\beta}\right)=\mathcal{M}_{X_{+}}^{\Lambda}\left(\mathfrak{s}_{+}, \Gamma_{\beta}\right) \cap \mathcal{M}_{X_{+}}^{\Xi}\left(\mathfrak{s}_{+}, \Gamma_{\beta}\right)
$$

is a smooth oriented 1-manifold whose boundary consists of

$$
\cup_{\alpha \in \mathcal{M}_{Y}(\mathfrak{t}, \eta)} \bigcup_{\Gamma_{\alpha} \in \pi_{+}^{-1}(\alpha), \mathfrak{i}_{X_{+}}\left(\Gamma_{\alpha}\right)=d} \mathcal{M}_{X_{+}}^{\Lambda, \Xi}\left(\mathfrak{s}_{+}, \Gamma_{\alpha}\right) \times \hat{\mathcal{M}}_{\mathbb{R} \times Y}\left(\Gamma_{\alpha}, \Gamma_{\beta}\right) .
$$

The counting of boundary points with sign yields

$$
\sum_{\alpha \in \mathcal{M}_{Y}(\mathfrak{t}, \eta)} \sum_{\Gamma_{\alpha} \in \pi_{+}^{-1}(\alpha), \mathfrak{i}_{X_{+}}\left(\Gamma_{\alpha}\right)=d} S W_{X_{+}}\left(\mathfrak{s}_{+}, z, \Gamma_{\alpha}\right) \#\left(\hat{\mathcal{M}}_{\mathbb{R} \times Y}\left(\Gamma_{\alpha}, \Gamma_{\beta}\right)\right)=0
$$

Note that $\#\left(\hat{\mathcal{M}}_{\mathbb{R} \times Y}\left(\Gamma_{\alpha}, \Gamma_{\beta}\right)\right)$ is the coefficient of $\Gamma_{\beta}$ in $\partial^{\operatorname{Im}\left(i_{+}^{*}\right)}\left(\Gamma_{\alpha}\right)$, this shows that $\partial^{\operatorname{Im}\left(i_{+}^{*}\right)}\left(S W_{X_{+}}\left(\mathfrak{s}_{+}, z\right)\right)=0$.

To establish the independence of $\left[S W_{X_{+}}\left(\mathfrak{s}_{+}, z\right)\right]$, as an element in $\operatorname{HF}_{d,\left[\operatorname{Im}\left(i_{+}^{*}\right)\right]}^{S W}(Y, \mathfrak{t})$ on the choice of metric and perturbation on $X_{+}(\infty)$, we need to study the family version of moduli spaces interpolating between $\mathcal{M}_{X_{+}}\left(\mathfrak{s}_{+}, g_{0}, \omega_{0}\right)$ and $\mathcal{M}_{X_{+}}\left(\mathfrak{s}_{+}, g_{1}, \omega_{1}\right)$.

Choose $\Lambda$ and $\Xi$ away from the cylindrical end $[-2, \infty) \times Y$, then Proposition 4.5 implies that,

$$
\begin{gathered}
S W_{X_{+}}\left(\mathfrak{s}_{+}, z, g_{0}, \omega_{0}\right)-S W_{X_{+}}\left(\mathfrak{s}_{+}, z, g_{1}, \omega_{1}\right) \\
=\sum_{\alpha_{0}, \alpha \in \mathcal{M}_{Y}(\mathfrak{t}, \eta)} \sum_{\Gamma_{\alpha_{0}}, \Gamma_{\alpha}} H_{\Gamma_{\alpha_{0}}} n_{\Gamma_{\alpha_{0}} \Gamma_{\alpha}}<\Gamma_{\alpha}>,
\end{gathered}
$$

where $\Gamma_{\alpha_{0}} \in \pi_{+}^{-1}\left(\alpha_{0}\right)$ with $\mathfrak{i}_{X_{+}}\left(\Gamma_{\alpha_{0}}\right)=d-1$ and $\Gamma_{\alpha} \in \pi_{+}^{-1}(\alpha)$ with $\mathfrak{i}_{X_{+}}\left(\Gamma_{\alpha}\right)$, $n_{\Gamma_{\alpha} \Gamma_{\beta}}=\#\left(\hat{\mathcal{M}}_{\mathbb{R} \times Y}\left(\Gamma_{\alpha_{0}}, \Gamma_{\alpha}\right)\right)$, and $H_{\Gamma_{\alpha_{0}}}$ is the counting of the points $([\mathbb{A}, \Psi], t)$ in $\mathcal{M}_{d-1}^{F}\left(\Gamma_{\alpha_{0}}\right)=\cup_{t \in[0,1]} \mathcal{M}_{X_{+}}\left(\mathfrak{s}_{+}, \Gamma_{\alpha_{0}}, g_{t}, \omega_{t}\right)$ satisfying

1. $[\mathbb{A}, \Psi] \in \mathcal{M}_{X_{+}}\left(\mathfrak{s}_{+}, \Gamma_{\alpha_{0}}, g_{t}, \omega_{t}\right)$ such that $\Psi\left(x_{i}\right) \in L_{x_{i}}$ for each $\left(x_{i}, L_{x_{i}}\right) \in \Lambda$,

2. $h_{\Xi}([\mathbb{A}, \Psi])=q$ for a generic $q \in U(1)^{l}$.

Proposition 4.5 implies that $H_{\Gamma_{\alpha_{0}}}$ is well-defined, and

$$
\sum_{\alpha_{0}, \alpha \in \mathcal{M}_{Y}(\mathfrak{t}, \eta)} \sum_{\Gamma_{\alpha_{0}, \Gamma_{\alpha}}} H_{\Gamma_{\alpha_{0}}} n_{\Gamma_{\alpha_{0}} \Gamma_{\alpha}}<\Gamma_{\alpha}>
$$

is a boundary chain element, hence, $S W_{X_{+}}\left(\mathfrak{s}_{+}, z, g_{0}, \omega_{0}\right)$ and $S W_{X_{+}}\left(\mathfrak{s}_{+}, z, g_{1}, \omega_{1}\right)$ define the same element in $H F_{*,\left[\operatorname{Im}\left(i_{+}^{*}\right)\right]}^{S W}(Y, \mathfrak{t})$. 
To summarize, we have defined a relative Seiberg-Witten invariant as the following linear functional:

$$
\begin{aligned}
S W_{X_{+}}\left(\mathfrak{s}_{+}, \cdot\right): \quad \mathbb{A}\left(X_{+}\right) & \longrightarrow H F_{*,\left[\operatorname{Im}\left(i_{+}^{*}\right)\right]}^{S W}(Y, \mathfrak{t}) \\
z & \mapsto \quad\left[S W_{X_{+}}\left(\mathfrak{s}_{+}, z\right)\right] .
\end{aligned}
$$

$\left[S W_{X_{+}}\left(\mathfrak{s}_{+}, z\right)\right]$ has an representative written as in (57).

Notice that there is a homomorphism $\left(i_{+}\right)_{*}: \mathbb{A}(Y) \rightarrow \mathbb{A}(X)$, which induces an action of $\mathbb{A}(Y)$ on $\mathbb{A}(X)$. Then it is evident that from the definition of the action of $\mathbb{A}(Y)$ on $H F_{*,\left[\operatorname{Im}\left(i_{+}^{*}\right)\right]}^{S W}(Y, \mathfrak{t})$ and the definition of the relative invariant, $S W_{X_{+}}\left(\mathfrak{s}_{+}, \cdot\right)$ in $(58)$ is $\mathbb{A}(Y)$-equivariant, in the sense that,

$$
\left[S W_{X_{+}}\left(\mathfrak{s}_{+},\left(i_{+}\right)_{*}(\gamma) z\right)\right]=\gamma\left[S W_{X_{+}}\left(\mathfrak{s}_{+}, z\right)\right]
$$

for any $\gamma \in \mathbb{A}(Y)$ and $z \in \mathbb{A}(X)$.

Remark 4.7. The definition of the relative Seiberg-Witten invariant can be generalized to the case that $X_{+}$has several boundary components. For example, if $\partial\left(X_{+}\right)=\left(-Y_{1}\right) \cup Y_{2}$, let $\mathfrak{s}_{+}$ be a $\operatorname{Spin}^{c}$ structure on $X_{+}$whose restrictions to the boundary manifolds are non-torsion $\operatorname{Spin}^{c}$ structures $\mathfrak{t}_{1}$ and $\mathfrak{t}_{2}$ respectively. Let $i_{1}$ and $i_{2}$ be the boundary embedding map, $\operatorname{Im}\left(i_{1}^{*}\right)$ and $\operatorname{Im}\left(i_{2}^{*}\right)$ be the range of the induced maps on $H^{1}\left(X_{+}, \mathbb{Z}\right)$. Endowing $X_{+}$with a complete metric such that $X_{+}(\infty)$ is a cylindrical-end manifold modelled on $(-\infty, 2] \times Y_{1} \cup[2, \infty) \times Y_{2}$, then we can define a relative Seiberg-Witten invariant for $\left(X_{+}, s_{+}\right)$:

$$
S W_{X_{+}}\left(\mathfrak{s}_{+}, \cdot\right): \quad \mathbb{A}\left(X_{+}\right) \longrightarrow H F_{*,\left[\operatorname{Im}\left(i_{1}^{*}\right)\right]}^{S W}\left(-Y_{1},-\mathfrak{t}_{1}\right) \otimes H F_{*,\left[\operatorname{Im}\left(i_{2}^{*}\right)\right]}^{S W}\left(Y_{2}, \mathfrak{t}_{2}\right) .
$$

In other words, for any $z \in \mathbb{A}\left(X_{+}\right), \quad S W_{X_{+}}\left(\mathfrak{s}_{+}, z\right)$ defines a homomorphism from $H F_{*,\left[\operatorname{Im}\left(i_{1}^{*}\right)\right]}^{S W}\left(Y_{1}, \mathfrak{t}_{1}\right)$ to $H F_{*,\left[\operatorname{Im}\left(i_{2}^{*}\right)\right]}^{S W}\left(Y_{2}, \mathfrak{t}_{2}\right)$ using the natural pairing between $H F_{\left.*, \operatorname{Im}\left(i_{1}^{*}\right)\right]}^{S W}\left(Y_{1}, \mathfrak{t}_{1}\right)$ and $H F_{*,\left[\operatorname{Im}\left(i_{1}^{*}\right)\right]}^{S W}\left(-Y_{1},-\mathfrak{t}_{1}\right)$.

\subsection{Gluing formulae for the Seiberg-Witten invariants}

Consider a closed 4-manifold $(X, \mathfrak{s})$ with $b_{2}^{+} \geq 1$ and a $\operatorname{Spin}^{c}$ structure $\mathfrak{s}$. Suppose that $X$ has a decomposition $X_{+} \cup_{Y} X_{+}$along a 3-dimensional submanifold $Y$ where $b_{1}(Y)>0$ and $\mathfrak{t}=\left.\mathfrak{s}\right|_{Y}$ is non-torsion. In this subsection we will develop a gluing theorem for the moduli space of the Seiberg-Witten monopoles on $(X, \mathfrak{s})$ in terms of the the moduli spaces for the SeibergWitten monopoles on $\left(X_{ \pm}, \mathfrak{s}_{ \pm}\right)$with $\mathfrak{s}_{ \pm}=\left.\mathfrak{s}\right|_{X_{ \pm}}$. Let $i_{ \pm}$be the boundary embedding maps from $X_{ \pm} \rightarrow X$, and let $\operatorname{Im}\left(i_{ \pm}^{*}\right)$ be the range of the map $i_{ \pm}^{*}: H^{1}\left(X_{ \pm}, \mathbb{Z}\right) \rightarrow H^{1}(Y, \mathbb{Z})$.

The set of $\operatorname{Spin}^{c}$ structures on $X$ which agree with $\mathfrak{s}_{ \pm}$when restricted to $X_{ \pm}$is an affine space over $H^{1}(Y, \mathbb{Z}) /\left(\operatorname{Im}\left(i_{+}^{*}\right)+\operatorname{Im}\left(i_{-}^{*}\right)\right)$. Denote this set of $\operatorname{Spin}^{c}$ structures on $X$ by $\operatorname{Spin}^{c}\left(X, \mathfrak{s}_{ \pm}\right)$. 
Note that $\operatorname{Spin}^{c}\left(X, \mathfrak{s}_{ \pm}\right)$can be obtained by gluing the $\operatorname{Spin}^{c}$ structures $\mathfrak{s}_{ \pm}$on $X_{ \pm}$along $Y$ using gauge transformations on $(Y, \mathfrak{t})$. The equivalent classes of $\operatorname{Spin}^{c}$ structures, obtained in this way, are classified by the connected components of $H^{1}(Y, \mathbb{Z}) /\left(\operatorname{Im}\left(i_{+}^{*}\right)+\operatorname{Im}\left(i_{-}^{*}\right)\right)$. Denote by $\mathfrak{s}_{+} \#_{u} \mathfrak{s}_{-}$ the resulting $\operatorname{Spin}^{c}$ structure on $X$ obtained by gluing $\mathfrak{s}_{ \pm}$using the gauge transformation $u$, then

$$
\operatorname{Spin}^{c}\left(X, \mathfrak{s}_{ \pm}\right)=\left\{\mathfrak{s}_{+} \#{ }_{u} \mathfrak{s}_{-} \mid u \in \mathcal{G}_{Y},[u] \in H^{1}(Y, \mathbb{Z}) /\left(\operatorname{Im}\left(i_{+}^{*}\right)+\operatorname{Im}\left(i_{-}^{*}\right)\right)\right\}
$$

Consider a family of metrics $\left\{g_{R}\right\}_{R>0}$ on $X$ such that for each $X(R)=\left(X, g_{R}\right)$, there is an isometrically embedded submanifold $\left([-R-2, R+2] \times Y, d t^{2}+g_{Y}\right)$. There are two 4-manifolds $X_{ \pm}(R)$ obtained by $X(R)=X_{+}(R) \cup X_{-}(R)$. As $R \rightarrow \infty, X(R)$ has a geometric limit, which are 4-manifolds with cylindrical ends, denoted by $X_{ \pm}(\infty)$.

In the previous Subsection, we defined relative Seiberg-Witten invariants for $\left(X_{ \pm}(\infty), \mathfrak{s}_{ \pm}\right)$ using the finite energy moduli spaces of the perturbed Seiberg-Witten equations on $\left(X_{ \pm}(\infty), \mathfrak{s}_{ \pm}\right)$. These relative Seiberg-Witten invariants are $\mathbb{A}(Y)$-equivariant linear functionals:

$$
S W_{X_{ \pm}}\left(\mathfrak{s}_{ \pm}, \cdot\right): \quad \mathbb{A}\left(X_{ \pm}\right) \longrightarrow H F_{*,\left[m\left(i_{ \pm}^{*}\right)\right]}^{S W}( \pm Y, \pm \mathfrak{t}) .
$$

Fix an orientation on the line $\Lambda^{t o p} H^{1}(X, \mathbb{R}) \otimes \Lambda^{t o p} H^{2,+}(X, \mathbb{R})$ which is induced from tensoring the orientations on $\Lambda^{\text {top }} H^{1}\left(X_{+}(0), Y ; \mathbb{R}\right) \otimes \Lambda^{t o p} H^{2,+}\left(X_{+}(0), Y ; \mathbb{R}\right)$ and $\Lambda^{\text {top }} H^{1}\left(X_{-}(0), Y ; \mathbb{R}\right) \otimes \Lambda^{t o p} H^{2,+}\left(X_{-}(0), Y ; \mathbb{R}\right)$. When $b_{2}^{+}=1$, we also need to fix an orientation on the line $H^{2,+}(X, \mathbb{R})$ such that $c_{1}(\mathfrak{s}) \cdot \omega^{+}>0$ for an oriented generator $\omega^{+}$of $H^{2,+}(X, \mathbb{R})$. Then there is a Seiberg-Witten invariant as defined by Taubes in [34], which is a linear functional:

$$
S W_{X}(\mathfrak{s}, \cdot): \quad \mathbb{A}(X) \longrightarrow \mathbb{Z}
$$

where $\mathbb{A}(X)=\operatorname{Sym}^{*}\left(H_{0}(X, \mathbb{Z})\right) \otimes \Lambda^{*}\left(H_{1}(X, \mathbb{Z}) /\right.$ Torsion $)$ is the free graded algebra generated by the class of elements in $H_{0}(X, \mathbb{Z})$ and $H_{1}(X, \mathbb{Z})$ with degree 2 and 1 respectively.

On $(X(R), \mathfrak{s})$ for $\mathfrak{s} \in \operatorname{Spin}^{c}\left(X, \mathfrak{s}_{ \pm}\right)$, consider the following perturbed Seiberg-Witten equations for a pair consisting of a $U(1)$-connection $\mathbb{A}$ on $\operatorname{det}(\mathfrak{s})$ and a spinor section $\Psi$ in $W^{+}$:

$$
\left\{\begin{array}{l}
F_{\mathbb{A}}^{+}=q(\Psi, \Psi)+\omega^{+}+\omega^{-}+\chi\left(\eta^{+}+\mathfrak{p}_{\mathbb{A}}^{+}\right) \\
\not \mathbb{A}_{\mathbb{A}} \Psi=\chi \mathfrak{p}_{\Psi}
\end{array}\right.
$$

where we use the following notation.

- $\omega^{ \pm}$are imaginary valued self-dual 2-forms on $X_{ \pm}(0)$ with compact support in a non-empty open set such that Proposition 4.2 and Proposition 4.4 hold for the finite energy moduli spaces for $\left(X_{ \pm}(\infty), \mathfrak{s}_{ \pm}\right)$.

- $\left(\eta^{+}+\mathfrak{p}_{\mathbb{A}}^{+}, \mathfrak{p}_{\Psi}\right)$ is the perturbation of the Seiberg-Witten equations on $[-2, \infty) \times Y$ as written in (30) (where $\eta$ and $\mathfrak{p}$ satisfy Remark (3.4) and Condition (3.12)), and 
- $\chi$ is a cut-off function on $X(R)$ which is 1 on $[-R, R] \times Y$ and 0 on $X_{ \pm}(-2)$.

The moduli space of the Seiberg-Witten monopoles on $(X(R), \mathfrak{s})$, denoted by $\mathcal{M}_{X(R)}(\mathfrak{s})$, is the quotient of the set of smooth solutions to the above perturbed Seiberg-Witten equations (59) by the action of gauge group $C^{\infty}(X(R), U(1))$. Then (Cf. [15] [23]) for generic $\omega^{ \pm}, \mathcal{M}_{X(R)}(\mathfrak{s})$, if non-empty, is a compact, oriented and smooth manifold of dimension given by

$$
d_{X}(\mathfrak{s})=\frac{1}{4}\left(c_{1}(\mathfrak{s})^{2}-(2 \chi(X)+3 \sigma(X))\right) \geq 0 .
$$

Any solution $(\mathbb{A}, \Psi)$ to the perturbed Seiberg-Witten equations (59), when restricted to $[-R, R] \times Y$, is gauge equivalent to a path of gradient flowlines of $\mathcal{C}_{\eta, \mathfrak{p}}$ on $\mathcal{A}_{Y}$. Denote this associated path of flowlines by $\gamma(t)=(A(t), \psi(t))$, then the next lemma ensures that the variation of $\mathcal{C}_{\eta, \mathfrak{p}}$ along the associated path $\gamma(t)$ is uniformly bounded.

Lemma 4.8. There is a constant $E$ independent of $R$ such that for any solution $(\mathbb{A}, \Psi)$ to the perturbed Seiberg-Witten equations (59) on $(X(R), \mathfrak{s})$, the variation of $\mathcal{C}_{\eta, \mathfrak{p}}$ along the associated path of gradient flowlines $\gamma(t)=(A(t), \psi(t))$ is uniformly bounded by $E$, that is

$$
\left|\mathcal{C}_{\eta, \mathfrak{p}}(\gamma(-R))-\mathcal{C}_{\eta, \mathfrak{p}}(\gamma(R))\right| \leq E .
$$

Proof. From Lemma 3.14, we only need to show that $|\mathcal{C}(\gamma(-R))-\mathcal{C}(\gamma(R))|$ is uniformly bounded. Note that

$$
\begin{aligned}
0 & \leq \mathcal{C}(\gamma(-R))-\mathcal{C}(\gamma(R)) \\
& =\frac{1}{2} \int_{\left[R_{1}, R_{2}\right] \times Y} F_{\mathbb{A}} \wedge F_{\mathbb{A}}+\int_{\partial X_{+}(0)}<\psi, \not_{A} \psi>d v o l_{Y}-\int_{\partial X_{-}(0)}<\psi, \not_{A} \psi>d v o l_{Y} .
\end{aligned}
$$

A standard application of the Weitzenböck formulae implies that, there is a uniform pointwise bound for $|\Psi|$. Hence there is a uniform pointwise bound for $\left|F_{\mathbb{A}}^{+}\right|$independent of $R$ and $(\mathbb{A}, \Psi)$, from which, with the aid of the perturbed Seiberg-Witten equations, we obtain a uniform bound for

$$
\int_{\partial X_{+}(0)}<\psi, \not \partial_{A} \psi>d v o l_{Y} \quad \text { and } \int_{\partial X_{-}(0)}<\psi, \not \partial_{A} \psi>d v o l_{Y}
$$

Hence, there is a constant $C>0$ such that

$$
\frac{1}{2} \int_{[-R, R] \times Y} F_{\mathbb{A}} \wedge F_{\mathbb{A}} \geq-C .
$$

Notice that there is an identity

$$
\begin{aligned}
& \frac{1}{4 \pi^{2}}<c_{1}(\mathfrak{s})^{2},[X]> \\
= & -\int_{X(R)} F_{\mathbb{A}} \wedge F_{\mathbb{A}} \\
= & \left\|F_{\mathbb{A}}^{+}\right\|_{L^{2}\left(X_{+}(0) \cup X_{-}(0)\right)}^{2}-\left\|F_{\mathbb{A}}^{-}\right\|_{L^{2}\left(X_{+}(0) \cup X_{-}(0)\right)}^{2}-\int_{[-R, R] \times Y} F_{\mathbb{A}} \wedge F_{\mathbb{A}} .
\end{aligned}
$$


From (60) and (61), we know that $\left\|F_{\mathbb{A}}^{-}\right\|_{L^{2}\left(X_{+}(0) \cup X_{-}(0)\right)}^{2}$ is uniformly bounded, which in turn implies that

$$
\left|\frac{1}{2} \int_{[-R, R] \times Y} F_{\mathbb{A}} \wedge F_{\mathbb{A}}\right|
$$

is also uniformly bounded. This completes the proof of the lemma.

An immediate consequence of the above finite energy lemma concerning any solution to the perturbed Seiberg-Witten equations (59) is the following geometric limit theorem as $R \rightarrow \infty$.

Theorem 4.9. Let $\left(\mathbb{A}_{R}, \Psi_{R}\right)$ be a solution to the perturbed Seiberg-Witten equations (59) on $(X(R), \mathfrak{s})$ for $\mathfrak{s} \in \operatorname{Spin}^{c}\left(X, \mathfrak{s}_{ \pm}\right)$, then there is a finite integer $k \geq 0$ such that as $R \rightarrow \infty$, there exists a subsequence of $\left(\mathbb{A}_{R}, \Psi_{R}\right)$ which converges in $L_{2, \text { loc }}^{2}$-topology to

$$
\left\{\left(\mathbb{A}_{+}, \Psi_{+}\right),\left(\mathbb{A}_{1}, \Psi_{1}\right),\left(\mathbb{A}_{2}, \Psi_{2}\right), \cdots\left(\mathbb{A}_{k}, \Psi_{k}\right),\left(\mathbb{A}_{-}, \Psi_{-}\right)\right\}
$$

with compatible boundary asymptotic limits in the sense of section 6.2 in [24]. Here $\left(\mathbb{A}_{ \pm}, \Psi_{ \pm}\right)$ are solutions to the perturbed Seiberg-Witten equations $(49)$ on $\left(X_{ \pm}(\infty), \mathfrak{s}_{ \pm}\right)$, and $\left(\mathbb{A}_{i}, \Psi_{i}\right)^{\prime}$ s are solutions to the perturbed Seiberg-Witten equations (30) on $(\mathbb{R} \times Y, \mathfrak{t})$.

Proof. When restricted to $[-R, R] \times Y \subset X(R),\left(\mathbb{A}_{R}, \Psi_{R}\right)$ is gauge equivalent to a path $\gamma_{R}(t)=(A(t), \psi(t))\left(t \in[-R, R]\right.$ which is a gradient flowline of $\mathcal{C}_{\eta, \mathfrak{p}}$ on $\mathcal{A}_{Y}$ with uniformly bounded energy. Introduce the following function on $[-R, R]$

$$
f_{R}(t)=\| \nabla \mathcal{C}_{\eta, \mathfrak{p}}\left(\gamma_{R}(t) \|_{L^{2}(Y)}^{2}\right.
$$

Then $\int_{-R}^{R} f_{R}(t) d t$ is uniformly bounded. The argument in [15] can be employed here to show that as $R \rightarrow \infty$, there is at least one point $t_{0} \in \mathbb{R}$ such that the geometric limit of $f_{R}$ vanishes at $t_{0}$, hence the geometric limit of $\left(\mathbb{A}_{R}, \Psi_{R}\right)$ splits into two solutions to the perturbed SeibergWitten equations (49) on $\left(X_{ \pm}(\infty), \mathfrak{s}_{ \pm}\right)$. Following the procedures in the proof of Theorem 4.1 in [20], there exists a subsequence of $\left\{\left(\mathbb{A}_{R}, \Psi_{R}\right)\right\}_{R}$ which converges in $L_{2, l o c}^{2}$-topology to

$$
\left\{\left(\mathbb{A}_{+}, \Psi_{+}\right),\left(\mathbb{A}_{1}, \Psi_{1}\right),\left(\mathbb{A}_{2}, \Psi_{2}\right), \cdots\left(\mathbb{A}_{k}, \Psi_{k}\right),\left(\mathbb{A}_{-}, \Psi_{-}\right)\right\}
$$

with the properties as claimed in the theorem. The upper bound on $k$ follows from the uniformly bounded energy for $\left\{\left(\mathbb{A}_{R}, \Psi_{R}\right)\right\}_{R}$.

The standard gluing argument shows that any sequence of solutions with compatible asymptotic limits can be glued to produce a solution to the perturbed Seiberg-Witten equations (59) on $(X(R), \mathfrak{s})$ for a sufficiently large $R$ and some $\mathfrak{s} \in \operatorname{Spin}^{c}\left(X, \mathfrak{s}_{ \pm}\right)$. In order to formulate this 
gluing theorem, we briefly discuss various gluing models by studying the asymptotic limit maps for the moduli spaces on $\left(X_{ \pm}(\infty), \mathfrak{s}_{ \pm}\right)$.

The moduli spaces $\mathcal{M}_{X_{ \pm}}\left(\mathfrak{s}_{ \pm}\right)$are described in Proposition 4.2 and Proposition 4.4, in particular, there are two asymptotic limit maps:

$$
\partial_{\infty}^{ \pm}: \quad \mathcal{M}_{X_{ \pm}}\left(\mathfrak{s}_{ \pm}\right) \longrightarrow \mathcal{M}_{Y, X_{ \pm}}(\mathfrak{t}, \eta)
$$

where $\mathcal{M}_{Y, X_{ \pm}}(\mathfrak{t}, \eta)$ are covering spaces of $\mathcal{M}_{Y}(\mathfrak{t}, \eta)$ with fibers consisting of $H^{1}(Y, \mathbb{Z}) / \operatorname{Im}\left(i_{ \pm}^{*}\right)$ homogeneous spaces. Recall that $\mathcal{M}_{Y, X_{ \pm}}(\mathfrak{t}, \eta)$ and $\mathcal{M}_{Y, X}$ are the moduli spaces of 3-dimensional monopoles on $(Y, \mathfrak{t})$ modulo those gauge transformations which can be extended to $X_{ \pm}$and $X$ respectively.

Denote by $\mathcal{M}_{Y,+/-}$ the equivalent classes of 3 -dimensional monopoles on $(Y, \mathfrak{t})$ under the action of gauge transformations on $\operatorname{det}(\mathfrak{t})$ which can be extended to either $X_{+}$or $X_{-}$(not necessarily both). Then $\mathcal{M}_{Y,+/-}$ is a covering space of $\mathcal{M}_{Y}(\mathfrak{t}, \eta)$ with fiber a $H^{1}(Y, \mathbb{Z}) /\left(\operatorname{Im}\left(i_{+}^{*}\right)+\operatorname{Im}\left(i_{-}^{*}\right)\right)$ homogeneous space. Hence, there is an action of $H^{1}(Y, \mathbb{Z}) /\left(\operatorname{Im}\left(i_{+}^{*}\right)+\operatorname{Im}\left(i_{-}^{*}\right)\right)$ on $\mathcal{M}_{Y,+/-}$, this action defines an action of $H^{1}(Y, \mathbb{Z}) /\left(\operatorname{Im}\left(i_{+}^{*}\right)+\operatorname{Im}\left(i_{-}^{*}\right)\right)$ on $H F_{*,\left[\operatorname{Im}\left(i_{+}^{*}\right)+\operatorname{Im}\left(i_{-}^{*}\right)\right]}^{S W}(Y, \eta)$, that is, for any $[u] \in H^{1}(Y, \mathbb{Z}) /\left(\operatorname{Im}\left(i_{+}^{*}\right)+\operatorname{Im}\left(i_{-}^{*}\right)\right)$, there is an $\mathbb{A}(Y)$-equivariant homomorphism on $H F_{*,\left[\operatorname{Im}\left(i_{+}^{*}\right)+\operatorname{Im}\left(i_{-}^{*}\right)\right]}^{S W}(Y, \eta)$ which decreases degree in $H F_{*,\left[\operatorname{Im}\left(i_{+}^{*}\right)+\operatorname{Im}\left(i_{-}^{*}\right)\right]}(Y, \eta)$ by $<[u] \wedge c_{1}(\eta),[Y]>$.

The following commutative diagram illustrates the relationship between these covering spaces of $\mathcal{M}_{Y}(\mathfrak{t}, \eta)$ (the structure groups of covering spaces over $\mathcal{M}_{Y}(\mathfrak{t}, \eta)$ are given by Diagram (5) in the Introduction):

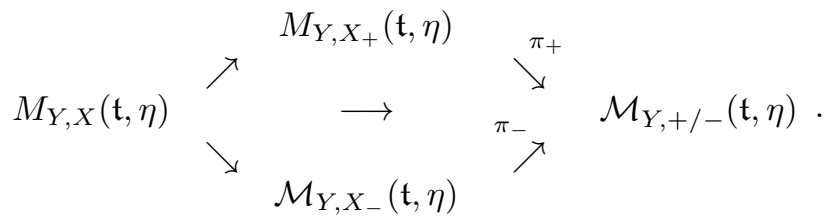

The compositions of $\pi_{ \pm}$with $\partial_{\infty}^{ \pm}$give rise to the following asymptotic limit maps:

$$
\pi_{ \pm} \circ \partial_{\infty}^{ \pm}: \quad \mathcal{M}_{X_{ \pm}}\left(\mathfrak{s}_{ \pm}\right) \longrightarrow \mathcal{M}_{Y,+/-}(\mathfrak{t}, \eta)
$$

Denote by $\mathcal{M}_{X_{ \pm}, X}\left(\mathfrak{s}_{ \pm}\right)$the moduli spaces of solutions to the perturbed Seiberg-Witten equations (49) on $\left(X_{ \pm}(\infty), \mathfrak{s}_{ \pm}\right)$modulo those gauge transformations on $\left(X_{ \pm}(\infty), \mathfrak{s}_{ \pm}\right)$which can be extended to $X$.

The following gluing theorem establishes that for a sufficiently large $R$, the moduli space $\mathcal{M}_{X(R)}(\mathfrak{s})$ for any $\mathfrak{s} \in \operatorname{Spin}^{c}\left(X, \mathfrak{s}_{ \pm}\right)$can be obtained by gluing certain components of the moduli spaces $\mathcal{M}_{X_{ \pm}}\left(\mathfrak{s}_{ \pm}\right)$on the cylindrical-end 4-manifolds $\left(X_{ \pm}(\infty), \mathfrak{s}_{ \pm}\right)$. 
Theorem 4.10. For any $\operatorname{Spin}^{c}$ structure $\mathfrak{s}=s_{+} \#{ }_{u} s_{-} \in \operatorname{Spin}^{c}\left(X, \mathfrak{s}_{ \pm}\right)$with u represents a gauge class in $H^{1}(Y, \mathbb{Z}) /\left(\operatorname{Im}\left(i_{+}^{*}\right)+\operatorname{Im}\left(i_{-}^{*}\right)\right)$, there is a sufficiently large $R_{0}$ such that for any $R>R_{0}$, there exists the following orientation preserving diffeomorphism

$$
\mathcal{M}_{X(R)}(\mathfrak{s}) \cong[u]\left(\mathcal{M}_{X_{+}}\left(\mathfrak{s}_{+}\right)\right) \times_{\mathcal{M}_{Y,+/-}(\mathfrak{t}, \eta)} \mathcal{M}_{X_{-}}\left(\mathfrak{s}_{-}\right) .
$$

Here $[u]\left(\mathcal{M}_{X_{+}}\left(\mathfrak{s}_{+}\right)\right) \times_{\mathcal{M}_{Y_{,+}-}(\mathfrak{t}, \eta)} \mathcal{M}_{X_{-}}\left(\mathfrak{s}_{-}\right)$is the fiber product of $\mathcal{M}_{X_{+}}\left(\mathfrak{s}_{+}\right)$and $\mathcal{M}_{X_{-}}\left(\mathfrak{s}_{-}\right)$over $\mathcal{M}_{Y,+/-}(\mathfrak{t}, \eta)$ with resepct to the maps $[u] \circ \pi_{+} \circ \partial_{\infty}^{+}$and $\pi_{-} \circ \partial_{\infty}^{-}$respectively.

Proof. We only give the proof for $[u]=0$ in $H^{1}(Y, \mathbb{Z}) /\left(\operatorname{Im}\left(i_{+}^{*}\right)+\operatorname{Im}\left(i_{-}^{*}\right)\right)$ here. Then $\mathfrak{s}=\mathfrak{s}_{+} \# \mathfrak{s}_{-}$is the $\operatorname{Spin}^{c}$ structure obtained by gluing the $\operatorname{Spin}^{c}$ structures $\mathfrak{s}_{ \pm}$long $Y$ using a gauge transformation which extends to either $X_{+}$or $X_{-}$. The proof for general $[u]$ is similar.

Given Theorem 4.9 and Proposition 4.4, we only need to prove that for a sufficiently large $R$, there exists an embedding

$$
\mathcal{M}_{X_{+}}\left(\mathfrak{s}_{+}\right) \times_{\mathcal{M}_{Y,+/-}(\mathfrak{t}, \eta)} \mathcal{M}_{X_{-}}\left(\mathfrak{s}_{-}\right) \longrightarrow \bigcup_{\mathfrak{s} \in \operatorname{Spin}^{c}\left(X, \mathfrak{s}_{ \pm}\right)} \mathcal{M}_{X(R)}(\mathfrak{s})
$$

The actual gluing procedure is fairly standard, see Theorem 4.9 in [20] (Cf. 29] and [12] in the instanton case) for the detailed gluing argument. Here we just set the stage in our case and leave the details to the reader.

Let $\left(\left[\mathbb{A}_{+}, \Psi_{+}\right],\left[\mathbb{A}_{-}, \Psi_{-}\right]\right) \in \mathcal{M}_{X_{+}}\left(\mathfrak{s}_{+}\right) \times_{\mathcal{M}_{Y,+/}(\mathfrak{t}, \eta)} \mathcal{M}_{X_{-}}\left(\mathfrak{s}_{-}\right)$, then we can choose two representatives, still denoted by $\left(\mathbb{A}_{+}, \Psi_{+}\right)$and $\left(\mathbb{A}_{-}, \Psi_{-}\right)$respectively. Then from Proposition 4.1 and Proposition 4.2, we know that $\left(\mathbb{A}_{ \pm}, \Psi_{ \pm}\right)$are solutions to the perturbed Seiberg-Witten equations (49) on $\left(X_{ \pm}(\infty), \mathfrak{s}_{ \pm}\right)$, decaying exponentially in the $C^{2}$-topology to $\left(A_{ \pm}, \psi_{ \pm}\right)$(solutions to the perturbed Seiberg-Witten equations (9) on $(Y, \mathfrak{t}))$ and that the cokernels of the operators $\mathcal{D}_{\left(\mathbb{A}_{ \pm}, \Psi_{ \pm}\right)}(53)$ are trivial.

As

$$
\pi_{+} \circ \partial_{\infty}^{+}\left(\left[\mathbb{A}_{+}, \Psi_{+}\right]\right)=\pi_{-} \circ \partial_{\infty}^{-}\left(\left[\mathbb{A}_{-}, \Psi_{-}\right]\right)
$$

there exists a gauge transformation in $C^{\infty}(Y, U(1))$ with $[g] \in \operatorname{Im}\left(i_{+}^{*}\right)+\operatorname{Im}\left(i_{-}^{*}\right)$ such that $\left(A_{+}, \psi_{+}\right)=g\left(A_{-}, \psi_{-}\right)$. As $[g] \in \operatorname{Im}\left(i_{+}^{*}\right)+\operatorname{Im}\left(i_{-}^{*}\right)$ then $g$ can be extended to either $X_{+}$or $X_{-}$.

Suppose that $[g] \in \operatorname{Im}\left(i_{+}^{*}\right)$. Let $g$ also represent the extended gauge transformation on $X_{+}(\infty)$. Apply the gluing argument to the element $\left(g^{-1}\left(\mathbb{A}_{+}, \Psi_{+}\right),\left(\mathbb{A}_{-}, \Psi_{-}\right)\right)$as in section 4.2 of [20]. Then for each sufficiently large $R$, there is a unique solution to the perturbed Seiberg-Witten equations (59) on $X(R)$ with some $\operatorname{Spin}^{c}$ structure in $\operatorname{Spin}^{c}\left(X, \mathfrak{s}_{ \pm}\right)$. Write $g^{-1}\left(\mathbb{A}_{+}, \Psi_{+}\right) \#_{R}\left(\mathbb{A}_{-}, \Psi_{-}\right)$for the resulting monopole on $X(R)$. If $[g] \in \operatorname{Im}\left(i_{-}^{*}\right)$, then the same procedure yields a unique monopole $\left(\mathbb{A}_{+}, \Psi_{+}\right) \#_{R} g\left(\mathbb{A}_{-}, \Psi_{-}\right)$. Notice that

$$
g^{-1}\left(\mathbb{A}_{+}, \Psi_{+}\right) \#_{R}\left(\mathbb{A}_{-}, \Psi_{-}\right)=\left(\mathbb{A}_{+}, \Psi_{+}\right) \#_{R} g\left(\mathbb{A}_{-}, \Psi_{-}\right)
$$


if and only if $[g] \in \operatorname{Im}\left(i_{+}^{*}\right) \cap \operatorname{Im}\left(i_{-}^{*}\right)$. This completes the sketch of the proof.

Notice that all the groups $\operatorname{Im}\left(i_{ \pm}^{*}\right), \operatorname{Im}\left(i_{+}^{*}\right) \cap \operatorname{Im}\left(i_{-}^{*}\right)$ and $\operatorname{Im}\left(i_{+}^{*}\right)+\operatorname{Im}\left(i_{-}^{*}\right)$ are subgroups of $\operatorname{Ker}\left(c_{1}(\mathfrak{t})\right)$, hence, there is an induced commutative diagram of $\mathbb{A}(Y)$-equivariant homomorphisms on the corresponding Seiberg-Witten-Floer homologies:

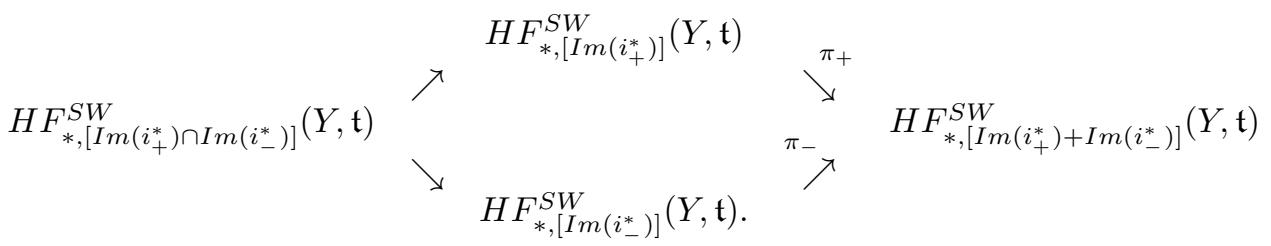

Recall that the relative invariants $S W_{X_{ \pm}}\left(\mathfrak{s}_{ \pm}, \cdot\right)$ take values in $H F_{*,\left[\operatorname{Im}\left(i_{+}^{*}\right)\right]}^{S W}(Y, \mathfrak{t})$ and $H F_{*,\left[I m\left(i_{-}^{*}\right)\right]}^{S W}(-Y,-\mathfrak{t})$ respectively. Under the homomorphism $\pi_{ \pm}$in $(63), \pi_{ \pm}\left(S W_{X_{ \pm}}\left(\mathfrak{s}_{ \pm}, \cdot\right)\right)$ take values in

$$
H F_{*,\left[\operatorname{Im}\left(i_{+}^{*}\right)+\operatorname{Im}\left(i_{-}^{*}\right)\right]}^{S W}(Y, \mathfrak{t}) \quad \text { and } \quad H F_{*,\left[\operatorname{Im}\left(i_{+}^{*}\right)+\operatorname{Im}\left(i_{-}^{*}\right)\right]}^{S W}(-Y,-\mathfrak{t})
$$

respectively.

From the definition of the relative invariants $S W_{X_{ \pm}}\left(s_{ \pm}, \cdot\right)$ and the definition of the SeibergWitten invariant for $(X(R), \mathfrak{s})$ with $\mathfrak{s}=\mathfrak{s}_{+} \#_{[u]} \mathfrak{s}_{-} \in \operatorname{Spin}^{c}\left(X, \mathfrak{s}_{ \pm}\right)$, Theorem 4.10 implies the following gluing formula.

Theorem 4.11. Suppose that $X$ is a closed 4-manifold with $b_{2}^{+} \geq 1$, which splits along a closed 3-manifold $(Y, \mathfrak{t})$ with $b_{1}(Y)>0$ and $c_{1}(\mathfrak{t})$ is non-torsion. Suppose that there are $\operatorname{Spin}^{c}$ structures $\mathfrak{s}_{+}$and $\mathfrak{s}_{-}$on $X_{+}$and $X_{-}$respectively such that $\left.\mathfrak{s}_{+}\right|_{Y}=\left.\mathfrak{s}_{-}\right|_{Y}=\mathfrak{t}$. Then for a $\operatorname{Spin}^{c}$ structure $\mathfrak{s}=\mathfrak{s}_{+} \#[u]^{\mathfrak{s}_{-}}$in $\operatorname{Spin}^{c}\left(X, \mathfrak{s}_{ \pm}\right)$, where $[u] \in H^{1}(Y, \mathbb{Z}) /\left(\operatorname{Im}\left(i_{+}^{*}\right)+\operatorname{Im}\left(i_{-}^{*}\right)\right)$, we have the following gluing formula for the Seiberg-Witten invariants:

$$
S W_{X}\left(\mathfrak{s}, z_{+} z_{-}\right)=\left\langle[u]\left(\pi_{+}\left(S W_{X_{+}}\left(\mathfrak{s}_{+}, z_{+}\right)\right)\right), \pi_{-}\left(S W_{X_{-}}\left(\mathfrak{s}_{-}, z_{-}\right)\right)\right\rangle
$$

where $[u]$ acts on $H F_{*,\left[\operatorname{Im}\left(i_{+}^{*}\right)+\operatorname{Im}\left(i_{-}^{*}\right)\right]}^{S W}(-Y,-\mathfrak{t})$ as in Remark 3.27, $\pi_{ \pm}$are the induced $\mathbb{A}(Y)$ equivariant homomorphisms from $\operatorname{Im}\left(i_{ \pm}^{*}\right) \subseteq \operatorname{Im}\left(i_{+}^{*}\right)+\operatorname{Im}\left(i_{-}^{*}\right)$ (see Diagram (63)), and $\langle$,$\rangle is$ the pairing on

$$
H F_{*,\left[\operatorname{Im}\left(i_{+}^{*}\right)+\operatorname{Im}\left(i_{-}^{*}\right)\right]}^{S W}(Y, \mathfrak{t}) \times H F_{*,\left[\operatorname{Im}\left(i_{+}^{*}\right)+\operatorname{Im}\left(i_{-}^{*}\right)\right]}^{S W}(-Y,-\mathfrak{t}),
$$

with the degrees in $H F_{*,\left[\operatorname{Im}\left(i_{+}^{*}\right)+\operatorname{Im}\left(i_{-}^{*}\right)\right]}(-Y,-\mathfrak{t})$ shifted by

$$
d_{X}(\mathfrak{s})=\frac{1}{4}\left(c_{1}(\mathfrak{s})^{2}-(2 \chi(X)+3 \sigma(X))\right)=\operatorname{deg}\left(z_{1}\right)+\operatorname{deg}\left(z_{2}\right),
$$

and $z_{ \pm} \in \mathbb{A}\left(X_{ \pm}\right)$. When $b_{2}^{+}=1$, the Seiberg-Witten invariants $S W_{X}(\mathfrak{s}, \cdot)$ is defined with a fixed orientation on $H^{2,+}(X, \mathbb{R})$ such that $c_{1}(\mathfrak{s}) \cdot \omega^{+}>0$ for an oriented generator $\omega^{+}$of $H^{2,+}(X, \mathbb{R})$. 
Note that $S W_{X}\left(\mathfrak{s}, z_{1} z_{2}\right)=0$ for any $\mathfrak{s}$ with $d_{X}(\mathfrak{s}) \neq \operatorname{deg}\left(z_{1}\right)+\operatorname{deg}\left(z_{2}\right)$, and

$$
\mathcal{S}=\left\{\mathfrak{s} \in \operatorname{Spin}^{c}\left(X, \mathfrak{s}_{ \pm}\right): d_{X}(\mathfrak{s})=\operatorname{deg}\left(z_{1}\right)+\operatorname{deg}\left(z_{2}\right)\right\}
$$

is an affine space over $\frac{\operatorname{Ker}\left(c_{1}(\mathfrak{t})\right)}{\operatorname{Im}\left(i_{+}^{*}\right)+\operatorname{Im}\left(i_{-}^{*}\right)}$. Summing the gluing formulae (64) over all $\operatorname{Spin}^{c}$ structures in $\operatorname{Spin}^{c}\left(X, \mathfrak{s}_{ \pm}\right)$, we obtain that

$$
\sum_{\mathfrak{s} \in \mathcal{S}} S W_{X}\left(\mathfrak{s}, z_{+} z_{-}\right)=\left\langle\left(\pi\left(S W_{X_{+}}\left(\mathfrak{s}_{+}, z_{+}\right)\right), \pi\left(S W_{X_{-}}\left(\mathfrak{s}_{-}, z_{-}\right)\right)\right\rangle .\right.
$$

Here $\pi$ is the map (Cf. (45) ) from $H F_{*,\left[\operatorname{Im}\left(i_{ \pm}^{*}\right)\right]}^{S W}( \pm Y, \pm \eta)$ to

$$
H F_{*,\left[K e r\left(c_{1}(\eta)\right)\right]}^{S W}( \pm Y, \pm \eta) \cong H F_{*}^{S W}( \pm Y, \pm \eta)
$$

under the periodicity map (43), $\pi\left(S W_{X_{ \pm}}\left(\mathfrak{s}_{ \pm}, z_{ \pm}\right)\right)$take values in $H F_{*}^{S W}( \pm Y, \pm \mathfrak{t})$, and the right hand side of (65) the natural pairing on

$$
H F_{*}^{S W}(Y, \mathfrak{t}) \times H F_{*}^{S W}(-Y,-\mathfrak{t}) .
$$

In particular, in the case of $\operatorname{Im}\left(i_{+}^{*}\right)+\operatorname{Im}\left(i_{-}^{*}\right)=\operatorname{Ker}\left(c_{1}(\mathfrak{t})\right)$, the left hand side of (65) has at most one term.

As an application of these gluing formulae, we briefly mention some results in [26]. Applying the gluing theorem, Muñoz and Wang obtained a ring structure on $H F_{*}^{S W}\left(\Sigma_{g} \times S^{1}, \mathfrak{s}_{r}\right)$ where $\Sigma_{g}$ is a closed surface of genus $g \geq 1$ and $\mathfrak{s}_{r}$ is the $\operatorname{Spin}^{c}$ structure on $\Sigma_{g} \times S^{1}$ with $c_{1}\left(\mathfrak{s}_{r}\right)=2 r P D\left(S^{1}\right)$ $(-(g-1) \leq r \leq g-1)$. Note that $\operatorname{Ker}\left(c_{1}\left(\mathfrak{s}_{r}\right)\right)=H^{1}\left(\Sigma_{g}, \mathbb{Z}\right)$ and $H^{1}\left(\Sigma_{g} \times S^{1}, \mathbb{Z}\right) / \operatorname{Ker}\left(c_{1}\left(\mathfrak{s}_{r}\right)\right) \cong \mathbb{Z}$. Set $d=g-1-|r|$, we only discuss the case of $r \neq 0$ here. Denote by $\mathfrak{s}_{r}$ also the $\operatorname{Spin}^{c}$ structure $^{2}$ on $\Sigma_{g} \times D^{2}$ with $c_{1}\left(\mathfrak{s}_{r}\right)=2 r P D\left(\left[p t \times D^{2}\right]\right)$, where $D^{2}$ is a 2 -dimensional disc. The relative invariants for $\Sigma_{g} \times D^{2}$ define a map

$$
\begin{aligned}
\mathbb{A}\left(\Sigma_{g} \times D^{2}\right) & =\mathbb{A}\left(\Sigma_{g}\right) \longrightarrow H F_{*}^{S W}\left(\Sigma_{g} \times S^{1}, \mathfrak{s}_{r}\right) \\
z & \mapsto S W_{\Sigma_{g} \times D^{2}}\left(\mathfrak{s}_{r}, z\right) .
\end{aligned}
$$

Then the gluing theorem (Theorem 4.11) tells us that

$$
\left\langle S W_{\Sigma_{g} \times D^{2}}\left(\mathfrak{s}_{r}, z_{1}\right), S W_{\Sigma_{g} \times D^{2}}\left(\mathfrak{s}_{r}, z_{2}\right)\right\rangle=\sum_{n \in \mathbb{Z}} S W_{\Sigma_{g} \times S^{2}}\left(\mathfrak{s}_{r}+n P D\left(\Sigma_{g}\right), z_{1} z_{2}\right),
$$

for any $z_{1}, z_{2} \in \mathbb{A}\left(\Sigma_{g}\right)$. For dimensional reasons there is at most one $n \in \mathbb{Z}$ such that $S W_{\Sigma_{g} \times S^{2}}\left(\mathfrak{s}_{r}+2 n P D\left(\left[\Sigma_{g}\right]\right), z_{1} z_{2}\right)$ is non-zero. If $\operatorname{deg}\left(z_{1}\right)+\operatorname{deg}\left(z_{2}\right)=2 d$, then

$$
\left\langle S W_{\Sigma_{g} \times D^{2}}\left(\mathfrak{s}_{r}, z_{1}\right), S W_{\Sigma_{g} \times D^{2}}\left(\mathfrak{s}_{r}, z_{2}\right)\right\rangle=\left\langle z_{1} z_{2},\left[\operatorname{Sym}^{d}\left(\Sigma_{g}\right)\right]\right\rangle .
$$

These pairing, along with others in [26], can be used to study the structure of $H F_{*}^{S W}\left(\Sigma_{g} \times S^{1}, \mathfrak{s}_{r}\right)$, see 26] for explicit calculations. 


\section{References}

[1] M. Atiyah, V. Patodi, I. Singer, Spectral asymmetry and Riemannian geometry. I. II. III., Math. Proc. Cambridge Philos. Soc. 77 (1775a) 43-69, 78 (175b) 405-432, 79 (1976) 71-99;

[2] D. Auckly, Surgery, knots and the Seiberg-Witten equations, Lectures for the 1995 TGRCIW,

[3] B. Booss-Bavnbek, M. Marcolli, B. L. Wang Weak UCP and perturbed monopole equation, preprint;

[4] A.L. Carey, J. McCarthy, B.L. Wang, R.B. Zhang, Seiberg-Witten Monopoles in Three Dimensions, Lett. in Math. Phys., Vol. 39, 213-228, 1997;

[5] A. L. Carey, M. Marcolli, B. L. Wang, Exact triangles for Seiberg-Witten-Floer theory. Part I: the geometric triangle, preprint;

[6] W. Chen, Casson's invariant and Seiberg-Witten gauge theory, preprint;

[7] S.K. Donaldson, The Seiberg-Witten equations and 4-manifold topology, Bull. AMS, Vol.33 N.1 (1996), 45-70;

[8] A. Floer, An instanton-invariant for 3-manifolds, Comm. Math. Phys. 118 (1988), 215240 ;

[9] A. Floer, The unregularized gradient flow of the symplectic action. Comm. Pure Appl. Math. 41 (1988), no. 6, 775-813;

[10] D. Freed, K. Uhlenbeck, Instantons and four-manifolds, MSRI Publications, 1. SpringerVerlag, New York-Berlin, 1984;

[11] K.A. Froyshov, The Seiberg-Witten equations and four-manifolds with boundary, Math. Res. Lett. 3 (1996), N.3, 373-390;

[12] K Fukaya, Instanton homology for oriented 3-manifolds, Adv. Studies in Pure Math. Vol. 20 1992, 1-92;

[13] J. Jost, X. Ping, G. Wang, Variational aspects of the Seiberg-Witten functional. Calc. Var. Partial Diff. Equ. 4 (1996), no. 3, 205-218

[14] P.B. Kronheimer, Embedded surfaces and gauge theory in three and four dimensions, Surveys in differential geometry, Vol. III (Cambridge, MA, 1996), 243-298, Int. Press, Boston, MA, 1998.

[15] P.B. Kronheimer, T.S. Mrowka, The Genus of Embedded Surfaces in the Projective Plane, Math. Research Lett. 1 (1994), 797-808; 
[16] P.B.Kronheimer, T.S.Mrowka, Monopoles and contact structures, preprint;

[17] Y. Lim, Seiberg-Witten invariants for three-manifolds and product formulae, preprint;

[18] R.B. Lockhard, R.C. McOwen, Elliptic operators on non-compact manifolds, Ann. Sci. Norm. Sup. Pisa, IV-12 (1985), 409-446;

[19] M. Marcolli, Seiberg-Witten-Floer Homology and Heegard Splittings Intern. Jour. of Maths., Vol 7, No. 5 (1996) 671-696; see also dg-ga/9601011;

[20] M. Marcolli, B. L. Wang Equivariant Seiberg-Witten-Floer theory, preprint;

[21] M. Marcolli, B. L. Wang Exact triangles in Seiberg-Witten-Floer theory. Part IV: $\mathbb{Z}$ graded monopole homology, preprint;

[22] G. Meng, C. H. Taubes $\underline{S W}=$ Milnor Torsion, Harvard University, preprint;

[23] J. W. Morgan, The Seiberg-Witten equations and applications to the topology of smooth four-manifolds, Princeton Univ. press, 1996;

[24] J.W. Morgan, T.S. Mrowka and D. Ruberman, The $L^{2}$-moduli space and a vanishing theorem for Donaldson polynomial Invariants, Monographs in Geometry and Topology, Vol 2, 1994.

[25] J. W. Morgan, Z. Szabo and C. H. Taubes, A product formula for the Seiberg-Witten invariants and the generalized Thom Conjecture, J. Differential Geom. 44 (1996), no. 4, 706-788.;

[26] V. Muñoz, B. L. Wang, Seiberg-Witten-Floer homology of a surface times a circle, preprint;

[27] L. Nicolaescu, Notes on Seiberg-Witten theory, American Mathematical Society, 2000.

[28] P. Ozsvath, Z. Szabo, Holomorphic disks and three-manifold invariants: properties and applications, preprint, math.SG/0105202;

[29] C. H. Taubes, Gauge theory on symptotically periodic 4-manifolds, J. Differential Geom. 25 (1987), no. 3, 363-430;

[30] C. H. Taubes, Casson's invariants and gauge theory, Journ. of Diff. Geom. 31 (1990) 547-599;

[31] C. H. Taubes, $S W \Rightarrow G r$, From the Seiberg-Witten equations to pseudo-holomorphic curves, Jour, Amer. Math. Soc. Vol 9, (1996), 845-918.

[32] C. H. Taubes, $L^{2}$ moduli spaces on 4-manifolds with cylindrical ends, Monographs in Geometry and Topology, Vol. 1, International Press, 1993.

[33] C.H. Taubes, The Seiberg-Witten invariants and 4-manifolds with essential tori, Geom. Topol. 5 (2001), 441-519. 
[34] C.H. Taubes, Seiberg-Witten and Gromov invariants for symplectic 4-manifolds, International Press, 2000.

[35] M. E. Taylor, Partial differential equations III, Nonlinear equations, Springer-Verlag, 1997.

[36] V. G. Turaev, Torsion invariants of $\operatorname{Spin}^{c}$ structures on 3-manifolds, Math. Res. Letters 4 (1997) 679-695.

[37] B. L Wang, Seiberg-Witten monopoles on three-manifolds Ph.D. thesis, (Adelaide) 1997;

[38] E. Witten, Monopoles and four-manifolds, Math. Research Lett. 1 (1994) 769-796;

\section{A.L. Carey,}

School of Mathematical Sciences, Australian National University, Canberra ACT, Australia acarey@maths.anu.edu.au

\section{B.L. Wang,}

Department of Pure Mathematics, University of Adelaide, Adelaide SA 5005

bwang@maths.adelaide.edu.au

Max-Planck-Institut für Mathematik, D-53111 Bonn, Germany

bwang@mpim-bonn.mpg.de 